\title{
Effect of the CPAP-SAVER Intervention on Adherence Among Adults with Newly Diagnosed Obstructive Sleep Apnea
}

April L. Shapiro

Follow this and additional works at: https://researchrepository.wvu.edu/etd

\section{Recommended Citation}

Shapiro, April L., "Effect of the CPAP-SAVER Intervention on Adherence Among Adults with Newly Diagnosed Obstructive Sleep Apnea" (2017). Graduate Theses, Dissertations, and Problem Reports. 6617. https://researchrepository.wvu.edu/etd/6617

This Dissertation is protected by copyright and/or related rights. It has been brought to you by the The Research Repository @ WVU with permission from the rights-holder(s). You are free to use this Dissertation in any way that is permitted by the copyright and related rights legislation that applies to your use. For other uses you must obtain permission from the rights-holder(s) directly, unless additional rights are indicated by a Creative Commons license in the record and/ or on the work itself. This Dissertation has been accepted for inclusion in WVU Graduate Theses, Dissertations, and Problem Reports collection by an authorized administrator of The Research Repository @ WVU.

For more information, please contact researchrepository@mail.wvu.edu. 
Effect of the CPAP-SAVER Intervention on Adherence Among

Adults with Newly Diagnosed Obstructive Sleep Apnea

\author{
April L. Shapiro \\ Dissertation submitted \\ to the School of Nursing \\ at West Virginia University \\ in partial fulfillment of the requirements for the degree of \\ Doctor of Philosophy in \\ Nursing
}

Susan McCrone, PhD, RN, Chair

Gina Maiocco, PhD, RN

Mary Jane Smith, PhD, RN

Hawley Montgomery-Downs, PhD

Jamie DeCoster, PhD

Department of Nursing

\author{
Morgantown, West Virginia \\ 2017
}

Keywords: CPAP adherence, obstructive sleep apnea, nursing intervention, Theory of Planned Behavior

Copyright 2017 April L. Shapiro 


\title{
ABSTRACT \\ Effect of the CPAP-SAVER Intervention on Adherence Among
}

\section{Adults with Newly Diagnosed Obstructive Sleep Apnea}

\begin{abstract}
April L. Shapiro
Introduction: Obstructive sleep apnea (OSA) affects 25 million adults in the United States. Continuous positive airway pressure (CPAP) is the treatment of choice, but adherence is poor. Many previous CPAP adherence interventions were not theory based, tended to impose time and cost burden, and did not focus on OSA airway-brain mechanism education or OSA-CPAP performance feedback. A new, multidimensional intervention, known as CPAP-SAVER, was developed, based on the Theory of Planned Behavior (TPB), knowledge of CPAP facilitators and barriers, characteristics of CPAP adherers and nonadherers, and behavior change techniques. The purpose of this study was to examine the effect of the CPAP-SAVER intervention on adherence among adults with newly diagnosed OSA. Additional aims were to examine the effect of the intervention on anxiety, apnea beliefs, attitude, subjective norm, perceived behavioral control, and intention; predictors of intention and behavior were also determined.

Method: After IRB approval and consent, 66 participants from two home medical supply facilities were recruited over ten months for the experimental study. Participants were randomly assigned to intervention or standard care groups. Standard care included CPAP teaching and follow-up. The intervention involved support calls; the use of an airway model, video, education sheet, and report card; and standard care. Data were collected using a demographic survey, a TPB Questionnaire, the Apnea Beliefs Scale, the Beck Anxiety Inventory, the sleep study report, and the CPAP modem. Data were analyzed using SPSS 24, with alpha set at .05. Assumptions testing, scale reliability testing, frequencies, and descriptives were analyzed. Statistical analyses to answer the research questions included a chi-square test of independence, mixed betweenwithin subjects ANOVAs, t-tests, and multiple and logistic regressions.

Results: There was no significant effect of the intervention on CPAP adherence at one month. Anxiety significantly decreased over time. Beliefs were higher at one month in the intervention group compared to standard care; there were no significant differences in attitude, subjective norm, or perceived behavioral control in the groups over time. CPAP adherence attitude, subjective norm, and perceived behavioral control significantly predicted CPAP adherence intention and explained $52.1 \%$ of the total variance; each of the variables demonstrated a significant, unique contribution to the variance in CPAP adherence intention. CPAP adherence intention significantly explained $14.1 \%$ to $21.0 \%$ of the variance in CPAP adherence behavior. Most intervention group participants rated the CPAP-SAVER intervention components as 3 or 4 (somewhat or extremely helpful, liked, understood, and motivating) on a Likert scale of 0 to 4. Conclusion and Implications: The CPAP-SAVER study yielded mixed results, however, the intervention may provide groundwork for the eventual development of a clinical guideline for OSA-CPAP management to benefit both patients and practitioners. Replicating the CPAPSAVER study in a larger, more diverse population and synthesizing the results with seminal works are the recommended next steps in translating this research into policy and practice.
\end{abstract}




\section{Dedication}

My husband, Steve: You were my rock throughout this journey; you figuratively and literally took this journey with me, supporting me and listening when I had good days and bad, sometimes both at once; driving to "Motown" (as we called it) and Romney; or going to research conferences in DC, Florida, and Indiana. We did this together and I am forever grateful for your love, support, and sacrifice. I love you dearly.

My daughter, Sierra: My life forever changed when you came into this world. You are the reason I do what I do and the reason I became a nurse in the first place. I always strive to be a good role model for you and the best mother I can be to you. I know you were worried when I started this program - that I was taking on too much - but, regardless, you supported and encouraged me every step of the way. You listened to all my concerns without judgement and helped me stay optimistic when things were stressful. I loved all your texts, especially the ones with funny YouTube videos, what was happening in politics, or new songs. You kept me grounded and centered. I love you more than words can express, Sierra.

My parents (my mom, Clara, and my dad, Lloyd, and his wife, Carol): Thank you for encouraging me every step of the way. Mom, I know you were worried the program would be too much, but you were always supportive and eager to show pictures of my accomplishments to family and friends. Dad, you and Carol would always call and ask how the study was going and how many participants I had, and I was always so excited to give you a progress report. As my parents, your confidence and pride in me are so rewarding and encouraging. Thanks for loving me and supporting me along every step of life... I love you more than words can express.

My sister, Kelli: Thanks for all the Sunday meals and leftovers, texts just to check in, reminders to rest and relax a little, camping trips to unwind and regroup, laughs until we cried, 
and for just being the awesome sister you are. God blessed me when he gave me you as my sister. Love you dearly, Kel.

My nephew, Josh: Thanks for just being you and making me laugh a lot. I cherish all of our time together, especially the fun and silly arguments we have playing video games, basketball, and baseball... you keep me young, grounded, and destressed. Auntie April loves ya, pal.

Dr. Susan McCrone: Dr. McCrone, thank you for being my utmost source of encouragement and support in seeing this study through to fruition. Thank you for building my confidence throughout every step of this journey, but most for encouraging me to take the first step. It was your careful guidance and constant encouragement that led me to implement my intervention. I cannot thank you enough for your mentorship. 


\section{Acknowledgements}

WVU School of Nursing

Dissertation Committee - Dr. Susan McCrone (Chair), Dr. Gina Maiocco, Dr. Mary Jane Smith, Dr. Hawley Montgomery-Downs, and Dr. Jamie DeCoster: Thank you for your unrelenting guidance, support, and patience throughout the dissertation process. Dean Tara Hulsey: A special thank you for all of your support, encouragement, and generous funding to implement my dissertation.

Dr. Gina Maiocco, PhD Program Director: Thank you for the guidance, support, encouragement, and generous funding. You were a wonderful role model to me, as both a leader and a researcher.

Dr. Mary Jane Smith: Meeting you at the PhD Program Open House on Wednesday, January 5, 2011, changed my life forever. I am eternally grateful to you.

PhD Faculty: There is a piece of each and every one of you in my dissertation; thank you for inspiring me, expanding my mind, and being exemplary teachers and role models.

PhD Classmates - Karen Jagiello, MS, RNC, and Deborah Strickland, MS, RN:

Thank you for listening, caring, inspiring, and supporting. You are dear to me. School of Nursing Offices: Student Services, Financial Aid, Business, and Nursing Research: Thanks for your help with tuition waivers and scholarships, processing my dissertation funds, and making lots and lots of copies. You kept my wheels in motion. E. Jane Martin and the Anna Mary Miller Family: Thank you tremendously for the scholarship funding. I hope I would have made Anna Mary Miller proud.

WVU Statistics Department

Dr. Stacey Culp: Thank you for teaching me so much about statistics and SPSS; I would 
not have been able to complete the analyses in this study without the strong foundation of knowledge you helped build.

West Virginia Center for Nursing

Duane Napier, Christopher Ross, and Drema Pierson: Before I came into this program, my excuses were, "I don't have the time and I definitely don't have the money." You took away my biggest excuse. You made sure I had the money, through scholarship funding, to make my dream a reality. I will never be able to thank you enough.

Home Medical Supply Facilities

My Research Assistants: Without you ladies, I could not have implemented this study. I know I have thanked you over and over, but I will say it again: Thank you for your dedication, patience, and persistence. We got our 66!

Study Participants: This dissertation study would not have been possible without each of you. Thank you for helping to advance nursing science and my understanding of CPAP adherence.

Mineral County Schools

Rita Jean Harber: Thank you for your support and encouragement. I will never forget the confidence you instilled in me as I struggled with the decision of whether to apply to the $\mathrm{PhD}$ program or not. You are a wonderful mentor and friend to me.

Jennifer Simpson, Amy DelSignore, and Petula Staggs: Thank you for your patience, support, and encouragement. I appreciate each and every sacrifice you made for me to be able to realize my dream. I did it, girls!

Shawn Dilly and Scott Staley: Thank you for your understanding and encouragement throughout these last six years; I could not have accomplished this without your support. 
Nursing students: Dear students, I continue to learn from you and for you every day.

You keep me young and thirsty for knowledge. Thank you for just being you. Allegany College of Maryland

Dr. Geraldine Domashinski and Barbara Williams: Even though you both have passed from this world, you are with me in spirit. From the day I met you, you inspired me to climb the nursing ladder and reach for the stars. I hope I made you proud. 


\section{List of Tables}

Table 1 Typologies of CPAP Adherers and Nonadherers Considered in the CPAP-SAVER Intervention Development

Table 2 Abraham and Michie's (2008) Behavior Change Techniques Operationalized in the CPAP-SAVER Intervention Study

Table 3 Definition, Operationalization, and Measurement of Each Variable in the CPAPSAVER Intervention Study

Table 4 Direct Questions for Measuring CPAP Adherence Attitude, CPAP Adherence Subjective Norm, CPAP Adherence Perceived Behavioral Control, and CPAP Adherence Intention in the CPAP-SAVER Intervention Study

Table $5 \quad$ CPAP-SAVER Intervention Study Protocol

Table 6 Demographics of Participants in the CPAP-SAVER Intervention Study $(N=66)$

Table 7 Sleep and OSA-Related Demographics of Participants in the CPAP-SAVER Intervention Study $(N=66)$

Table 8 CPAP-Related Characteristics of Participants in the CPAP-SAVER Intervention Study $(N=66)$

Table 9 OSA- and CPAP-Related Descriptives for Participants in the CPAP-SAVER Intervention Study $(N=66)$

Table 10 Demographics of Participants in the CPAP-SAVER Intervention Study by Group

Table 11 Sleep and OSA-Related Demographics of Participants in the CPAP-SAVER Intervention Study by Group

Table 12 CPAP-Related Characteristics of Participants in the CPAP-SAVER Intervention Study by Group

Table 13 OSA- and CPAP-Related Descriptives for Participants in the CPAP-SAVER Intervention Study by Group

Table 14 Mean Instrument Scores for Participants in the CPAP-SAVER Intervention Study by Group

Table 15 OSA- and CPAP-Related Descriptives for Participants in the CPAP-SAVER Intervention Study by Gender

Table 16 Mean Instrument Scores for Participants in the CPAP-SAVER Intervention Study by Gender

Table 17 Frequencies of Report Card Grades for Intervention Group Participants in the CPAP-SAVER Study

Table 18 Intervention Effectiveness Survey Results: Mean Scores for the CPAP-SAVER Study Components 


\section{List of Figures}

Figure 1 Theory of Planned Behavior

Figure 2 CPAP-SAVER Intervention Using the Theory of Planned Behavior as the Guiding Framework

Figure 3 A Priori Power Analysis Using G*Power 3.1 


\section{List of Appendices}

Appendix A Demographics Survey and Instruments

Appendix B Support/Subjective Norm Call Log

Appendix C Education Sheet

Appendix D CPAP Report Card

Appendix E CPAP-SAVER Intervention OSA-CPAP Data Log

Appendix F CPAP-SAVER Intervention Fidelity Checklist

Appendix G CPAP-SAVER Intervention Effectiveness Survey

Appendix H Incidental Findings Letter

Appendix I Confidentiality Agreement 


\section{List of Abbreviations}

AASM

ABS

ACTI

AHI

BAI

BMI

CBT

CITI

CPAP

CPAP-SAVER

ED

EDS

HAPA

HBM

MET

MINT

NFM

OSA

PFM

PMR

PSG

RDI

RERA

RT

SASMP

SCT

SMC

TLC-CPAP

TPB
American Academy of Sleep Medicine

Apnea Beliefs Scale

Attitudes to CPAP Inventory

Apnea-Hypopnea Index

Beck Anxiety Inventory

Body Mass Index

Cognitive Behavioral Theory

Collaborative Institutional Training Initiative

Continuous Positive Airway Pressure

Continuous Positive Airway Pressure-Support/Subjective Norm Calls,

Airway Model, Video, Education Sheet, Report Card

Education

Excessive Daytime Sleepiness

Health Action Process Approach

Health Belief Model

Motivational Enhancement Theory

Motivational Interview Nurse Therapy

Negatively-Framed Messaging

Obstructive Sleep Apnea

Positively-Framed Messaging

Progressive Muscle Relaxation

Polysomnogram or Polysomnographer

Respiratory Disturbance Index

Respiratory Effort-Related Arousals

Respiratory Therapist(s)

Sleep-Apnea Self-Management Program

Social Cognitive Theory

Stage-Matched Care

Telephone Linked Communication-Continuous Positive Airway Pressure Theory of Planned Behavior 


\section{Table of Contents}

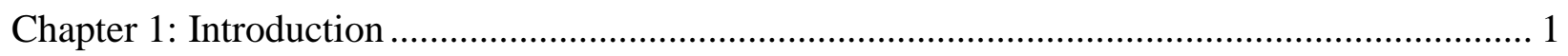

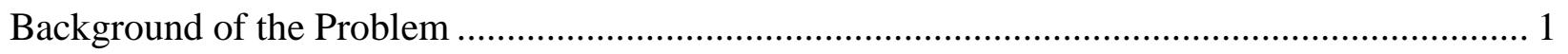

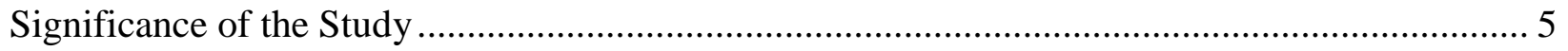

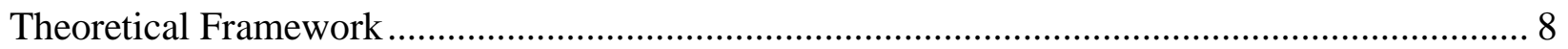

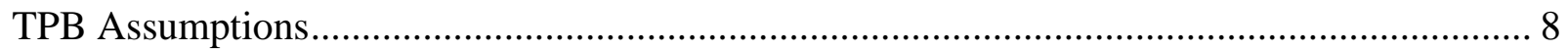

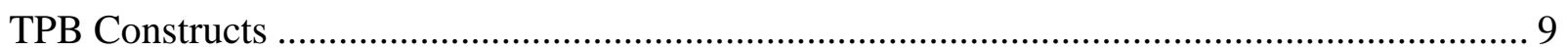

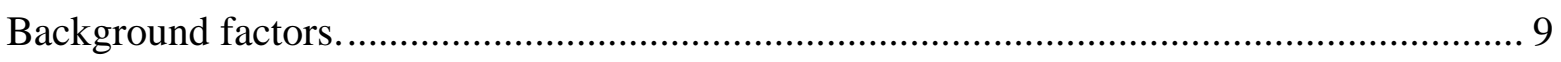

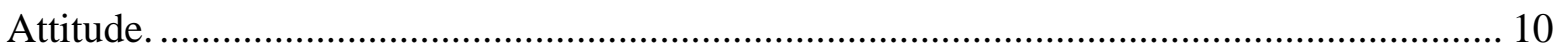

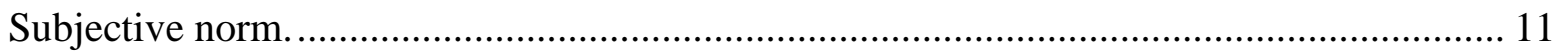

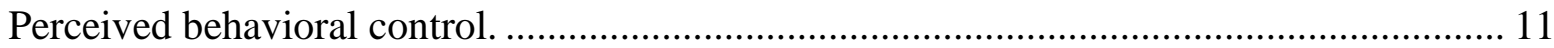

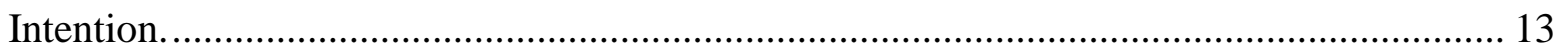

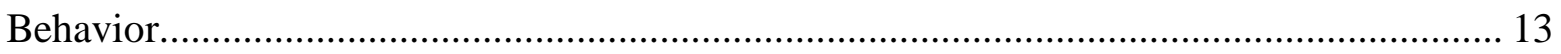

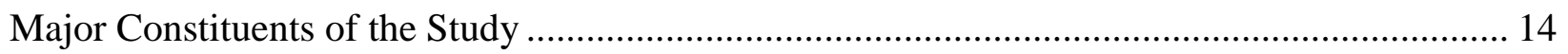

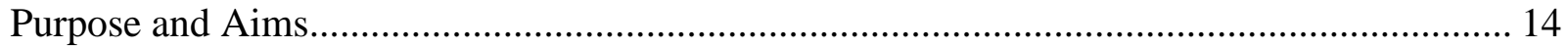

Research Questions and Hypotheses .............................................................................. 15

Definitions, Operationalization, and Measures for Variables ..................................................... 16

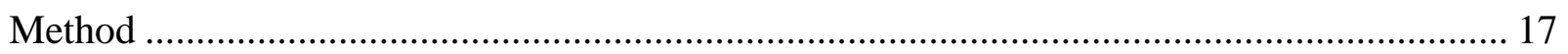

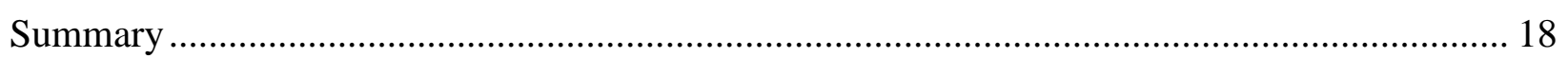

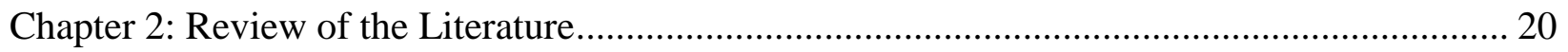

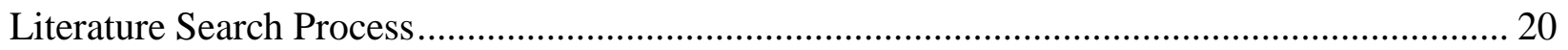

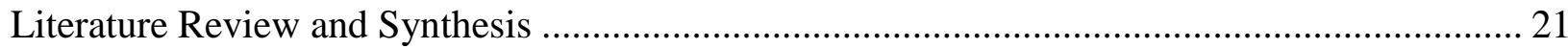

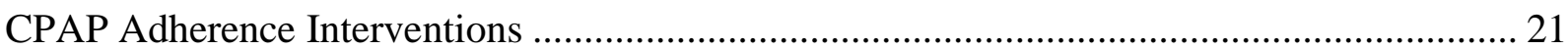

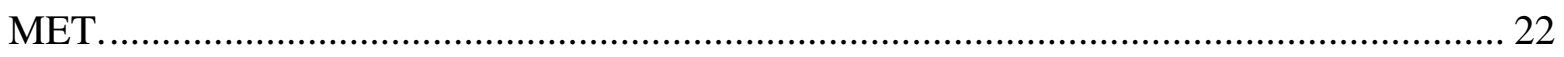

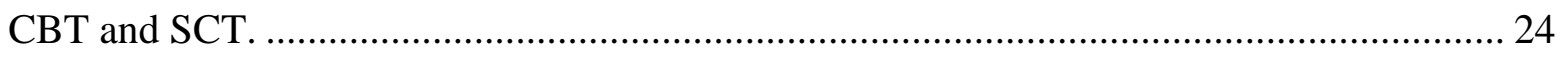

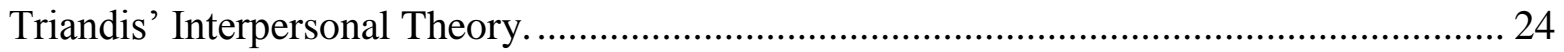

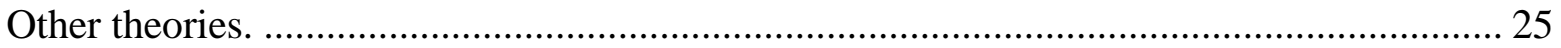

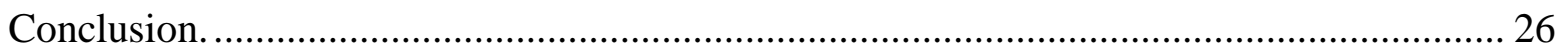

TPB-Based Health-Related Interventions ......................................................................... 26

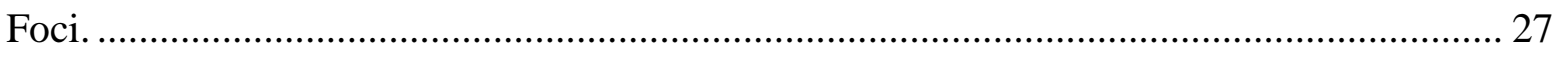

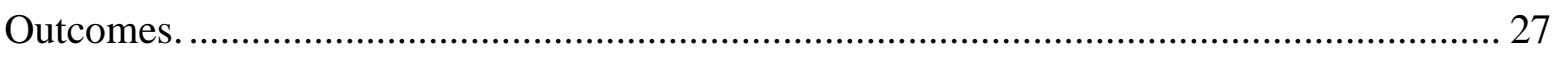

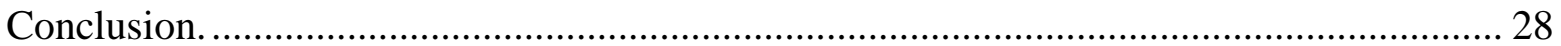


Measurement of the TPB Constructs in OSA-CPAP Samples ........................................... 28

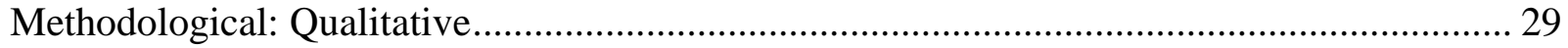

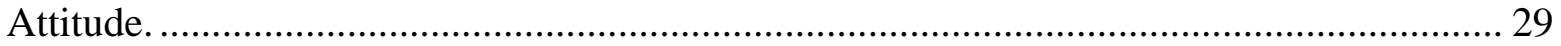

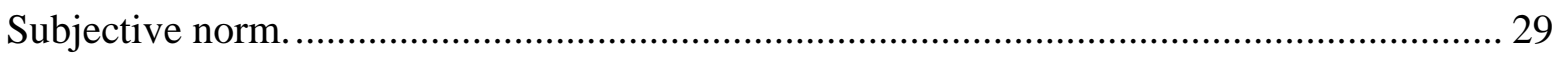

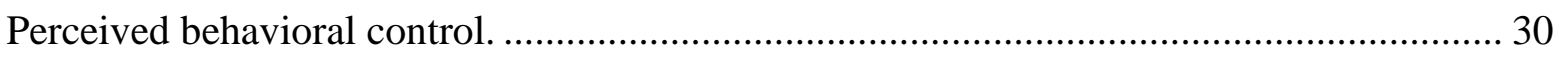

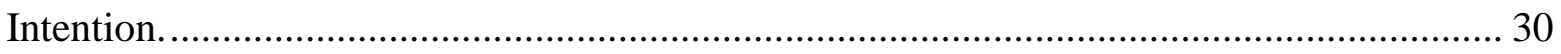

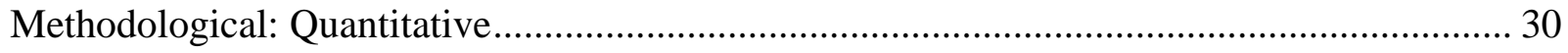

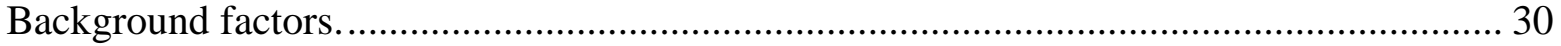

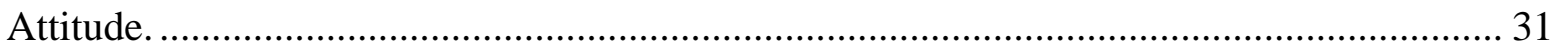

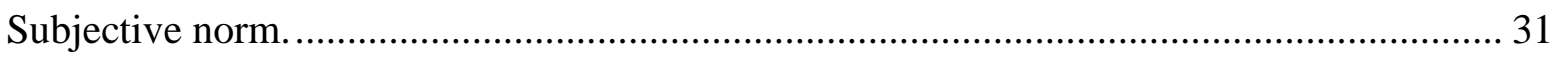

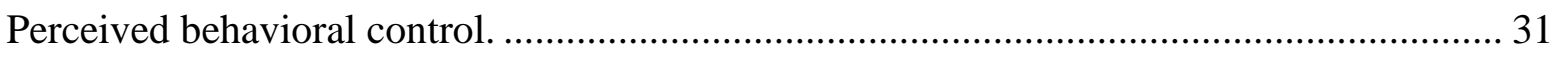

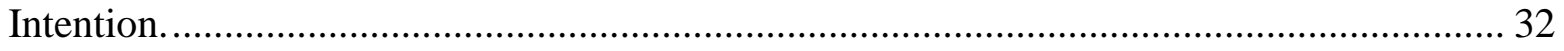

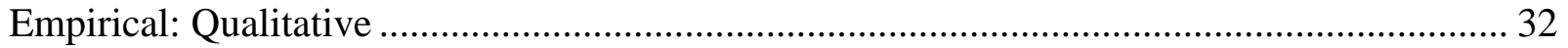

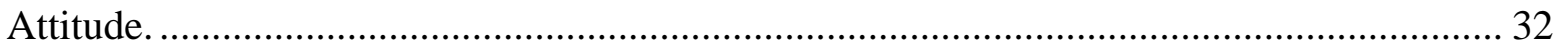

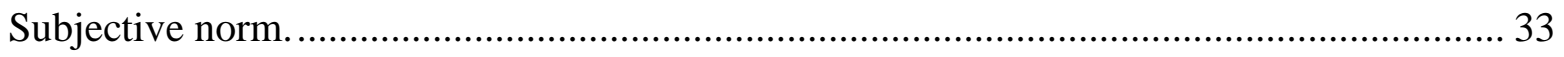

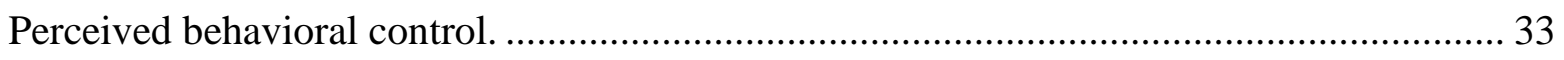

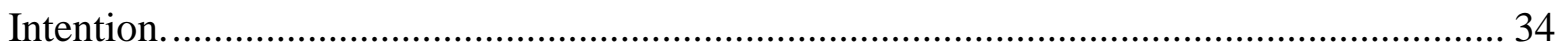

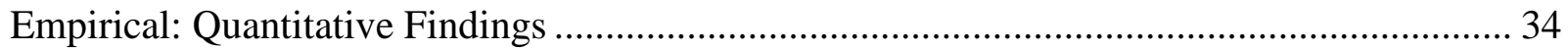

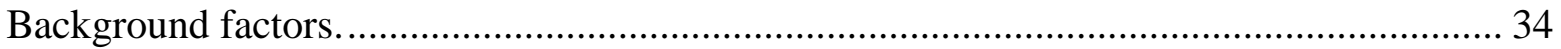

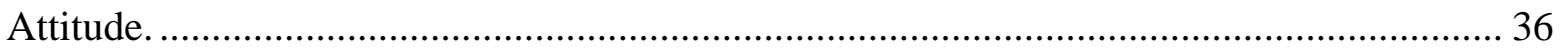

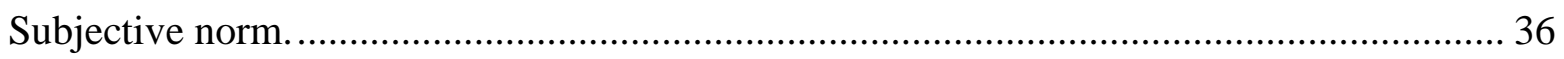

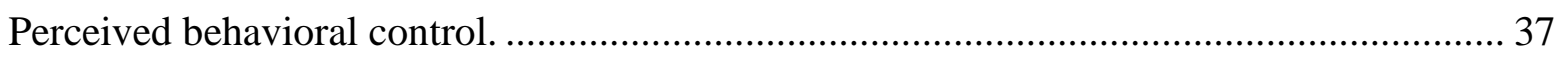

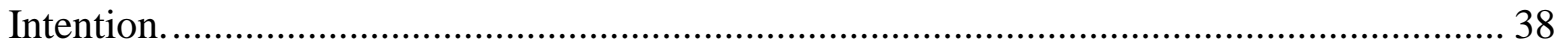

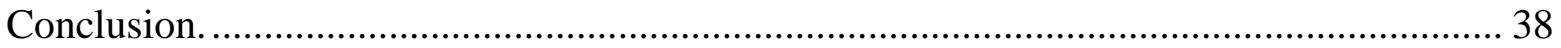

The CPAP-SAVER Intervention and Its Components .................................................... 39

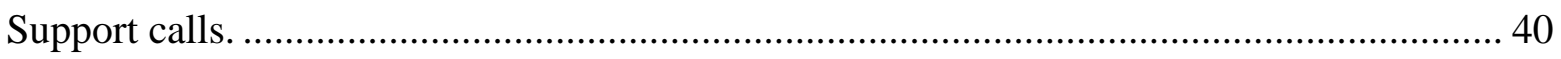

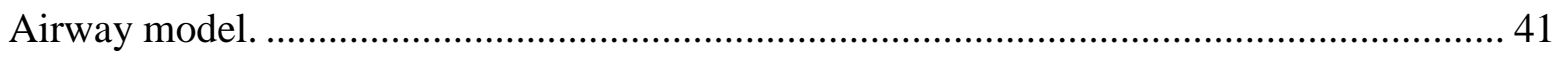

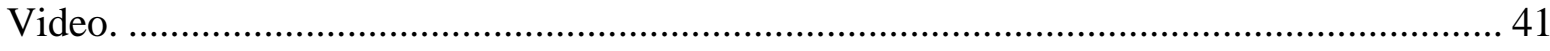

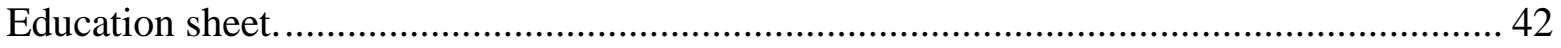

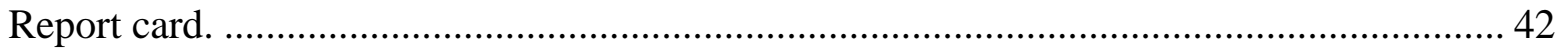

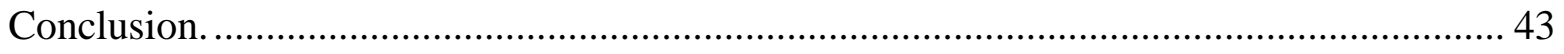

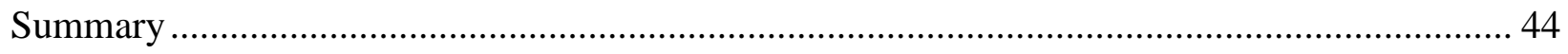




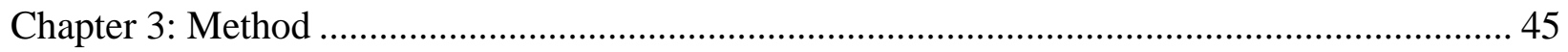

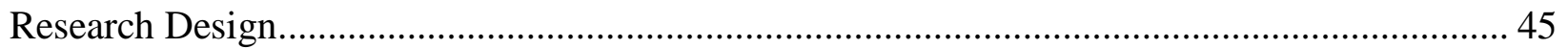

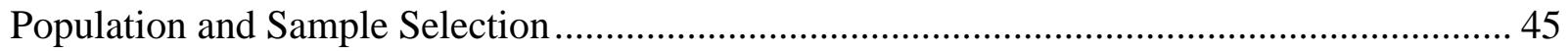

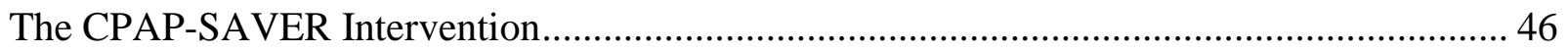

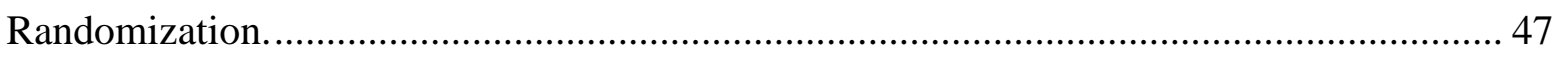

Intervention components, dosing, and frequency............................................................. 48

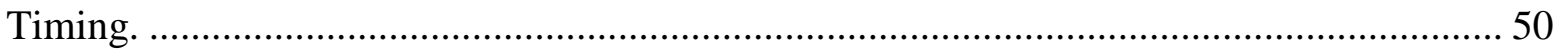

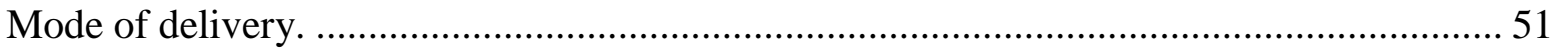

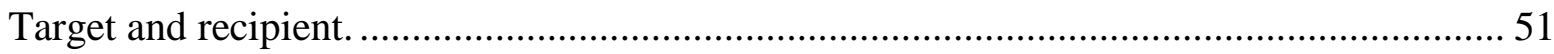

Interventionist, research assistants, and credentials ......................................................... 52

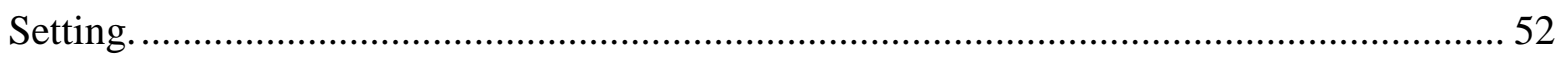

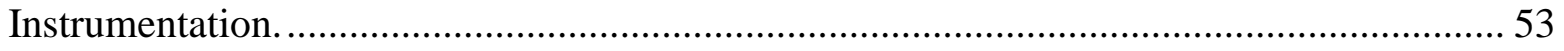

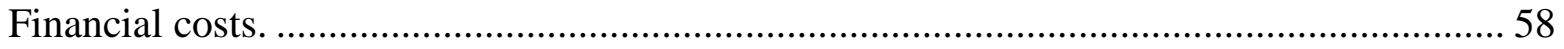

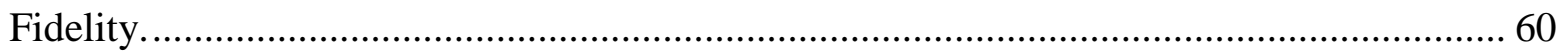

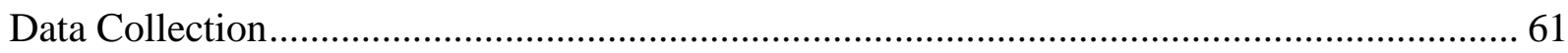

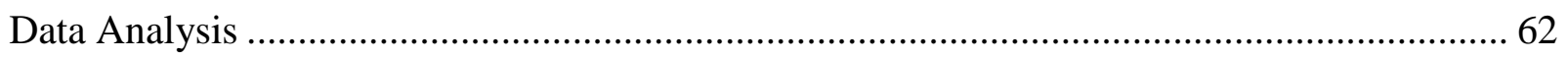

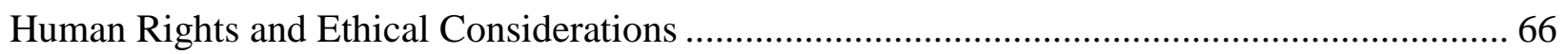

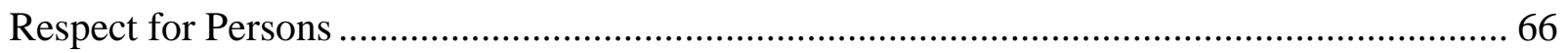

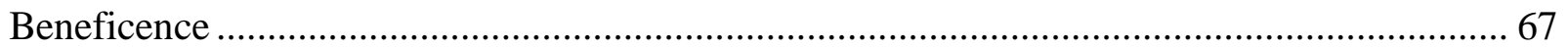

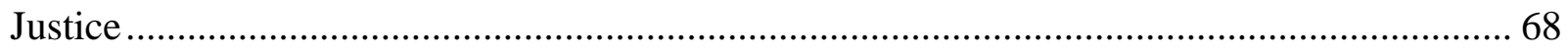

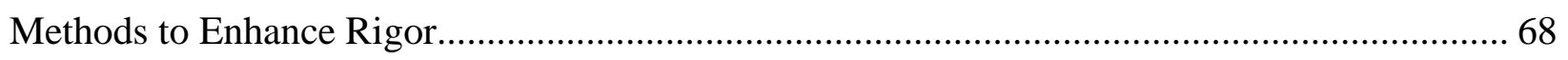

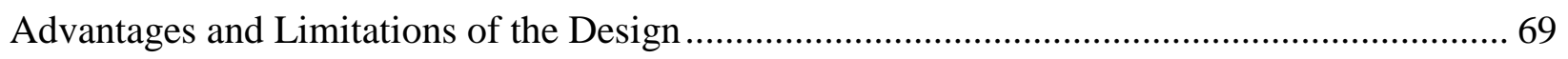

Feasibility of the Proposed CPAP-SAVER Intervention Study …………………...................... 69

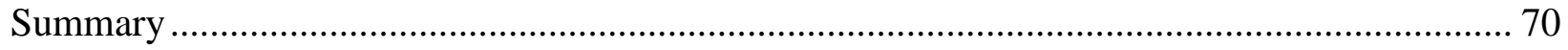

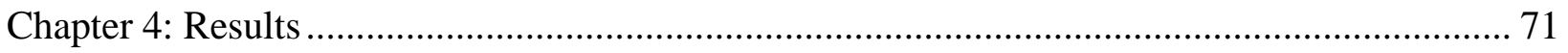

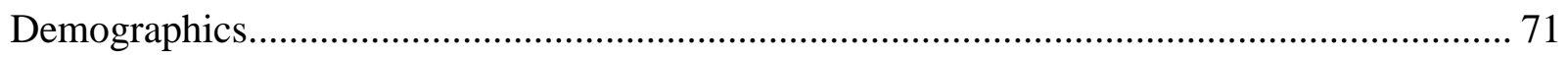

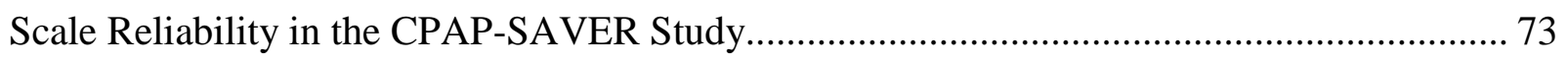

Assumptions Testing ...................................................................................................... 73

Research Question 1: Effect of the Intervention on CPAP Adherence Behavior ..................... 73

Research Question 2: Effect of the Intervention on Anxiety (as a Background Factor)........... 74

Research Question 3: Effect of the Intervention on CPAP Adherence Beliefs ......................... 75

Research Question 4: Effect of the Intervention on CPAP Adherence Attitude........................ 76 
Research Question 5: Effect of the Intervention on CPAP Adherence Subjective Norm........ 77

Research Question 6: Effect of the Intervention on CPAP Adherence PBC ......................... 77

Research Question 7: Effect of the Intervention on CPAP Adherence Intention ................... 78

Research Question 8: Prediction of CPAP Adherence Intention ....................................... 78

Research Question 9: Prediction of CPAP Adherence Behavior ........................................... 79

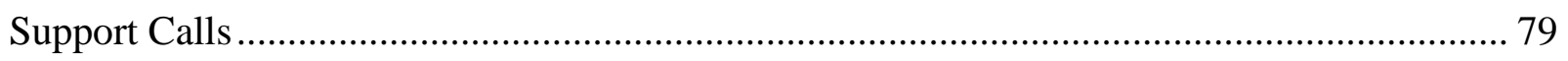

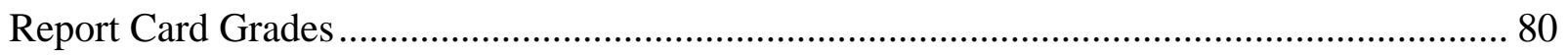

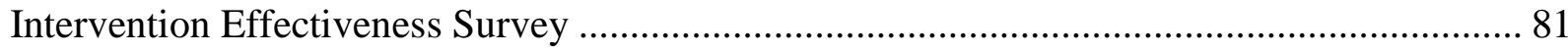

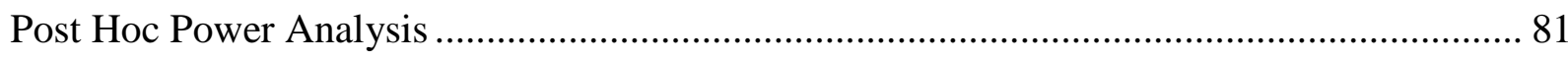

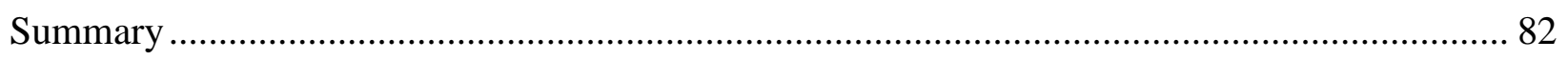

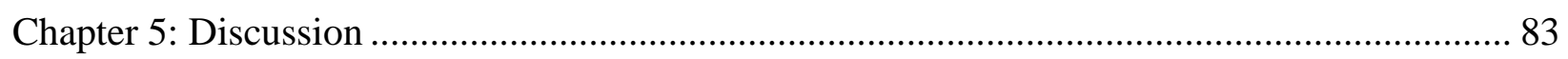

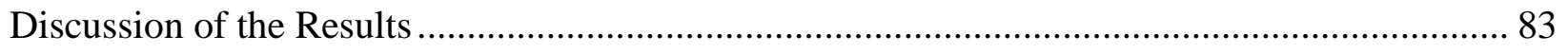

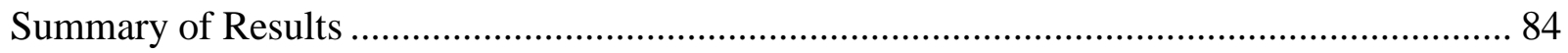

Theoretical Framework: The Theory of Planned Behavior ................................................ 85

Results in Context of the Theoretical Constructs and Previous Work..................................... 85

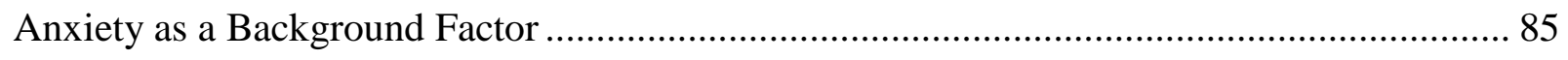

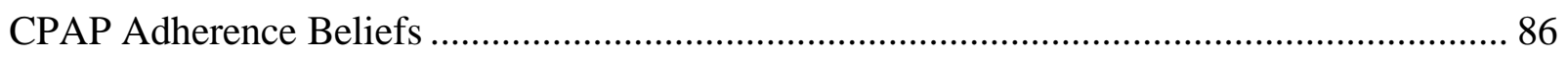

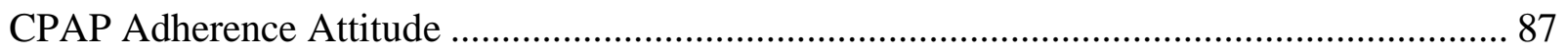

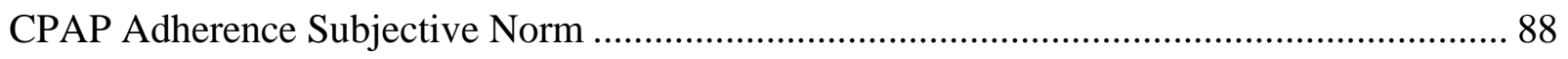

CPAP Adherence Perceived Behavioral Control ............................................................. 89

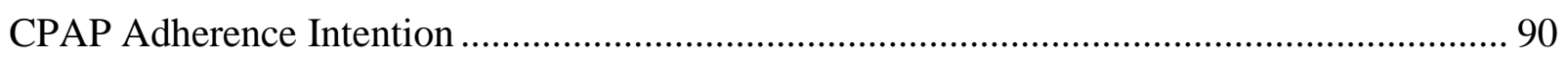

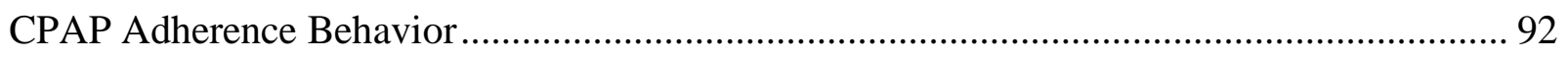

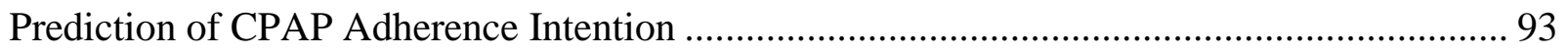

Prediction of CPAP Adherence Behavior .................................................................... 94

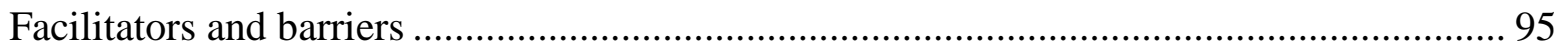

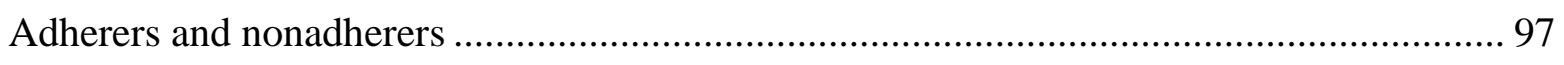

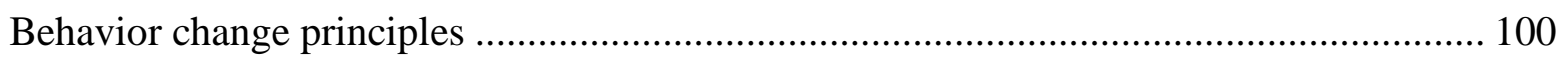

Strengths of the CPAP-SAVER Intervention Study …................................................. 101

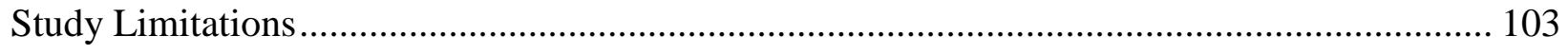

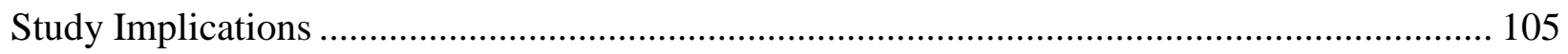

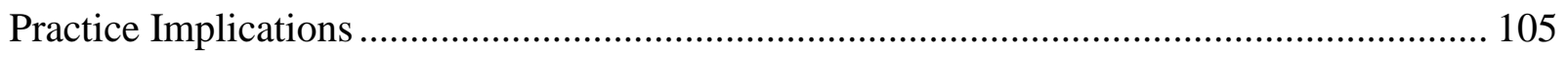

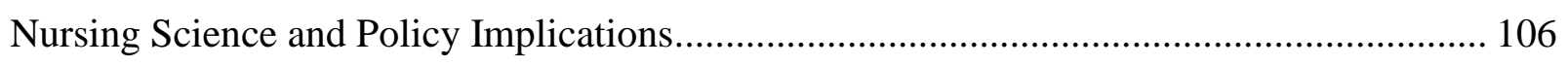


Research Implications: Recommendations for Future Research................................... 110

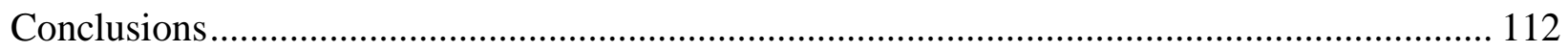

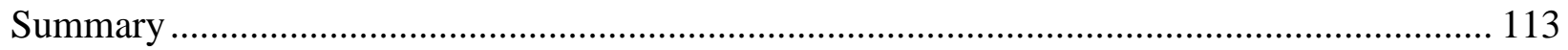

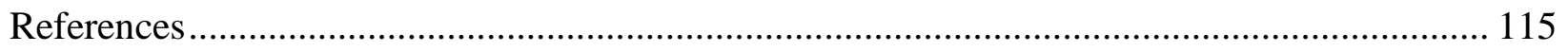

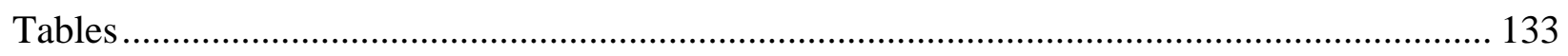

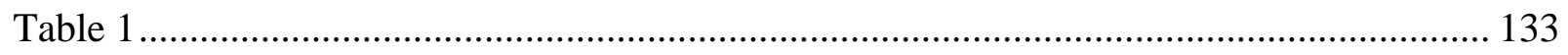

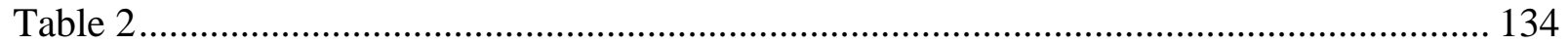

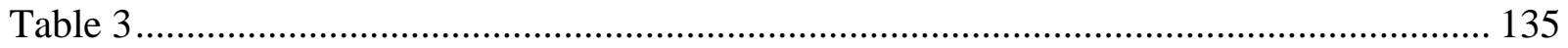

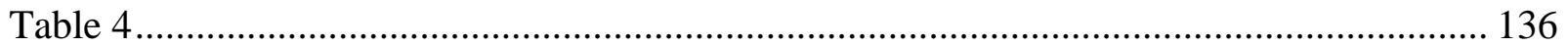

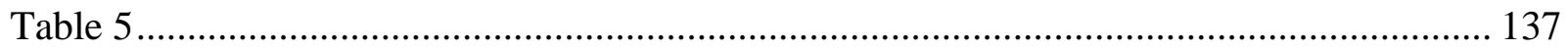

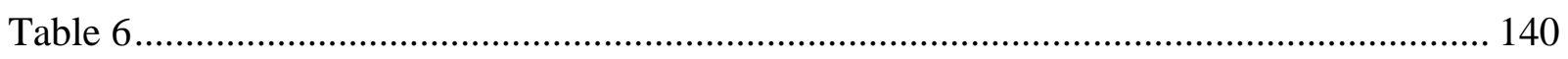

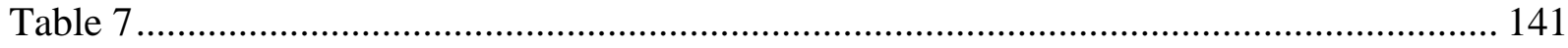

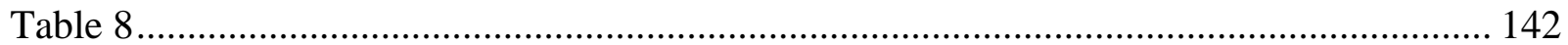

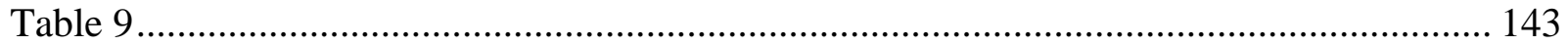

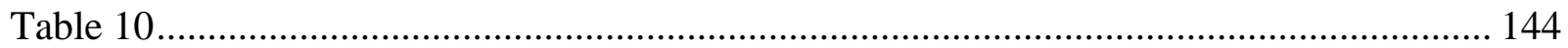

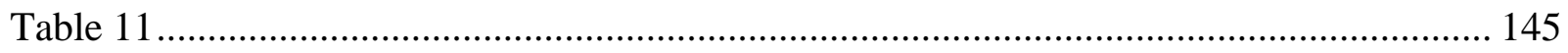

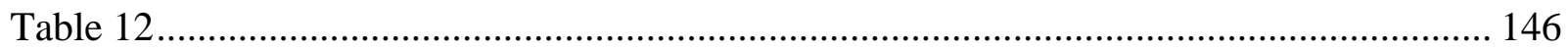

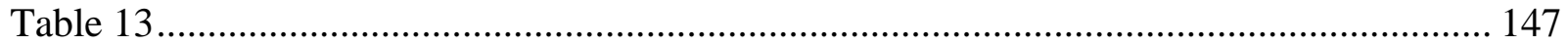

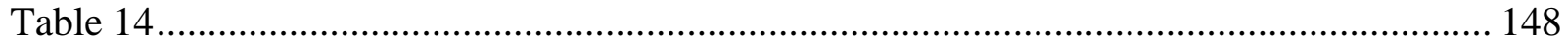

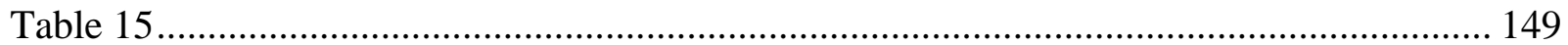

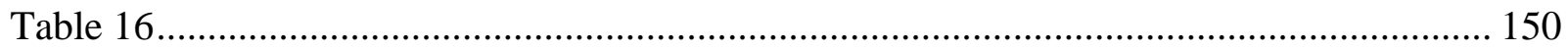

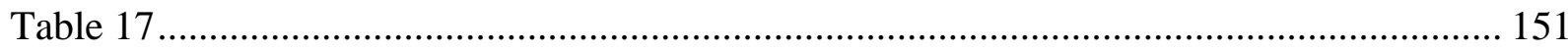

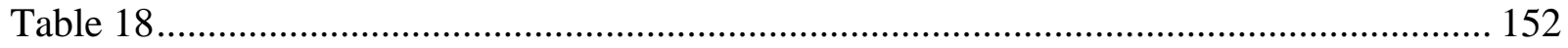

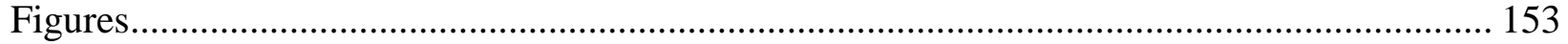

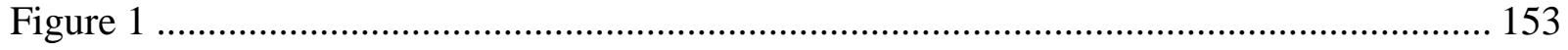

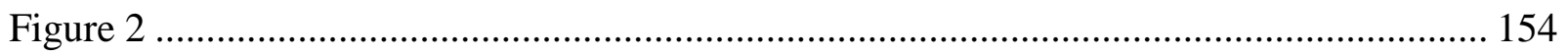

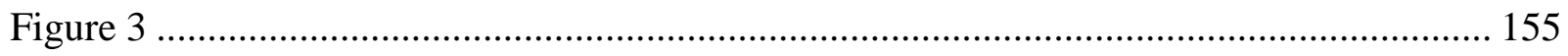

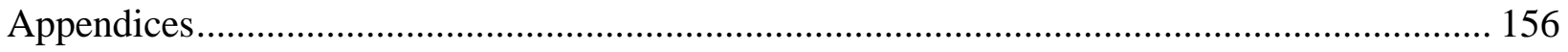

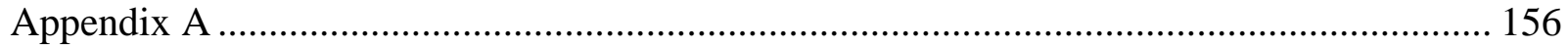

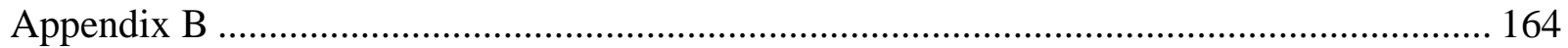

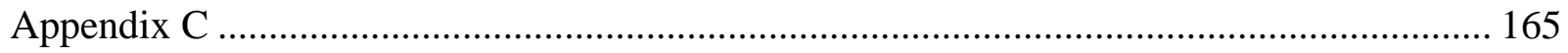

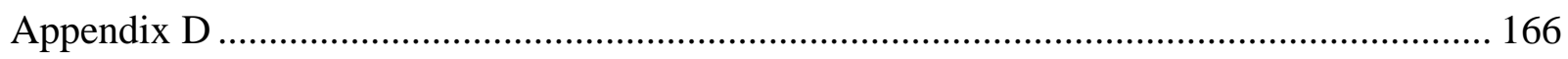




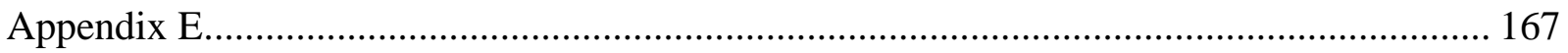

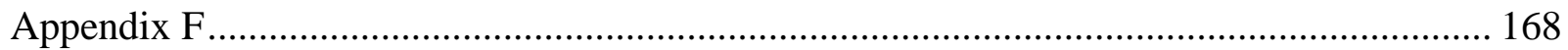

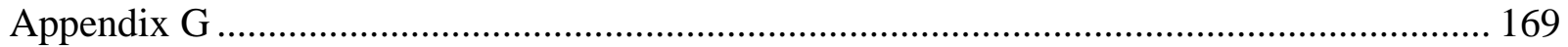

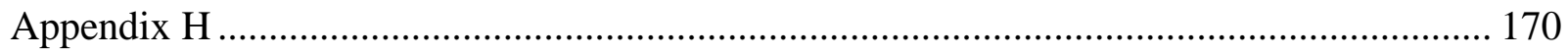

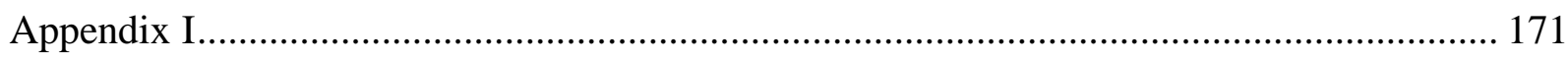




\section{Chapter 1: Introduction}

Continuous positive airway pressure (CPAP) is the gold standard, first-line treatment for obstructive sleep apnea (OSA; Epstein et al., 2009; Qaseem et al., 2013; Rakel, 2009), however, adherence is poor (Aloia, Arnedt, Riggs, Hecht, \& Borrelli, 2004; Olsen, Smith, \& Oei, 2008).

Within the first year, about $25 \%$ of all users discontinue CPAP; those who continue to use CPAP do so inconsistently and/or improperly (Aloia et al., 2004; Olsen et al., 2008). CPAP adherence rates are variable, reported anywhere from $30 \%$ to $60 \%$ (Weaver \& Sawyer, 2010). Individual (subjective) reporting of CPAP adherence is typically higher than actual (objective) measurements of use (Salepci et al., 2013). In addition, night-to-night variability is high among CPAP users, with early patterns of use predicting long-term adherence patterns (Aloia, Arnedt, Stanchina, \& Millman, 2007; Budhiraja et al., 2007; Gay, Weaver, Loube, \& Iber, 2006). The overall purpose of this study was to determine the effect of an intervention, referred to as CPAPSAVER, on one-month CPAP adherence. An intervention designed to improve early CPAP adherence may have an impact on long-term adherence and subsequent morbidity, mortality, and quality of life for adults diagnosed with OSA.

\section{Background of the Problem}

Obstructive sleep apnea (OSA) is the most common form of sleep-disordered breathing (Rakel, 2009). In the United States, approximately 6\% of all adults have moderate to severe OSA (Young, Peppard, \& Taheri, 2005), translating to at least 25 million individuals (American Academy of Sleep Medicine, 2014). Among adults 30 to 70 years of age, moderate to severe OSA is estimated to affect $13 \%$ of men and $6 \%$ of women (Peppard et al., 2013). These rates represent substantial increases in OSA prevalence over the last two decades (Peppard et al., 2013) and are expected to continue to rise alongside the increasing rates of obesity (Qaseem et 
al., 2013).

OSA is characterized by periods of complete (apnea) and partial (hypopnea) upper airway obstruction that occur repetitively during sleep, resulting in recurring oxygen desaturations and subsequent sleep arousals (Saunamaki \& Jehkonen, 2007). These events are best detected during an overnight, in-laboratory polysomnogram (PSG; Pack, 2006). During the PSG, OSA severity is determined by measuring the number of apneic and hypopneic episodes per hour, referred to as the apnea-hypopnea index (AHI). Once calculated, interpretation of the results categorizes an AHI of five to 15 as mild, over 15 to 30 as moderate, and over 30 as severe OSA (Pack, 2006). Another measure of OSA severity, the respiratory disturbance index (RDI), is a broader measure that includes respiratory effort-related arousals (RERA), events that lead to sleep arousals or microarousals but that do not fulfill the criteria for hypopneic or apneic episodes (Loube \& Andrada, 1999).

Adherence to long-term therapies is a problem with many chronic illnesses (Sabate, 2003); CPAP is no different. There are many factors that contribute to the CPAP adherence problem. CPAP, in general, is not viewed by individuals as a pleasant experience, especially since it is an intrusion into their peaceful time of sleep (Aloia, 2011). CPAP users report a stigma associated with CPAP use, specifically related to CPAP's side effects, users' beliefs that the machine is cumbersome to use, and users' beliefs that CPAP is embarrassing to wear (Ayow, Paquet, Dallaire, Purden, \& Champagne, 2009; Shapiro \& Shapiro, 2010; Willman, Igelstrom, Martin, \& Asenlof, 2012). CPAP users report many side effects, including discomfort from the pressure, mask problems, claustrophobia, machine noise, nasal congestion, mouth and nasal dryness, eye irritation, skin abrasions and ulcerations, and tooth and jaw pain (Aloia, Arnedt, et al., 2007; Salepci et al., 2013; Sawyer, Gooneratne, et al., 2011; Willman et al., 2012), many of 
which deter its use. Advances in CPAP technology have addressed some of these issues, with the use of ramping and flexible pressures, humidification, and smaller, more comfortable masks; however, more focus is needed on these problems. Since many issues relate to the machine and the mask, problem-solving strategies must be implemented early and regularly in the treatment process, not only to efficiently and effectively identify and ameliorate these issues, but to promote a more positive CPAP experience. However, this relies on the initiative of the CPAP user. Patience and persistence on the part of the user are often not present, but required (Aboussouan, Zahand, \& Podmore, 2010), further compounding the CPAP adherence problem. Poor CPAP adherence contributes to increased morbidity, increased mortality, and increased healthcare burden, with annual costs in the billions (Kapur, 2010); it is predicted that the impact of poor adherence will grow as the burden of chronic disease increases (Sabate, 2003). As a result of poor CPAP adherence, individuals with OSA may experience many physical and psychological sequelae that impact daily functioning and decrease quality of life. Daytime symptoms, such as excessive daytime sleepiness (EDS) and headaches (Epstein et al., 2009; Rakel, 2009), as well as dry and sore throat (Brostrom et al., 2007), are commonly reported by individuals with OSA. Comorbidities, including cardiovascular disease, cerebrovascular disease, and diabetes, also occur, adding to the burden caused by chronic disease (Glebocka, Kossowska, \& Bednarek, 2006; Pack, 2006; Rakel, 2009). Untreated OSA increases the risk of resistant hypertension and cardiovascular events, such as myocardial infarction, atrial fibrillation, and heart failure (Park, Ramar, \& Olson, 2011), resulting in tremendous economic burden on the healthcare system and a two-fold increase in medical costs (compared with controls) related to cardiovascular disease alone (Tarasiuk \& Reuveni, 2013). There is a twofold increase for those with mild to moderate OSA and a three-fold increase for those with severe 
OSA in all-cause mortality, compared to those with no sleep-disordered breathing (Young et al., 2008). Moderate to severe OSA is also associated with a higher risk of stroke and epilepsy (Park et al., 2011). Increased perioperative risks are prevalent among those with untreated OSA, including difficult intubation, exaggerated respiratory depression from anesthetics and analgesics, cardiac dysrhythmias, and longer hospital stays (Park et al., 2011). Psychological effects associated with untreated OSA are numerous and include anxiety, irritability, impaired concentration, depressed mood, memory loss, and neural alterations (Canessa et al., 2011; Kjelsberg, Ruud, \& Stavem, 2005; Kumar et al., 2009; Rakel, 2009). In addition, the effects of untreated OSA have impact on the individual's ability to function, with increased risks for traffic, work-related, and domestic accidents (Akashiba et al., 2002). These factors, individually and collectively, have a negative impact on the OSA sufferer's overall quality of life, potentially leading to interpersonal problems within partner, family, social, and work relationships (Reishtein et al., 2006). Treatment with CPAP has been shown to reverse the trend of increasing healthcare utilization by OSA patients and provides long-term health benefit (Tarasiuk \& Reuveni, 2013).

Another factor that has impact on OSA outcomes is obesity. Obesity is present in approximately $70 \%$ of individuals with OSA (Andrews \& Oei, 2004). OSA rates are higher in the US compared to other countries, such as Israel, Italy, and Sweden (Andrews \& Oei, 2004), possibly due to higher obesity rates. In fact, US males aged 15 and over rank fifth in world obesity rates (World Health Organization, 2010). Obesity imposes additional mechanical and central nervous system effects on the airway and breathing mechanisms (Schwartz et al., 2008), compounding the problem of worsened decreased upper airway neuromuscular activity noted with OSA (McClean, Kee, Young, \& Elborn, 2008). Increased pharyngeal fat deposits, reduced 
operating lung volumes, and increased airway collapsibility that occur with obesity add to this effect (Kapur, 2010; McClean et al., 2008; Schwartz et al., 2008). The effects of obesity on airway and breathing mechanisms, coupled with the effects of inadequately treated OSA, may promulgate a cyclic effect, possibly increasing morbidity and mortality risks, as well as healthcare and economic burden, in this population.

\section{Significance of the Study}

The CPAP-SAVER intervention study has implications for nursing science, patient outcomes, and health policy. Intervention studies aimed at improving CPAP adherence are needed (Sawyer, Gooneratne, et al., 2011; Stepnowsky et al., 2013; Weaver \& Sawyer, 2010). According to Weaver and Sawyer (2010) and Sawyer, Gooneratne, et al. (2011), CPAP adherence is a complex, multifactorial issue that demands the development of similarly designed approaches, involving education, support, anticipatory guidance, and early, frequent follow-up. Many existing interventions, while varied in strategy and effectiveness, are not theory based. In addition, the researchers implementing the studies do not describe any focus on educating participants about the OSA airway-brain mechanisms or their OSA-CPAP numbers.

The CPAP-SAVER intervention was based on the constructs of the Theory of Planned Behavior (TPB; Ajzen, 1985, 1991). The intervention was designed to: (a) promote a favorable CPAP attitude by educating the intervention participants about the OSA airway-brain mechanism and OSA risks-CPAP benefits through the use of an airway model, video, and education sheet; (b) promote a favorable subjective norm regarding CPAP adherence by providing support telephone calls; and (c) improve perceived behavioral control (perceived controllability and selfefficacy) and disease awareness by implementing an OSA-CPAP report card. Most of the CPAP-SAVER intervention was initiated within the first week of the participant's CPAP use, a 
critical time for CPAP users. The first week of CPAP therapy is a critical time for users because adherence patterns are reported to be established within this time frame (Gay et al., 2006); a stable pattern of first-week CPAP use has been shown to be predictive of longer-term CPAP adherence, as far out as six months (Aloia, Arnedt, et al., 2007; Budhiraja et al., 2007). In addition, a successful CPAP intervention should consider the typologies of adherers and nonadherers (Sawyer, Deatrick, Kuna, \& Weaver, 2010; see Table 1), as well as Abraham and Michie's (2008) taxonomy of behavior change techniques (see Table 2). The proposed CPAPSAVER intervention study was designed around these concepts - components, timing, and approach - with the intent to advance nursing science in the area of CPAP adherence intention and CPAP adherence behavior among adults with OSA.

Addressing issues related to CPAP adherence may result in improved long-term patient health outcomes. It has been noted that adherence may have a greater impact on health than improvements in specific medical treatments, thus, healthcare professionals should be trained in issues regarding adherence and deliver interventions to optimize it (Sabate, 2003). Improved CPAP adherence may have long-term implications for improved morbidity, decreased mortality, decreased healthcare burden, and decreased healthcare costs, as well as improved quality of life, for adults with OSA prescribed CPAP. Improved CPAP adherence and subsequent OSA management may impact other chronic diseases, such as those of the cardiovascular, cerebrovascular, neurological, and endocrine systems, especially since OSA has been shown to be an independent risk factor for these morbidities (Park et al., 2011). In addition, management of these comorbidities may improve with CPAP adherence and result in enhanced quality of life for adults with OSA.

This study has implications for health policy, including the establishment of a protocol 
for how patients are educated about OSA/CPAP and attention on CPAP adherence as an important issue impacting management of other chronic diseases. Over the past six years, the author conducted pilot work and multiple observations in sleep centers, physicians' offices, and home medical supply facilities. For patients diagnosed with OSA and prescribed CPAP, it was noted that education about OSA and its pathophysiology was lacking and that standard care focused on the CPAP machine and its use. Disease-focused patient education is important for management of chronic disease, including OSA, especially in the early stages of diagnosis and treatment. Focus on the OSA airway-brain mechanism, the risks associated with untreated OSA and the benefits of CPAP, and the OSA-CPAP numbers may enhance the OSA-CPAP teachinglearning process and CPAP adherence. With success of the CPAP-SAVER intervention, the groundwork for a protocol for OSA-CPAP patient education may be established, further tested, and ultimately recommended to the American Academy of Sleep Medicine for national consideration and adoption.

More nursing involvement in CPAP adherence intervention may highlight nursing's perspective of and contributions to the issue of CPAP adherence. Subsequently, this may promulgate increased nursing presence in national and global OSA-CPAP initiatives. Nursing's presence brings a unique understanding of patient partnership, connection, engagement, the health experience as it is defined and lived, and meaningful events and patterns that emerge in relationship-centered care (Newman, Smith, Pharris, \& Jones, 2008). In addition, nurses possess “... a broad appreciation of health needs, an understanding of how factors in the environment affect the health of clients and their families, and insight into how people respond to different strategies and services" (Benton, 2012, p. e2). Nursing's involvement in developing healthcare initiatives is essential to improving patients' access to quality, cost-effective care; enhancing 
patient health; and shaping health policy (Benton, 2012). However, nursing's presence in OSAand CPAP-related health policy development is lacking. In a global initiative by the World Health Organization (Sabate, 2003), experts in many areas of chronic disease examined implications of adherence to long-term therapies. The 38-member study committee included experts in the fields of many chronic illnesses, including asthma, cancer, depression, diabetes, epilepsy, and hypertension, however, did not include experts in the area of sleep medicine. In addition, nursing was underrepresented, with only two members. Another task force, the Adult Obstructive Sleep Apnea Task Force of the American Academy of Sleep Medicine (Epstein et al., 2009), was comprised of physicians and dentists, but nursing was not represented. Nursing's contribution to both research and health policy brings a different perspective to patient education and adherence, and is lacking in the area of OSA.

\section{Theoretical Framework}

The CPAP-SAVER intervention was developed based on the framework of the TPB (Ajzen, 1985, 1991; see Figure 1); the theory was also the guiding framework for testing the intervention (see Figure 2). TPB postulates that personal (attitude), social (subjective norm), and environmental or internal (perceived behavioral control) factors guide the process of behavioral intention and eventual action (Ajzen, 2011). Background factors, such as age, gender, and ethnicity, potentially influence the process of belief formation in these areas (Ajzen, 2005). An overview of the theory's assumptions, each construct of the theoretical framework, and the theory's application to the proposed CPAP-SAVER intervention follows.

\section{TPB Assumptions}

The TPB postulates five major assumptions inherent throughout the model. These assumptions were first recognized within the work on the Theory of Reasoned Action (Fishbein 
\& Ajzen, 1975), and further evolved with the TPB. The major assumptions are: (a) a human usually behaves in a sensible manner; (b) a human's behavior may not be completely under his voluntary control; (c) an individual considers the consequences and implications of his action/ behavior to decide whether or not to do something; (d) intention and behavior are highly correlated, in that whether or not the person decides to do or not do something (the intention) is strongly related to whether or not the person actually does something (the behavior; in fact, an individual's intent to perform or not perform a behavior is the most important determinant of action); and (e) an individual intends to perform a behavior when he or she: evaluates it positively, experiences social pressure to perform the behavior, and has opportunities and means to do so (motivational component; Ajzen, 2005). These assumptions underlie the TPB theoretical framework and provide the basis for the model's components.

\section{TPB Constructs}

The major constructs of the TPB are background factors, attitude, subjective norm, perceived behavioral control, intention, and behavior (Ajzen, 1991). Background factors may influence beliefs an individual holds regarding his/her attitude, subjective norm, and perception of behavioral control (see Figure 2). An individual's attitude, subjective norm, and perceived behavioral control may influence his/her behavioral intention. If a strong enough intention occurs, subsequent behavioral action may result (Ajzen, 2011; see Figure 1).

Background factors. Personal, social, and information factors may impact an individual's behavioral, normative, and control beliefs (Ajzen, 2005; see Figure 2) and should be considered in the intention-behavior process. Personal factors, such as general attitude, personality disposition/traits (including anxiety), values, emotions, and intelligence, may have an impact on beliefs and eventual intention and behavior. Ajzen (2005) proposes that general 
personality characteristics, including emotional stability (i.e. anxious versus calm, nervous versus poised), proceed to more narrowly defined behavioral tendencies and may result in specific response tendencies. Age, gender, ethnicity, religion, education level, and income are social factors which may influence beliefs. Information factors, such as knowledge, experience, and media exposure, should also be considered in the intention-behavior process, but may be more difficult to measure. Ajzen (2005) used dotted lines in the model to show the relationship between background factors and beliefs (see Figure 2); he postulated that even though background factors may influence an individual's beliefs, there are no necessary connections between them. Background factors may influence an individual's attitude, subjective norm, and perceived behavioral control (Ajzen, 2011).

Attitude. Attitude is defined as the favorable (positive) or unfavorable (negative) evaluation or appraisal of performing the behavior in question (Ajzen, 2005). Attitude toward a behavior is determined by accessible beliefs and consequences of behavior (behavioral beliefs), as well as the person's evaluation of the outcomes associated with the behavior and the strength of the associations. If an individual holds the belief that performing a behavior will lead to mostly positive outcomes, then he/she has a more positive (favorable) attitude toward the behavior and is more likely to intend to adhere. Consequently, the belief that performing a behavior will lead to mostly negative outcomes leads to a more negative (unfavorable) attitude toward the behavior and less likelihood of the individual to intend to adhere. Thus, the individual learns to favor behaviors believed to have largely desirable consequences and form unfavorable attitudes toward behaviors associated with mostly undesirable consequence (Ajzen, 1991).

Attitude is a combined sum of responses to an object, including cognitive, affective, and 
conative domains (Ajzen, 2005). Cognitive responses reflect one's perceptions of and thoughts about the attitude object. Affective responses reflect evaluations of and feelings toward, as well as physiological reactions to, the attitude object. Conative responses refer to the behavioral inclinations, intentions, commitments, and actions with respect to the attitude object. These three components, although defined independently, make up the single construct of attitude (Ajzen, 2005).

Subjective norm. According to the TPB, subjective norm is the perceived social pressure to perform or not perform a behavior (Ajzen, 2005). Subjective norm is influenced by a person's beliefs that specific individuals or groups approve or disapprove of him/her performing the behavior (normative beliefs) or that these social referents themselves engage or do not engage in the behavior. Influential individuals or groups include parents, spouse, close friends, coworkers, and, depending on the behavior, experts such as physicians. A person who believes that most referents think he/she should perform the behavior will perceive social pressure to do so. If the referents disapprove, the subjective norm puts pressure on the person to avoid performing the behavior (Ajzen, 2005).

Perceived behavioral control. The perception of behavioral control is the generalized belief that one's outcomes are self-controlled, as opposed to being controlled by external factors such as powerful others or chance (Ajzen, 2005). Behavior is strongly influenced by a person's confidence in his/her ability to perform (Ajzen, 1991). Perceived behavioral control reflects the extent to which the individual believes the performance of the behavior is within his/her control (Ajzen, 1991) and is assumed to be a function of beliefs about the presence or absence of factors that facilitate or impede behavior performance (Ajzen, 2005). Perceived behavioral control relates to both self-efficacy (confidence) and perceived controllability, and can influence 
behavior indirectly (through intentions) or directly (see Figure 1). As stated by Bandura (2004, p. 145):

Self-efficacy beliefs shape the outcomes people expect their efforts to produce. Those of high efficacy expect to realize favorable outcomes. Those of low efficacy expect their efforts to bring poor outcomes. Self-efficacy beliefs also determine how obstacles and impediments are viewed. People of low efficacy are easily convinced of the futility of effort in the face of difficulties. They quickly give up trying. Those of high efficacy view impediments as surmountable by improvement of self-management skills and perseverant effort. They stay the course in the face of difficulties.

The perception of behavioral control is influenced by beliefs based on past experience with behavior, second-hand information about the behavior, observing experiences of acquaintances and friends, and by other factors that increase or decrease the perceived difficulty of performing the behavior in question (Ajzen, 2005). It also involves perception of the ease or difficulty of enacting the behavior (Ajzen, 1991). A greater sense of perceived behavioral control tends to increase the likelihood that the individual will carry out the proposed behavior. In fact, the stronger the person's belief about his/her ability to implement the behavior, the more likely he/she is to intend to implement the behavior (Ajzen, 2005).

Another issue impacting the sense of behavioral control relates to resources and opportunities. The more required resources and opportunities a person thinks he/she possesses, and the fewer obstacles or impediments he/she anticipates, the greater should be the perceived control over the behavior (Ajzen, 2005). A person who believes that he/she has neither the resources nor the opportunities to perform a certain behavior is unlikely to form strong behavioral intentions to engage in the behavior, even if he/she holds favorable attitudes toward 
the behavior and believes that important others would approve of him/her performing the behavior. Overall, the fewer obstacles the individual perceives and the more resources and opportunities the individual believes he/she possesses, then the higher the individual's confidence level, the stronger the perception of behavioral control, and the higher his/her capacity to carry out the behavior (Ajzen, 2005).

Intention. Intention is defined as how hard the person is willing to try, how much of an effort he/she is planning to exert, in order to perform the behavior (Ajzen, 1991). Intentions are assumed to capture the motivational factors that influence a behavior. The stronger the intention to engage in a behavior, the more likely should be its performance. Behavioral intention can find expression in behavior only if the behavior is under volitional control (if the person can decide at will to perform or not perform the behavior; Ajzen, 1991). It is assumed that motivation and ability interact in their effects on behavior achievement, so intentions would be expected to influence performance to the extent that the person has behavioral control. Thus, performance should increase with behavioral control to the extent that the person is motivated to try (Ajzen, 1991). Based on the theory's premise, one's intention can be predicted with considerable accuracy by measuring attitude toward the behavior, subjective norms, and perceived behavioral control (Ajzen, 2011).

Behavior. Behavior is the manifest, observable response in a given situation with respect to a given target (Ajzen, 2006). According to the TPB, a behavior is defined by four elements: action (the behavior itself), target (source to which the action is directed), context (circumstance in which it is performed), and time (when it is expected to occur). For example: In the case of adherence to CPAP use every night while sleeping, the action is adherence, the target is CPAP use, the context is while sleeping, and the time is every night. The theory assumes that human 
behavior is reasoned or planned (Ajzen, 2011). Planned behavior is influenced by attributes of attitude, subjective norm, and perceived behavioral control, brought about by behavioral, normative, and control beliefs, respectively. The TPB proposes that an individual's attitude, subjective norm, and perception of behavioral control follow spontaneously from these beliefs, produce a relative behavioral intention, and result in actual behavior.

\section{Major Constituents of the Study}

\section{Purpose and Aims}

The overall purpose of this study was to determine the effect of the CPAP-SAVER intervention on one-month CPAP adherence in a sample of adults (aged 18 or older) with newly diagnosed OSA receiving CPAP treatment for the first time (CPAP naïve). The following aims were proposed:

1. Examine the effect of the CPAP-SAVER intervention (compared to standard care) on one-month CPAP adherence behavior;

2. Examine the effect of the CPAP-SAVER intervention on anxiety (as a background factor);

3. Examine the effect of the CPAP-SAVER intervention on CPAP adherence beliefs;

4. Examine the effect of the CPAP-SAVER intervention on CPAP adherence attitude;

5. Examine the effect of the CPAP-SAVER intervention on CPAP adherence subjective norm;

6. Examine the effect of the CPAP-SAVER intervention on CPAP adherence perceived behavioral control;

7. Examine the effect of the CPAP-SAVER intervention on CPAP adherence intention;

8. Determine if one-month CPAP adherence attitude, CPAP adherence subjective norm, 
and/or CPAP adherence perceived behavioral control are predictive of one-month CPAP adherence intention; and

9. Determine if one-month CPAP adherence intention is predictive of one-month CPAP adherence behavior.

\section{Research Questions and Hypotheses}

The research questions aligning with the purpose and aims of the CPAP-SAVER intervention study were:

1. What is the effect of the CPAP-SAVER intervention (compared to standard care) on one-month CPAP adherence behavior? It is hypothesized that the CPAP-SAVER intervention group will demonstrate significantly higher one-month CPAP adherence behavior rates than the standard care (control) group.

2. What is the effect of the CPAP-SAVER intervention on anxiety as a background factor? It is hypothesized that the CPAP-SAVER intervention group will demonstrate a significant decrease in anxiety scores over time compared to the standard care (control) group.

3. What is the effect of the CPAP-SAVER intervention on CPAP adherence beliefs? It is hypothesized that the CPAP-SAVER intervention group will demonstrate a significant increase in CPAP adherence belief scores over time compared to the standard care (control) group.

4. What is the effect of the CPAP-SAVER intervention on CPAP adherence attitude? It is hypothesized that the CPAP-SAVER intervention group will demonstrate a significant increase in CPAP adherence attitude scores over time compared to the standard care (control) group. 
5. What is the effect of the CPAP-SAVER intervention on CPAP adherence subjective norm? It is hypothesized that the CPAP-SAVER intervention group will demonstrate a significant increase in CPAP adherence subjective norm scores over time compared to the standard care (control) group.

6. What is the effect of the CPAP-SAVER intervention on CPAP adherence perceived behavioral control? It is hypothesized that the CPAP-SAVER intervention group will demonstrate a significant increase in CPAP adherence perceived behavioral control scores over time compared to the standard care (control) group.

7. What is the effect of the CPAP-SAVER intervention on CPAP adherence intention? It is hypothesized that the CPAP-SAVER intervention group will demonstrate a significant increase in CPAP adherence intention scores over time compared to the standard care (control) group.

8. Are one-month CPAP adherence attitude, CPAP adherence subjective norm, and/or CPAP adherence perceived behavioral control predictive of one-month CPAP adherence intention? It is hypothesized that one-month CPAP adherence attitude, CPAP adherence subjective norm, and CPAP adherence perceived behavioral control will be significantly predictive of one-month CPAP adherence intention.

9. Is one-month CPAP adherence intention predictive of one-month CPAP adherence behavior? It is hypothesized that one-month CPAP adherence intention will be significantly predictive of one-month CPAP adherence behavior.

\section{Definitions, Operationalization, and Measures for Variables}

The variables that were measured in this study were CPAP adherence behavior, CPAP adherence intention, CPAP adherence attitude, CPAP adherence subjective norm, CPAP 
adherence perceived behavioral control, CPAP adherence beliefs, and CPAP background factors, including anxiety. How each variable was defined, operationalized, and measured in the study is presented in Tables 3 and 4. The actual instruments used to measure the variables are presented in Appendix A.

CPAP adherence, as defined by the Centers for Medicare and Medicaid Services (2013), is the use of the positive airway pressure (PAP) device for four or more hours per night for $70 \%$ of nights during a consecutive 30-day period anytime during the first three months of initial use. Since the CPAP-SAVER study was one month in duration, CPAP adherence was operationalized as CPAP use for four or more hours per night for $70 \%$ of the nights (five out of seven nights and 21 out of 30 nights; see Table 3).

Standard care was operationalized in the study as a control group. Standard care was defined as the basic OSA and CPAP teaching and follow-up provided by the respiratory therapist/CPAP educator employed by the home medical supplier (HMS). Standard care focused on the CPAP machine set-up, use, and maintenance; resolving side effects as a result of CPAP use; machine problem solving/technical issues; and CPAP adjustments based on smart card readings at one month, as required by third-party payers, and as needed based on patient assessment.

\section{Method}

A randomized controlled trial (RCT) design was implemented to determine the effect of the CPAP-SAVER intervention (compared to standard care) on CPAP adherence intention and CPAP adherence behavior. Participants were randomly assigned to either the intervention or standard care group. Upon consent, baseline measures of each variable were collected from each participant. Participants assigned to the intervention group received the airway model, video, 
education sheet, and report card components before their standard care; participants randomly assigned to the control group received standard care only (see protocol, Table 5). Support calls by the author (as the investigator) were made to participants in the intervention group at CPAP mid-week one and CPAP mid-week two. CPAP adherence behavior and other CPAP use data for each participant, regardless of group assignment, were collected from the smart card (or wirelessly) at one week (7 nights of CPAP use) and one month (30 nights of CPAP use). Upon completion of the 30 nights, post-measures of each variable (with the exception of demographics), using the same instruments, were collected from each participant.

\section{Summary}

The overall purpose of this study was to determine the effect of the CPAP-SAVER intervention on one-month CPAP adherence in a sample of adults (aged 18 or older) with newly diagnosed OSA receiving CPAP treatment for the first time (CPAP naïve). Moderate to severe OSA affects at least 25 million adults, and contributes to increased morbidity, increased mortality, increased healthcare burden, and billions in healthcare costs. CPAP is the gold standard treatment for OSA, however, adherence is poor. Poor CPAP adherence has many physical and psychological ramifications for adults with OSA. Despite machine and mask improvements and myriad interventions, poor CPAP adherence persists. CPAP adherence is a complex, multifactorial issue that demands a similarly-designed intervention approach, considering the typologies of CPAP adherers and nonadherers as well as the taxonomy of behavior change techniques. CPAP-SAVER is a multidimensional, theory-based intervention based on critical concepts identified in the literature. Based on the TPB, the CPAP-SAVER intervention was designed to improve CPAP adherence intention and behavior by: (a) promoting a favorable attitude regarding CPAP adherence by educating participants about the OSA airway- 
brain mechanism through the use of an airway model, video, and education sheet; (b) promoting the development of a favorable CPAP subjective norm by conducting theory-based support phone calls to intervention participants; and (c) improving perceived behavioral control (perceived controllability and self-efficacy) and disease awareness through the use of an OSACPAP report card. A RCT to examine the effect of the CPAP-SAVER intervention in a sample of CPAP-naïve adults with newly diagnosed OSA was implemented, with the intent to promote improved CPAP adherence intention and behavior and advance nursing science in the field of OSA-CPAP research. 


\section{Chapter 2: Review of the Literature \\ Literature Search Process}

A systematic literature search was conducted to gather research related to CPAP adherence interventions and the use of the Theory of Planned Behavior (TPB) in CPAP adherence and other research. In addition, literature was gathered regarding the measurement of the constructs of attitude, subjective norm, and perceived behavioral control in OSA-CPAP populations. The components of CPAP adherence studies, especially the use of support; education, including the use of visual aids such as anatomical models, videos, and written materials; and objective data presented to participants in the form of a report card or progress report, were also explored.

A search of CINAHL, MEDLINE, PsycARTICLES, and PsycINFO between the years 2005 and 2016, with English language and adult-age limits, was conducted in each of the following areas. Using the keywords CPAP adherence and intervention, the literature search yielded 62 results. To collect literature related to the use of the TPB in CPAP adherence research, the keywords Theory of Planned Behavior with CPAP adherence, CPAP, and continuous positive airway pressure were entered; the search yielded no results. However, a search of the literature using the keywords Theory of Planned Behavior, intervention, and prediction, resulted in 66 studies. A search of the TPB constructs - attitude, subjective norm, perceived behavioral control, and intention - as keywords paired with CPAP yielded 23, none, one, and 15 result(s), respectively. Paired with the keyword CPAP, self-efficacy (24 studies), health support (40 studies) and peer pressure (four studies) yielded additional results. Review of each study's title and abstract were conducted to determine final inclusion based on the area of study. Ancestry and descendancy approaches were also used to identify additional relevant 
studies. In total, 24 studies of CPAP adherence interventions and their components, five studies of TPB-based health-related interventions, and 20 studies of the TPB constructs in OSA-CPAP samples were synthesized for this review.

\section{Literature Review and Synthesis}

\section{CPAP Adherence Interventions}

Twenty-four studies reporting CPAP adherence interventions conducted in adults aged 18 and over who were CPAP naïve were collected for this review. The outcome of each study focused on improving CPAP use and/or adherence among its participants. Since theory-based behavioral change interventions are thought to be more effective than those not based on theory (Stepnowsky et al., 2013), and theoretical linkage is essential in fully understanding the evidence generated by research (Conn \& Groves, 2011), the author limited her synthesis to the twelve studies reporting a theoretical framework. Of the 12 studies, four were based on Motivational Enhancement Theory (MET) alone (Aloia, Smith, et al., 2007; Lai, Fong, Lam, Weaver, \& Ip, 2014; Roecklein et al., 2010; Sparrow, Aloia, DeMolles, \& Gottlieb, 2010) and one in conjunction with the Health Belief Model (HBM; Olsen, Smith, Oei, \& Douglas, 2012). One study was based on Social Cognitive Theory (SCT; Bartlett et al., 2013) and one study was based on Cognitive Behavioral Theory (CBT; Stepnowsky, Palau, Gifford, \& Ancoli-Israel, 2007). One study was based on both SCT and CBT principles (Richards, Bartlett, Wong, Malouff, \& Grunstein, 2007). Triandis’ Interpersonal Theory was the basis for two studies (Smith, Dauz, Clements, Werkowitch, \& Whitman, 2009: Wang, He, Wang, Liu, \& Tang, 2012). The additional studies were based on Prospect Theory (Trupp, Corwin, \& Ahijevych, 2011) and the Health Action Process Approach (HAPA; Deng, Wang, Sun, \& Chen, 2013). The literature search results for the inclusive years did not yield any studies using the TPB in CPAP adherence 
research.

The studies were conducted in the United States (six), Australia (three), and China (three). Primary researchers were in the fields of nursing (four), medicine (four), psychiatry (two), and psychology (two). In the majority of the studies, a control group - usually standard care - was utilized, with the exception of a pilot study testing a sleep apnea self-management program (Stepnowsky et al., 2007). With the exception of two pilot studies with small numbers of participants (Roecklein et al., 2010 [ $N=28]$; Stepnowsky et al., 2007 [ $N=15]$ ), sample sizes for final data analyses ranged from 55 to 234 participants $(M=129.2)$ who were mainly middleaged, white men (probably due to the high prevalence of OSA among that population). Most of the studies were narrowed in focus, and particularly highlighted education, behavioral change, or both. Participants were mainly educated about OSA and CPAP basics; behavioral change principles based on the theoretical frameworks previously discussed were noted and included motivational interviewing, CBT sessions, and SCT sessions. Research questions were commonly answered by conducting chi square, t-tests, and analysis of variance (ANOVA) testing. Surprisingly, most of the studies reported CPAP use in hours instead of using CPAP adherence as the outcome (dependent variable). Results of the theory-based intervention studies varied.

MET. MET-based interventions have demonstrated mixed success. Olsen et al. (2012) compared a motivational interview nurse therapy (MINT) intervention which involved two 30minute sessions and one 20-minute booster session to a standard care group $(N=100)$. They found that CPAP use (in hours/night) in the MINT group was significantly higher than the standard care group at one month $(4.85$ vs. $3.25, p=.003)$, two months $(4.73$ vs. $3.22, p=.005)$, and three months $(4.63$ vs. 3.16, $p=.005)$, but not at 12 months $(4.21$ vs. $3.00, p=.061)$. In a 
sample of 100 participants, Lai et al. (2014) tested a MET-based intervention that included a video about OSA and CPAP, a 20-minute brief MET session, and a ten-minute call in the first week to provide follow-up and build confidence. The investigators found that the MET group had better CPAP use than the standard care group (4.4 hours/night vs. 2.4 hours/night; CI 1.3, $2.8, p<.001$, Cohen's $d=1.33$ ) and a fourfold increase in the number meeting the definition of CPAP adherence (four or more hours per night for $70 \%$ of the nights; OR $=4.3$, CI 2.0, 9.0, $p<$ .001) at three months. Based on the same theory, Sparrow et al. (2010) tested a telemedicine intervention $(N=234)$, referred to as telephone-linked communication CPAP (TLC-CPAP). The system utilized an interactive voice system (digitized human speech) to assess CPAP perception, use, and goals, and also to provide motivational feedback and counseling through weekly calls for the first month and monthly calls for 12 months. They found that TLC-CPAP participants used CPAP more hours per night than an attention control group at 6 months (median 2.4 vs. 1.48) and 12 months (median 2.98 vs. 0.99). Using the definition of adherence as four or more hours per night, by the end of 12 months the TLC-CPAP group had a 30\% higher CPAP use rate $-44.7 \%$ of the intervention group vs. $34.5 \%$ of the attention control group $(p=.006)$. However, in a study by Roecklein et al. (2010) which involved mainly low-income, African American women, there was no difference in CPAP average daily use, $F(1,19)=0.00, p=.99$, or total CPAP use hours, $F(1,20)=.15, p=.71$, between the performance feedback intervention group and the standard care control group $(N=28)$ at three months. Aloia, Smith, et al. (2007) compared standard care, education, and MET. The education and MET groups involved two 45minute sessions beginning one week after CPAP had commenced. Results showed that the three groups $(N=142)$ differed in their rates of CPAP discontinuance (use of one hour or less per night for two consecutive weeks), $\chi^{2}(N=142)=6.62, p=.04$. By the end of 13 weeks, $61 \%$ of 
the SC group, $68 \%$ of the education group, and $67 \%$ of the MET group were using CPAP an average of four or more hours per night; $32 \%$ of the SC group, $45 \%$ of the education group, and $41 \%$ of the MET group were using CPAP an average of six or more hours per night. Based on the synthesis of these studies, MET-based interventions were not consistently effective.

CBT and SCT. CBT- and SCT-based interventions have also demonstrated mixed success. Pilot work to test a sleep-apnea self-management program (SASMP) developed by Stepnowsky et al. (2007) resulted in 11 out of the 15 participants demonstrating adherence at least four hours per night measured at one month, with a mean CPAP use of $5.5 \pm 2.5$ hours per night. Richards et al. (2007) tested a CBT- and SCT-based intervention (two, one-hour CBT sessions and video/booklet education) compared to standard care $(N=96)$. The investigators reported mean nightly usage at 28 days as 2.9 hours per night longer in the intervention group compared to standard care $(t=5.4, p<.001)$; in addition, they found that $77 \%$ of the intervention group participants, compared to $31 \%$ of the standard care participants, were adherent at least four hours per night $\left(\chi^{2}=18.5, p=.002\right)$. Conversely, Bartlett et al. (2013) found no differences in CPAP use between their SCT or social interaction (SI) groups $(N=206)$ at one week, one month, three months, or six months, even after adjusting for AHI and selfefficacy. As was found in the literature review of MET-based CPAP interventions, the CBT/SCT-based interventions were highly varied in sample sizes and adherence outcomes.

Triandis' Interpersonal Theory. The studies based on Triandis' theory both resulted in improved CPAP use at one month. Wang et al. (2012) compared a standard care group, an education (ED) group (four hours of education involving use of a brochure and a video), a progressive muscle relaxation (PMR) group (12 weekly sessions of PMR and an audio compact disc), and an ED+PMR group (receiving both) in a sample of 152 participants. The investigators 
found that the ED+PMR group had higher CPAP adherence at four weeks, $\chi^{2}=10.48, p=.001$, eight weeks, $\chi^{2}=9.21, p=.002$, and 12 weeks, $\chi^{2}=8.14, p=.004$, compared to the standard care group. Smith et al. (2009) had similar effective outcomes in their music/habit-forming intervention $(N=97)$; more participants in the intervention group (89\%) compared to the control group $(55 \%)$ adhered to CPAP at one month, $\chi^{2}=14.67, p<.001$, phi coefficient $=.39$, however, there were no significant differences in adherence between the groups at three months or six months. Although one-month CPAP adherence results in both of these studies were statistically significant, there has been no additional work testing Triandis' theory in the OSA-CPAP population.

Other theories. The results of studies based on other theories, specifically Prospect Theory and HAPA, contribute to the knowledge of CPAP adherence among adults with OSA. Trupp et al. (2011) found that a Prospect-Theory based intervention which compared positivelyframed messaging (PFM) to negatively-framed messaging (NFM), had implications for OSACPAP education. They found that CPAP use was higher in the NFM group (63.14\%) than the PFM group (42.15\%), $t(52)=-2.19, p=.033$, and that $55 \%$ of the NFM group compared to $23 \%$ of the PFM group had CPAP usage $70 \%$ or greater at 30 days, $\chi^{2}=5.88, p=.015$. A HAPAbased intervention by Deng et al. (2013) demonstrated improved adherence at one month and three months for the intervention group compared to a standard care group $(N=110)$. Their stage-matched care (SMC) group received an intervention in four stages, including education about OSA and its negative consequences, aided by the use of a video and open-ended questioning; education about the proper use of CPAP, aided by the use of music, relaxation, and massage; problem solving technical issues and praising CPAP use, aided by phone calls from sleep nurses at one and three weeks and muscle relaxation; and goal setting, aided by phone calls 
from sleep nurses at six and nine weeks. Results of the study indicated that the SMC group's mean CPAP use (5.9 hours/night) was higher than the standard care group's mean (5.28 hours/night) at one month, $t=2.459, p=.016$, and at three months, $t=2.85, p=.006$ (intervention $M=5.65$, control $M=5.26$ ). Although these findings were significant, their validity and generalizability were limited in that the analyses were based on self-report CPAP numbers; sleep diaries and verbal recall were used to collect the CPAP use data since the majority of participants' machines did not have smart card technology.

Conclusion. CPAP adherence, theory-based interventions from 2005 to present tended to be unidimensional and were highly varied in their theoretical frameworks, sample sizes, focus on education, behavioral change techniques, measurement of CPAP use and/or adherence, and effectiveness. The interventions that employed theory-based sessions as a behavioral change modality, such as MET, CBT, or SCT, resulted in mixed success and often involved timely or lengthy sessions that may have contributed to participant burden. Other theory-based studies were complex to implement and/or involved nightly tasks for the participant that they may or may not have completed. Ultimately, these factors may have contributed to the diverse CPAPadherence outcomes noted in the synthesis of the studies' results. No CPAP adherence studies based on the TPB were reported in the literature. A time-efficient, low-burden, TPB-based intervention may be effective in improving one-month CPAP adherence outcomes.

\section{TPB-Based Health-Related Interventions}

Upon abstract review of the 66 TPB studies collected in the literature search, the author noted that the TPB has been used extensively in healthcare-related studies, to predict behaviors such as asthma treatment adherence (Blackwell, 2005), exercise maintenance (Ahmad et al., 2014), and intention to maintain mammography adherence (O’Neill et al., 2008). Of the 66 
studies, five studies described the testing of TPB-based, health-related interventions in adults aged 18 and over and, thus, were synthesized for this review. The studies were conducted in Australia (three; Kothe, Mullan, \& Butow, 2012; Milton \& Mullan, 2012; White et al., 2012), the United Kingdom (one; McConnon et al., 2012), and the United States (one; Montanaro \& Bryan, 2014), all by primary researchers in the field of psychology. Sample sizes ranged from 45 to 515 participants $(M=220)$; three out of the five samples were comprised mainly of young, white females attending universities. Three of the five studies compared the intervention to a control group.

Foci. The foci and other characteristics of the TPB-based studies varied. Most of the interventions promoted behavior: Fruit and vegetable intake (Kothe et al., 2012), food safety (Milton \& Mullan, 2012), regular physical activity and healthy eating (White et al., 2012), and preparatory condom use (Montanaro \& Bryan, 2014); an additional study focused on the prevention of weight regain after weight loss (McConnon et al., 2012). The researchers tended to measure the TPB constructs of attitude, subjective norm, perceived behavioral control, and intention similarly, via the direct measurement approach, however, used varied numbers of questions for each construct. Internal consistency of the attitude, subjective norm, perceived behavioral control, and intention questionnaires used in the studies ranged from Cronbach's alphas of $.72-.93, .59-.79, .44-.82$, and $.66-.91$, respectively.

Outcomes. The behavioral outcomes in the TPB-based studies were measured using ttests, ANOVA, and multivariate ANOVA statistical analyses; regression and structural equation modeling were used if the TPB model was being tested. Many of the studies used self-report in the outcome variable, such as fruit and vegetable intake the previous day (Kothe et al., 2012), the number of times participants had prepared foods hygienically over the past seven days (Milton \& 
Mullan, 2012), and condom use behaviors (Montanaro \& Bryan, 2014), which somewhat limited the findings' validity and generalizability. For most of the studies, the TPB constructs predicted a significant amount of variance in intention; the amount of variance explained by the TPB constructs, collectively, ranged from $14 \%$ of the preventing weight gain intention scores (McConnon et al., 2012) to a high of 55.1\% of the fruit and vegetable intake intention scores (Kothe et al., 2012). Subsequently, intention was a significant predictor of behavior in most of the studies; intention explained anywhere from $16.8 \%$ of the variability in fruit and vegetable intake (Kothe et al., 2012) to $27.9 \%$ of the variability in food safety behaviors (Milton \& Mullan, 2012). With the successful use of the TPB in these samples, the author hypothesizes that the theory will be applicable in the OSA-CPAP population.

Conclusion. The TPB has been extensively used in a variety of healthcare-related studies as a framework for interventions, especially interventions where behavior change is expected. The TPB constructs were measured via the direct approach, with overall acceptable internal consistency reliability. Most TPB-based studies reviewed for this synthesis resulted in significant outcomes of intention and behavior. The TPB has not been tested in the OSA-CPAP population. However, based on its success in other health-related areas, the author proposes that an OSA-CPAP intervention based on this theoretical framework may impact CPAP intention and adherence behavior.

\section{Measurement of the TPB Constructs in OSA-CPAP Samples}

Since the TPB has not been tested in an OSA-CPAP sample, the author searched the literature for the measurement of the theory's constructs of attitude, subjective norm, perceived behavioral control, and intention as they related to CPAP adherence behavior in OSA samples. The author found a total of 20 different studies (note that some of the researchers measured more 
than one of the constructs, so the following numbers will not total 20): seven measured attitude, four measured subjective norm (referred to as support or health support), 10 measured perceived behavioral control (referred to as self-efficacy), and six measured intention (referred to as motivation or willingness). A systematic, integrative, literature review described concepts related to attitude and support (Ward, Hoare, \& Gott, 2014). The studies were conducted between 2005 and the present, with samples of adults aged 18 and over who were diagnosed with OSA and prescribed CPAP; one study by Smith, Lang, Sullivan, and Warren (2004a) was included since the article described the instrument being used in the current study. The studies' methodological bases and empirical findings were synthesized for this review. No qualitative studies were found that explored background factors; thus, background factors were reviewed in the quantitative section only. Beliefs, as related to each construct, were incorporated throughout the synthesis.

\section{Methodological: Qualitative}

Attitude. Qualitative methodology has been used to explore factors influencing CPAP acceptance and adherence, including facilitators and barriers to CPAP treatment adherence (Brostrom et al., 2010), and illness and treatment attitude, beliefs, and perceptions (Tyrrell, Poulet, Pepin, \& Veale, 2006; Tzischinsky, Shahrabani, \& Peled, 2011). Differences in the perceptions of CPAP adherers and nonadherers as they relate to the OSA diagnosis and CPAP treatment were also explored, utilizing a mixed-methods, nested design and directed content and across-case analyses (Sawyer et al., 2010). In addition, Ward et al. (2014) conducted a systematic, integrative, literature review in which they explored both qualitative and quantitative data regarding CPAP experiences from users' perspectives.

Subjective norm. The literature review resulted in three qualitative studies that 
explored subjective norm-related concepts in the OSA-CPAP population. A comparative case study was conducted using semistructured interviews to explore factors influencing the use and nonuse of CPAP (Ayow et al., 2009). Dickerson and Akhu-Zaheya (2007) used a hermeneutic phenomenological approach to gather narratives for interpretive analyses to understand the lifechanges experiences by individuals diagnosed with OSA while adjusting to CPAP use. Support, both personal and professional, as a facilitator/barrier to CPAP adherence has also been explored (Brostrom et al., 2010; Ward et al., 2014).

Perceived behavioral control. No qualitative studies directly focused on perceived behavioral control, or self-efficacy, and CPAP adherence were identified in the literature. However, a directed content analysis conducted by Sawyer et al. (2010) identified perceived selfefficacy as a determinant of health behavior between adherers and nonadherers. Work by Brostrom et al. (2010) explored CPAP adherence facilitators and barriers, including self-efficacy. No other qualitative work in this area was noted.

Intention. No qualitative studies that directly pertained to CPAP intention were identified in the literature. However, some studies explored motivation as a facilitator of CPAP adherence (Brostrom et al., 2010; Dickerson \& Akhu-Zaheya, 2007). An additional study explored factors that influenced participants' motivation for purchasing or not purchasing a CPAP device (Tzischinsky et al., 2011).

\section{Methodological: Quantitative}

Background factors. Background factors, including age, weight, gender, and marital status, have been explored in the OSA-CPAP population. These factors were usually studied as predictors of CPAP adherence (Budhiraja et al., 2007; Poulet et al., 2009; Ye et al., 2012). In state of the science reviews conducted by Saunamaki and Jehkonen (2007) and more recently by the author (Shapiro, 2014), anxiety was found to be common among those with OSA. OSA 
symptoms have been shown to be predictors of anxiety in a population-based sample (Shapiro, Culp, \& Chertok, 2014). Anxiety has also been studied as a predictor of CPAP nonadherence (Kjelsberg et al., 2005). Background factors, including age, weight, gender, marital status, and anxiety, may have an impact on individuals' beliefs and subsequent decision-making regarding their CPAP adherence intention and behavior.

Attitude. The concept of attitude has been covered more extensively in the OSA-CPAP quantitative literature, especially related to CPAP adherence. Instruments to assess attitude, knowledge, and beliefs about OSA and CPAP have been developed and tested, including the Apnea Beliefs Scale (ABS; Smith et al., 2004a) and the Attitudes to CPAP Inventory (ACTI; Brostrom, Ulander, Nilsen, Svanborg, \& Arestedt, 2011). Attitude has also been explored as a predictor of CPAP adherence (Poulet et al., 2009). Attitude and its impact on CPAP expectations and use have also been examined (Ward et al., 2014).

Subjective norm. Quantitative studies examining subjective norm concepts tend to focus on significant others, especially the spouse or bed partner, and peers as opposed to healthcare providers. Intensive support by healthcare providers and its effect on patient CPAP use was explored in earlier intervention work (Hoy, Vennelle, Kingshott, Engleman, \& Douglas, 1999). Extra early support during the first week of CPAP was also tested in an intervention to examine its effect on CPAP adherence at one month, six months, and 12 months post-CPAP initiation (Lewis, Bartle, Watkins, Seale, \& Ebden, 2006).

Perceived behavioral control. Perceptions of behavioral control, or self-efficacy, have been studied extensively among individuals with OSA prescribed CPAP. Descriptive studies examining self-efficacy as a predictor of adherence have been conducted (Aloia, Arnedt, Stepnowsky, Hecht, \& Borelli, 2005; Baron et al., 2011; Sawyer, Canamucio, et al., 2011; Ye et al., 2012). Self-efficacy has also been measured in intervention studies designed to improve 
CPAP adherence (Deng et al., 2013; Lai et al., 2014; Olsen, Smith, Oei, \& Douglas, 2008; Trupp et al., 2011).

Intention. Four studies examined motivation or willingness to adhere to CPAP. Predictors of treatment adherence, such as readiness and motivation to change (Aloia et al., 2005), willingness after a short CPAP trial (Kreivi, Maasilta, \& Bachour, 2014), and intention to use (Lai et al., 2014), have been explored among new CPAP users. Level of health motivation and knowledge about OSA as a predictor to purchase a CPAP device has also been studied (Tzischinsky et al., 2011).

\section{Empirical: Qualitative}

Attitude. CPAP users' beliefs about CPAP influence their experiences with CPAP, and often times are primed by healthcare professionals to be negative, that CPAP is a difficult and problem-oriented therapy (Ward et al., 2014). Thus, preconceived attitude toward and expectations of CPAP use influence the actual CPAP experience; going into the treatment with a positive mindset has been found to improve confidence about the CPAP therapy and encourage perseverance (Ward et al., 2014). Positive attitude toward CPAP treatment was reported by participants to be a facilitator for their CPAP adherence and negative attitude toward CPAP treatment as a barrier to treatment in several studies (Brostrom et al., 2010; Sawyer et al., 2010; Ward et al., 2014), even to the point of affecting the decision to purchase a CPAP device in the first place (Tzischinsky et al., 2011). Positive CPAP attitude has been shown to be influenced by a higher OSA knowledge level, however, many individuals with OSA do not have a clear understanding of the nature of OSA, its life-threatening consequences (Tyrell et al., 2006), or the need for CPAP treatment (Brostrom et al., 2010) and, thus, may have poor CPAP adherence motivation. In addition, CPAP experience difficulties, such as machine issues and side effects, 
may impact users' adherence to treatment. Adherent CPAP users describe positive beliefs in their ability to use CPAP despite these difficulties (Sawyer et al, 2010).

Subjective norm. The presence of supportive relationships has been reported by CPAP users as an influencing factor on their adherence (Ayow et al., 2009). Conversely, nonusers have identified that the absence of supportive relationships was a contributing factor toward not using their CPAP device (Ayow et al., 2009; Brostrom et al., 2010), including poor support from healthcare providers (Brostrom et al., 2010; Dickerson \& Akhu-Zaheya, 2007). In addition to healthcare providers, loved ones and peers have been found to be influential in creating the social environment supportive of CPAP success (Ayow et al., 2009). Support in the form of receiving positive feedback from others has also been shown to help individuals persist with their CPAP treatment (Dickerson \& Akhu-Zaheya, 2007). Adherent CPAP users have reported that they rely heavily on support from both their spouses and healthcare providers; they have also reported that trust in their healthcare providers develops through support and this trust is an additional promoter of their CPAP adherence (Brostrom et al., 2010).

Perceived behavioral control. Perception of behavioral control has been shown to have a significant effect on long-term CPAP adherence. CPAP adherers have described generally positive perceived self-efficacy regarding their future CPAP use (Sawyer et al., 2010). Adherers have reported that positive belief in their ability to use CPAP at the outset of treatment helped build their confidence as they adjusted to life with CPAP and the change in their daily routine as a result (Sawyer et al., 2010). Belief in capacity to manage CPAP treatment and positive effects from it were reported facilitators to CPAP adherence; this reciprocal relationship between selfbelief/self-efficacy and attitude plays an important role in promoting a positive CPAP attitude (Brostrom et al., 2010). On the other hand, nonadherent CPAP users have described negative 
experiences early in treatment, either during the initial sleep study or early in home use, with subsequent negative effects on their perceived ability to use the CPAP device long term (Sawyer et al., 2010).

Intention. Recognizing and understanding the symptoms of OSA and noticing improvements gained by wearing the CPAP device served as motivation to continue to use the device (Dickerson \& Akhu-Zaheya, 2007). Individuals with improvement of initial severe symptoms reported better motivation to persist with their CPAP treatment than those who did not see improvement (Dickerson \& Akhu-Zaheya, 2007). In adjusting to CPAP treatment, patients described making a trade-off; negative aspects of the CPAP treatment were accepted as less important than the positive effects, such as sometimes feeling agitated at the treatment itself but waking up feeling better (Dickerson \& Akhu-Zaheya, 2007). Patients also reported that knowledge about apneas and nighttime oxygen desaturations frightened them and served to facilitate their adherence. Thus, thorough education about OSA may impact patients' overall CPAP attitude and subsequent adherence intention/motivation. Although less commonly explored, positive social support was also described as a motivator for CPAP use (Sawyer et al., 2010).

\section{Empirical: Quantitative Findings}

Background factors. Age, weight, gender, and marital status are demographic background factors that impact CPAP adherence. The prevalence of OSA increases with age (Kapur, 2010; Punjabi, 2008) and is especially common in the middle-aged (Rakel, 2009). CPAP adherence was shown to be directly proportional to age $(r=.25, p=.015)$ in a sample of 100 adult patients (Budhiraja et al., 2007). Poulet et al. (2009) found that younger patients in their sample tended to use CPAP less than older patients; they noted that patients under a median 
age of age 58 years who also had maladaptive beliefs about their OSA disease and treatment (ABS score less than 84.5) were at three times higher risk of being CPAP nonadherent ( $\mathrm{OR}=$ 3.32, CI 1.22, 9.01, $p=.02$ ). Excess body weight is common among those with OSA (Punjabi, 2008) and is a strong causal factor for sleep-disordered breathing (Peppard et al., 2013).

However, in studies by Budhiraja et al. (2007), Poulet et al. (2009), and Ye et al. (2012), BMI was not associated with nor a predictor of CPAP use. Men have a greater vulnerability toward developing OSA (Punjabi, 2008) and have shown to be less CPAP adherent than women $(p=$ .06; Poulet et al., 2009). Poulet et al. (2009) found that males with worse attitudes toward OSA and CPAP (ABS score less than 84.5) had 2.37 times more risk of poor CPAP adherence $(\mathrm{OR}=$ 2.37, CI 1.01, 5.6, $p=.048)$. Marital status may also have an effect on CPAP adherence. During the first week of treatment, married patients demonstrated increased nightly use of CPAP (in hours) compared to those who were not married ( $4.2 \pm 2.8$ vs. $2.8 \pm 2.4, p=.01$; Ye et al., 2012). Even just living with someone has demonstrated an effect on adherence; CPAP users living with someone had a machine-on time of 4.5 hours compared with those living alone who had a machine-on time of 3.2 hours ( $p=.04$; Lewis, Seale, Bartle, Watkins, \& Ebden, 2004).

Anxiety is another background factor shown to influence CPAP adherence, quality of life, and physiological status among adults with OSA. Anxiety is common among those with OSA, more so in middle-aged men, and tends to improve with CPAP treatment (Shapiro, 2014). OSA symptoms, especially nonrefreshing sleep $(\mathrm{OR}=3.582$, CI $1.981,6.476, p<.001)$ and awakenings at night due to apneic episodes ( $\mathrm{OR}=2.047$, CI 1.423, 2.945, $p=.001)$, were predictive of anxiety among middle-aged men in a population-based sample $(N=1217$; Shapiro et al., 2014). High anxiety levels have been associated with nonadherence to CPAP ( $p=.005$; Kjelsberg et al., 2005). In a study of 56 patients newly diagnosed with OSA, there was a 
significant decrease in anxiety symptoms with CPAP treatment that was sustained at six months $(p=.0215)$. Anxiety may also contribute to permanent brain changes among individuals with OSA. Among the OSA-anxious population, researchers have noted permanent neurostructural brain abnormalities on magnetic resonance imaging especially in areas of the cortices, thalamus, hippocampus, and amygdala (Antic et al., 2011; Kumar et al., 2009). Anxiety has many implications for adults with OSA.

Attitude. Research supports the effect of attitude on outcomes and adherence in many chronic diseases, such as diabetes and asthma, and medication regimen (Sabate, 2003). For those prescribed CPAP, attitude has been demonstrated to be a positive predictor of adherence (Poulet et al., 2009). In a study by Poulet et al. (2009), participants with maladaptive beliefs were 2.21 times more likely to be nonadherent to CPAP (CI 1.03, 4.72, $p=.04$ ), especially males; males with a poorer attitude to health score had a 2.37 times higher risk of poor adherence (CI 1.01, 5.6, $p=.048)$. CPAP adherent individuals tend to have a more positive attitude and favorable adaptive beliefs compared to CPAP nonadherent individuals (Poulet et al., 2009). Beliefs about CPAP were antecedents to individuals' attitude toward the actual CPAP treatment (Brostrom et al., 2011). The manipulation of variables, including health beliefs, has been shown to improve health outcomes in a variety of conditions similar to OSA, such as diabetes and hypertension (Smith et al., 2004a). Subsequently, instruments to measure beliefs and attitudes toward CPAP treatment, including the ABS (Smith et al., 2004a) and the ACTI (Brostrom et al., 2011), have been developed, tested, and successfully implemented in OSA-CPAP research, to quantify these psychological constructs.

Subjective norm. There are a limited number of quantitative studies in the area of health support for CPAP users. What is known is that healthcare provider, spouse, and family support 
provided early in CPAP treatment impacts adherence rates (Hoy et al., 1999; Lewis et al., 2006). In an intervention study of 72 patients starting CPAP therapy who were randomly assigned to receive standard care or an extra early support intervention (20-minute video, week-one phone call, and extra sleep physician follow-up appointment), reattendance follow-up rates were significantly higher for the intervention group at one month $(p=.04)$, but not at six months $(p=$ $.07)$ or 12 months $(p=.17)$. However, the mean nightly CPAP use, measured at 12 months, ranged from 4.6 to 5.1 hours, meeting the definition of adherence (Lewis et al., 2006).

Perceived behavioral control. Self-efficacy has been shown to influence CPAP use, demonstrating both predictive (Aloia et al., 2005; Sawyer, Canamucio, et al., 2011) and moderating (Baron et al., 2011) effects. Lai et al. (2014) reported positive relationships between CPAP adherence and treatment self-efficacy at one month $(r=.438, p<.001)$ and three months $(r=.42, p<.001 ; N=100)$. Similar findings were reported by Baron et al. in 2011; they noted that higher self-efficacy was associated with a stronger relationship between adherence and positive affect $(\gamma=.08, \mathrm{SE}=.03, p<.01 ; N=31)$. Self-efficacy has also been shown to effect long-term CPAP adherence. In a study by Ye et al. (2012), investigators found that posttreatment self-efficacy was associated with duration of use $(r=.27, p=.035)$. A multimodal, stage-matched intervention improved self-efficacy over a three-month period (effect of time, $F=$ $155.68, p<.001, \eta^{2}=.63$; effect of group, $F=21.65, p=.001, \eta^{2}=.19$; and effect of time*group, $F=9.26, \mathrm{p}=.003, \eta^{2}=.09$; Deng et al., 2013). Kreivi et al. (2014) found that patients who continued CPAP treatment after one year had significantly higher scores on a selfefficacy questionnaire obtained prior to a CPAP trial $(p<.001)$.

CPAP education has been shown to influence self-efficacy. Self-efficacy was found to be the most influential predictor of one-week CPAP use $\left(\beta_{\text {est }}=1.52, \mathrm{SE}= \pm .53, p=.006\right)$ and 
explained almost $9 \%$ of the variance in one-week CPAP use; this influential trend continued after a disease- and treatment-specific education session $\left(\beta_{\mathrm{est}}=1.40, \mathrm{SE}= \pm .52, p=.009\right)$ and at one month $\left(\beta_{\mathrm{es} t}=1.20, \mathrm{SE}= \pm .50, p=.02\right.$; Sawyer, Canamucio, et al., 2011). In a study by Trupp et al. (2011), those individuals assigned to an intervention group to receive NFM (compared to PFM) had higher self-efficacy scores, $t(25)=2.41, p=.023$, and improved adherence (Pearson's $\left.\chi^{2}[1, N=55]=5.88, p=.015\right)$.

Intention. Providing education on the long-term consequences may increase intention/ motivation to accept and use CPAP treatment, in both the short and long term. Higher level of knowledge was a significant factor that positively affected participants' decisions to purchase a CPAP device in a study by Tzischinsky et al. in $2011(\beta=-2.98, \mathrm{SE}=1.79, p=.09)$. In addition, those with higher levels of health motivation were more apt to purchase a CPAP device than those with low motivation $(p=.02)$. Lai et al. (2014) tested a brief MET-based education program and found a significant difference in intention to use CPAP at three months (difference $31 \%$, CI $20 \%, 42 \%, p<.001)$. In a regression model to predict six-month CPAP adherence (Aloia et al., 2005), one-week measures of readiness, self-efficacy, and decisional balance were significant $(\beta=1.92, \mathrm{SE}=.47, p<.001 ; \beta=.19, \mathrm{SE}=.29, p=.043$; and $\beta=-.08, \mathrm{SE}=-.32, p=$ .036 , respectively) and accounted for $23.2 \%$ of the variance $\left(r^{2}=.23\right.$, adjusted $r^{2}=.20, F[3,69]$ $=6.66, p=.001)$. Long-term adherence has also been examined; low willingness score measured immediately after a CPAP trial predicted whether the patient would stop CPAP therapy by one year (specificity 94\%) and predicted poor adherence (use less than four hours/night; specificity 97\%; $N=580$; Kreivi et al., 2014).

Conclusion. There are many OSA-CPAP studies that have examined the TPB constructs, including attitude, subjective norm (support), perceived behavioral control (self- 
efficacy), and intention (motivation/willingness). Findings indicated that a positive CPAP attitude was a facilitator of CPAP motivation and subsequent adherence, and was especially influenced by knowledge of OSA risks. Support, especially spousal and healthcare provider, was an influencing factor on adherence, shown to be built on positive feedback and trust. Perceived behavioral control, especially self-efficacy, had a significant effect on short- and long-term CPAP adherence. Early positive experiences facilitated a more positive attitude and sense of confidence in adjusting to CPAP use. Intention to use CPAP was facilitated by education about OSA consequences, especially to highlight the positive effects of CPAP. No study has explored these constructs collectively and their impact on adherence. Thus, a single intervention study designed to impact each of the TPB constructs - attitude, subjective norm, perceived behavioral control, and intention - may result in significant improvements in CPAP adherence among adults with OSA.

\section{The CPAP-SAVER Intervention and Its Components}

The CPAP-SAVER intervention components were developed based on pilot work previously conducted by the author, the TPB constructs, and literature support. In a small sample of middle-aged and older men with OSA $(N=4)$, the author found that participants were lacking support, had a poor understanding of OSA and its associated risks, were not aware of many of the benefits of CPAP, and did not know their OSA severity or CPAP machine numbers (Shapiro \& McCrone, 2016). These findings provided impetus for the support, education, and report card components of the CPAP-SAVER intervention. The TPB provided the theoretical framework for the intervention components. According to Ajzen (2011), a behavioral intervention must target change in the behavioral, normative, and control beliefs that contribute to the formation of attitude, subjective norm, and perceived behavioral control. The CPAP- 
SAVER intervention components are designed to facilitate the development of favorable behavioral, normative, and control beliefs to impact the CPAP user's attitude, subjective norm, and perceived behavioral control. The author hypothesizes statistically significantly higher CPAP adherence intention and CPAP adherence behavior rates as a result of these components and their theoretical base:

- Airway model, video, and education sheet: Attitude

- Support calls: Subjective norm

- Report card: Perceived behavior control

Each of the 24 CPAP adherence studies conducted between 2005 and the present were analyzed for the specific components of the CPAP-SAVER intervention: Support calls (five studies), airway model (no studies), video (nine studies), education sheet (eight studies) and report card (one study). The results were synthesized for this review.

Support calls. Support calls have been used in intervention studies to promote CPAP adherence. In five studies reviewed for this synthesis (Deng et al., 2013; Lai et al., 2014; Lewis et al., 2006; Parthasarathy et al., 2013; Trupp et al., 2011), most of the calls were provided by nurses. The calls focused on identifying CPAP problems and offering advice (Lewis et al., 2006); providing positively- or negatively-framed messages about CPAP benefits and OSA risks (Trupp et al., 2011); offering clinical support, praise, and encouragement (Deng et al., 2013); and highlighting positive changes and reminding participants of OSA's negative consequences (Lai et al., 2014); in one study, peer buddies made a total of eight calls that focused on coping strategies and efficacy improvement (Parthasarathy et al., 2013). Two of the studies did not indicate the length of the call (Deng et al., 2013; Parthasarathy et al., 2013), however, other researchers reported calls that were a median of 10 minutes. The nurse calls ranged in number 
from one call placed at mid-week one of CPAP use (Lai et al., 2014; Lewis et al., 2006), to a total of four calls either made once weekly over the first four weeks (Trupp et al., 2011) or at one, three, six, and nine weeks post-CPAP initiation (Deng et al., 2013). For the interventions that involved support calls, the results tended to be significant in improving CPAP use, followup, and adherence rates. In a Cochrane review of 30 CPAP usage interventions (Wozniak, Lasserson, \& Smith, 2014), the reviewers reported that supportive interventions resulted in an increase of participants using CPAP, from 59/100 pre-intervention to 75/100 post-intervention; CPAP use per night (increase of 0.82 hours); and CPAP adherence ( $\mathrm{OR}=2.06, \mathrm{CI} 1.22,3.47$ ).

Airway model. Anatomical models have been used successfully in education, offering cognitive and psychomotor advantages over photographic images and digital media (Pawlina \& Drake, 2013). In the 24 CPAP adherence intervention studies reviewed, no researchers described the use of an airway model in the education of participants with OSA prescribed CPAP. Demonstrating the basic mechanisms of the sympathetic response that occurs with each apneic episode and the effect of CPAP in splinting the airway on an anatomical model may offer advantages in this study. The use of an anatomical model in teaching about the airway may impact the understanding of the basic pathophysiology of OSA, may promote a more favorable attitude toward CPAP therapy, and may improve CPAP adherence behavior.

Video. Video education was used extensively throughout the studies analyzed for this review. In the nine studies reviewed, the videos tended to focus on general OSA and CPAP education (Basoglu, Midilli, Midilli, \& Bilgen, 2012; Deng et al., 2013; Lai et al., 2014; Lewis et al., 2006; Trupp et al., 2011; Wiese et al., 2005); two videos included personal experiences of CPAP users learning to manage CPAP (Bartlett et al., 2013; Richards et al., 2007); and another video focused on the CPAP device and its use (Wang et al., 2012). The videos averaged 14.5 
minutes, with a range of 50 seconds to 25 minutes in duration. Results of seven of the nine studies indicated higher machine use, follow-up, and adherence rates in the intervention group compared to standard care.

Education sheet. Education sheets are commonly used in teaching patients about OSA and CPAP. In the intervention studies synthesized for this review, eight studies described the use of written materials, and included booklets (Lai et al., 2014; Richards et al., 2007), pamphlets and reminder placards (Deng et al., 2013; Smith et al., 2009), a magnet (Trupp et al., 2011), a brochure (Wang et al., 2012), and slide presentation handouts (Bartlett et al., 2013); one study referred to the educational materials as "written support" (Meurice et al., 2007, p. 38). All of the materials tended to focus on general OSA and CPAP education, but also included information about sleep and general health (Bartlett et al., 2013; Richards et al., 2007) and nightly CPAP use reminders (Deng et al., 2013; Smith et al., 2009). The majority of the interventions resulted in significantly higher CPAP use and adherence rates compared to controls. In a study by Smith, Lang, Sullivan, and Warren (2004b) using the ABS to measure apnea beliefs, individuals who participated in an educational intervention demonstrated a significant, positive improvement in apnea-related beliefs over time $(F[2,28]=4.06, p<.05)$; the participants demonstrated a tendency towards more positive beliefs and attitudes immediately post-education and their scores either improved or were sustained at three months. In addition, Wozniak et al. (2014) reported moderate quality evidence showing that short-term educational interventions resulted in modest increases in participants using CPAP, from 57/100 preintervention to 71/100 post-intervention; CPAP use per night (increase of 0.6 hours); and CPAP adherence compared to standard care (OR 1.80, CI 1.1, 2.95).

Report card. Among individuals with OSA, seeing objective data related to their 
condition has resulted in improved CPAP use (Falcone, Damiani, Quaranta, Capozzolo, \& Resta, 2014). The successful use of patient report cards has been reported in diabetes ("Physician group," 2003), dialysis (Karalis, 2001), and stroke (UCLA, 2000) management, but not in OSACPAP maintenance. In a study by Roecklein et al. (2010), personalized feedback (compared to standard care) did not improve CPAP average daily use $(F[1,19)=0.00, p=.99)$ or total hours of use $(F(1,20)=.15, p=.71)$; their feedback included AHI, RDI, average and nadir oxygen saturation, self-reported daytime sleepiness, and the risks of a car accident, high blood pressure, and high body weight, but did not include continued AHI and CPAP progress. Having knowledge of both the current $\mathrm{AHI}$ and CPAP pressure settings may remind the patient of progress he/she is or is not making, and may be encouraging, motivating, or both. A CPAP report card that documents both OSA and CPAP progress may have a significant impact on participants' perception of behavioral control of their OSA and subsequent CPAP adherence.

Conclusion. The need for support, OSA and CPAP education, and understanding of OSA-CPAP numbers were the impetus for the CPAP-SAVER intervention. Support calls, videos, and written educational materials have been used extensively and successfully in intervention studies designed to promote CPAP adherence, however, there are gaps in the literature related to the use of an airway model to educate patients about the OSA airway-brain mechanism and the use of a report card to document OSA-CPAP progress. Phone calls by a nurse conducted early after CPAP initiation have focused on problem solving, education, and clinical support, but have not been utilized to establish a subjective norm. Videos used in the studies analyzed for this synthesis focused on general OSA and CPAP education to promote CPAP adherence with general success. However, video education focused on the OSA airwaybrain mechanism was not described in the literature. Written materials, such as education sheets, 
were commonly used to educate participants. Topics typically included general OSA and CPAP basics with some focus on OSA consequences and CPAP benefits. The CPAP-SAVER intervention is comprised of these commonly used components - support calls, video, and education sheets - and focuses on the use of an airway model and a report card. Sawyer et al.'s (2010) typologies of adherent and nonadherent CPAP users (See Table 1) and Abraham and Michie's (2008) taxonomy of behavior change techniques (see Table 2) were also considered in the development of the intervention. CPAP adherence is a complex issue, and as such, a theorybased intervention utilizing a mixed-strategy approach may have a significant impact on CPAP adherence.

\section{Summary}

This chapter provided an overview of the literature review search process and a synthesis of the theoretical, methodological, and empirical underpinnings of the CPAP-SAVER study. Extensive research related to theory-based CPAP adherence interventions, TPB-based healthrelated interventions, measurement of the TPB constructs in OSA-CPAP samples, and the CPAP-SAVER intervention components was explicated and synthesized. With the prevalence of poor CPAP adherence and noted gaps in the literature, support for testing this theory-based intervention is evident. 


\section{Chapter 3: Method \\ Research Design}

The CPAP-SAVER intervention study used a RCT design. This type of design was suitable for testing the effect of an intervention (Polit \& Beck, 2012), such as the CPAP-SAVER, and examining relationships between variables, including the TPB constructs, CPAP adherence intention, and CPAP adherence behavior. This was a randomized control design with random assignment of subjects to either the intervention or standard care group. In this design, there was no random selection of a sample; a convenience sample was enrolled in the study.

\section{Population and Sample Selection}

To test the CPAP-SAVER intervention, a convenience sample of 66 adults (aged 18 and over) newly diagnosed with OSA and prescribed CPAP for the first time (CPAP naïve) was recruited from two home medical supply facilities over 10 months (January through October 2016). Flyers, posters, and word of mouth were strategies used for participant recruitment. The flyers and posters included information about the study's purpose, inclusion/exclusion criteria, and voluntary nature.

Using G*Power 3.1 (Faul, Erdfelder, Lang, \& Buchner, 2007), an a priori power analysis determined a sample size of 33 in each group (intervention and control; $N=66$ ) to achieve adequate statistical power (.80), with a .05 alpha level and medium effect size (see Figure 3). Reported adherence rates vary from $30 \%$ to $60 \%$, so the control group adherence proportion was set at .30 and the intervention group proportion was set at .60. The following criteria guided the recruitment and delimitation of the accessible population of participants:

Inclusion criteria

- Age 18 or older

- Able to read, understand, write, and speak English 
- Newly diagnosed with obstructive sleep apnea by overnight, in-lab polysomnogram

- Commencing OSA treatment for the first time

- Prescribed CPAP for OSA treatment

- Using a CPAP machine with smart card technology

- Using one of the home medical supply facilities participating in the study

- Provided informed consent

Exclusion criteria

- Requires bi-level ventilation

- Has significant craniofacial abnormalities

- Diagnosed with Down syndrome

- Diagnosed with a cognitive delay

- Diagnosed with hypotonia

- Diagnosed with a neuromuscular degenerative disorder

- Taking medication for anxiety

- Pregnant

Once a participant volunteered to enter the study, if he/she experienced mask and/or CPAP machine problems as CPAP treatment commenced, the home medical supplier worked with the participant to resolve the issues. The investigator was prepared to permit this window of time to allow resolution of the issues before data collection continued with such participants. The investigator noted that allowing this window of time would have extended the individual's participation in the study to slightly longer than a month.

\section{The CPAP-SAVER Intervention}

The CPAP-SAVER intervention was developed based on the Theory of Planned Behavior (TPB; Ajzen, 1985, 1991; see Figures 1 and 2). The author also consulted Sawyer et al.'s (2010) typologies of adherent and nonadherent CPAP users (See Table 1) and Abraham and Michie's (2008) taxonomy of behavior change techniques used in interventions (see Table 2), both to enhance the effectiveness of the intervention. In addition, recommendations by Conn (2012), Conn \& Groves (2011), and Whittemore (2009) were followed to improve the reporting and generalizability of the findings. Details about the intervention (see protocol, Table 5), 
including randomization, components, dosing, frequency, timing, delivery mode, target, recipients, interventionist and her credentials, research assistants and their credentials, setting, instrumentation, financial costs, and fidelity, are reported in the following sections.

Randomization. After consent, participants were randomly assigned to either the intervention or control group, and were masked as to group assignment. A summary of the randomization process follows:

1. The investigator prepared 33 manila clasp envelopes; the contents included a sheet with the word Intervention printed on it, two copies of the consent form, and one copy of the instruments (see Appendix A) - the demographic survey, TPB questionnaires (Francis et al., 2004), ABS (Smith et al., 2004a), and Beck Anxiety Inventory (BAI; Beck \& Steer., 1993). The envelopes were numbered sequentially from one through 33; this number served as the participant's identification number. The contents in each envelope were numbered to match the envelope.

2. The investigator prepared another 33 manila envelopes; the content included a sheet with the words Standard care printed on it, two copies of the consent form, and one copy of the instruments (see Appendix A) - the demographic survey, TPB questionnaires (Francis et al., 2004), ABS (Smith et al., 2004a), and BAI (Beck \& Steer, 1993). The envelopes were numbered sequentially from 34 through 66; this number served as the participant's identification number. The contents in each envelope were numbered to match the envelope.

3. The investigator mixed all the envelopes into one batch and shuffled them 10 times.

4. The investigator divided the shuffled envelopes into four piles, one pile for each home medical supply facility site (Sites A, B, C, and D). The initial number of 
envelopes provided to each facility was based on the average number of patients with OSA prescribed CPAP seen in one month's time:

Site A: 10

Sites B, C, and D: 8

5. The investigator delivered the specified number of envelopes to the home medical supply facilities when she conducted the initial training sessions, and trained all research assistants (respiratory therapists $[\mathrm{RT}]$ ) in the envelope's use.

The initial number of sites was four (Sites A, B, C, and D). Two sites were lost to attrition (Sites $\mathrm{C}$ and D) due to the unavailability of a respiratory therapist to provide CPAP teaching. As these two sites withdrew from the study, recruitment slowed at Site B, and recruitment progressed at Site A, unused envelopes from Sites B, C, and D were taken to Site A. Additional envelopes were provided to sites as needed; all 66 envelopes were used.

Intervention components, dosing, and frequency. The experimental group received the CPAP-SAVER intervention in addition to standard care; the control group received standard care only. The five components of the CPAP-SAVER intervention were Support calls, Airway model, Video, Education sheet, and Report card. In this study, the components were implemented by RT in the home medical supply facilities and a nurse (the author as investigator). In preparation for implementation of the study, each part of the intervention was initially rehearsed three times by the investigator to determine the estimated total time for implementation of the intervention.

Support calls. The investigator made two telephone calls to each intervention group participant: the first at CPAP mid-week one and the second at CPAP mid-week two. Each of the two calls followed the subjective-norm-based script (see Appendix B) and were approximately 
five minutes in length. The calls were mainly designed to provide support and to exert social pressure (subjective norm) to promote CPAP adherence, however, data collected during the calls (from questions five and six) was also analyzed for this study.

Airway model. The RT providing the CPAP teaching conducted an education session using an airway model (Airway Simulator Board, purchased from Anatomy Warehouse), showing the airway-brain mechanism of OSA and how CPAP acts as a splint to the airway. The education was provided following a script (see the CPAP-SAVER Intervention Study Protocol Training Manual) and was conducted during the first CPAP teaching session before the standard care. The session took approximately two minutes to complete. The intent was to promote favorable CPAP attitude formation.

Video. The RT showed the participant a three-minute, OSA-CPAP web-based video, highlighting the airway-brain mechanism, basic OSA pathophysiology, and CPAP as an airway splint (How CPAP Works, Ken Warner Remote). The video was shown during the first CPAP teaching session, following the airway model teaching session but before the standard care. The intent of the video was to supplement the airway model education in the promotion of favorable CPAP attitude formation.

Education sheet. The RT reviewed an education sheet with the participant. The education sheet, entitled The Risks of Obstructive Sleep Apnea \& the Benefits of CPAP, was designed by the investigator and highlighted the airway-brain mechanism of OSA, the risks associated with untreated OSA, and the benefits of CPAP (see Appendix C). The education sheet contained two graphics, one showing the partial and complete blockages of airflow with snoring and apnea and the other showing a comfortable-appearing, sleeping male individual wearing CPAP and his bed partner. The education sheet was written with a mix of negatively- 
and positively-framed statements, designed to promote understanding of OSA, discourage CPAP nonadherence, and promote CPAP adherence. The sheet was reviewed with the participant one time, at the start of the CPAP teaching session; the sheet was given to the participant to take home. Following a script (see the CPAP-SAVER Intervention Study Protocol Training Manual), the education sheet took about three minutes to review with the participant and was designed to promote favorable CPAP attitude formation.

Report card. The RT initiated and the nurse investigator maintained a CPAP Report Card (see Appendix D) to document the participant's OSA severity (AHI), CPAP machine settings, CPAP machine use (mask-on time collected from the machine smart card), the participant's self-evaluation of his/her CPAP progress (self-rating of grade A [demonstrates adherence], B [showing steady progress], $\mathrm{C}$ [progressing, but with much support], or $\mathrm{N}$ [not adherent]), and the RT's evaluation of the participant's CPAP progress (same grading scale). This report card was initiated after the diagnostic sleep study and updated at CPAP week one and CPAP month one (with data collected from the smart card/wireless modem). The report card took about two minutes to initiate and one minute to update each time (week one and month one), including the time it took the RT/investigator to review the smart card data and write in the numbers on the report card. The report card was developed by the investigator and was designed to promote the participant's sense of controllability and self-efficacy related to his/her CPAP use and adherence, with a visual record of his/her progress. A script (see the CPAP-SAVER Intervention Study Protocol Training Manual) was provided to guide the RT in reviewing the report card with each participant.

Timing. The five components of the CPAP-SAVER intervention were implemented at prescribed times (see Table 5). For participants in the intervention group, the components, in 
addition to standard care, were provided in the following order, with the anticipated time allotment:

Airway model, video, and education sheet: Two minutes, three minutes, and three minutes, respectively

Report card initiation: Two minutes

Standard care (initial CPAP teaching): 30 minutes on average

Support call \#1: Five minutes

Report card update \#1: One minute

Standard care (CPAP one-week follow-up smart card reading): Five minutes on average

Support call \#2: Five minutes

Standard care (CPAP one-month follow-up in person): Five minutes on average

Report card update \#2: One minute

The total amount of time it took to implement all components of the one-month CPAP-

SAVER intervention was approximately 22 minutes per intervention participant. Of the total 22

minutes, 12 of the minutes were added to the RT time with the participant (airway

model/video/education sheet time and report card initiation and maintenance) and 10 of the minutes were nurse (investigator) time with the participant (two telephone calls focused on support and review of the report card). The order of the components was based on educational principles, the intervention protocol, and standard care provision.

Mode of delivery. The CPAP-SAVER intervention involved two modes of delivery: Face-to-face and telephone. The airway/video/education sheet components were provided during one face-to-face session. The support/subjective norm component was provided via two telephone calls. The report card was first presented to the participant during his/her initial faceto-face CPAP session, reviewed with the participant via telephone during the second support call, and reviewed with the participant face-to-face at the one-month CPAP follow-up session.

Target and recipient. The target for the intervention was the accessible population of individual adults with OSA prescribed CPAP for the first time. The recipients of the intervention were those individual adults with OSA prescribed CPAP for the first time who were recruited 
and became part of the sample in the study; the intervention was delivered directly to those individuals. Each participant was compensated with a $\$ 25.00$ gift card upon completion of the baseline part of the study and $\$ 50$ in gift cards upon completion of the one-month part of the study, for a total of $\$ 75.00$. A thank you card was also provided to each recipient at both times.

Interventionist, research assistants, and credentials. The investigator was the interventionist and was responsible for most of the components of the intervention, including designing and overseeing the entire intervention, training three research assistants (RT), checking fidelity of the study, implementing the support calls, and assisting with the maintenance of the report card. The investigator who conducted this study is a master'sprepared registered nurse and educator, working on her Doctor of Philosophy $(\mathrm{PhD})$ degree in nursing research and implementing her first intervention. Her previous experience includes nine years of professional nursing in areas of medical-surgical nursing and 17 years in undergraduate nursing education. She has worked with many patients with obstructive sleep apnea and has taught students about the disease and its treatment.

Three respiratory therapists assisted the investigator in the study and were responsible for consenting participants, implementing the airway/video/education part of the intervention, initiating and updating the report card, and maintaining intervention fidelity. The research assistants were either certified or registered respiratory therapists and had extensive respiratory care experience.

Site A: Two research assistants; each with 25 years of experience (as RT and with CPAP)

Site B: One research assistant; 21 years of experience as RT and four years with CPAP Setting. The intervention was implemented within home medical supply facilities where participants voluntarily went to receive their CPAP device and training. Two facilities 
located in West Virginia were utilized. Each site had been in operation for ten or more years. Contact persons were established with each facility. Written approval was obtained from each facility.

Instrumentation. After consent, data was collected from each participants using a demographic survey; BAI (Beck \& Steer, 1993); ABS (Smith et al., 2004a); TPB questionnaires (Francis et al., 2004) tailored to measure the constructs of CPAP adherence attitude, CPAP adherence subjective norm, CPAP adherence perceived behavioral control, and CPAP adherence intention; the sleep study report, and CPAP smart card/wireless modem readings for CPAP adherence behavior. Participants were asked to complete the instruments at the home medical supply facility site before they received their initial OSA-CPAP teaching (intervention or standard care); they were also asked to complete instruments at the completion of the study when they visited the home medical supply facility site for their one-month follow-up. Throughout the study, the CPAP smart card/wireless modem data was accessed by a special card reader used by the RT in the home medical supply facilities; the investigator tracked the OSA-CPAP numbers with a $\log ($ see Appendix E).

Demographic survey. A survey designed by the investigator was used to collect background factors (see Appendix A), including age (years), weight (pounds), height (inches), gender (male or female), marital status (no or yes), bed partner status (no or yes), predominant ethnicity (White or other), highest educational level (high school or less or beyond high school), socioeconomic status (based on total annual household income), type of medical insurance provider, employment status (no or yes), shift work (no or yes), and night shift work (no or yes). Body mass index (BMI) was calculated using the weight and height data. A question about OSA-related comorbidities was also included. 
BAI. Anxiety was measured as a background factor in the CPAP-SAVER study. The BAI (Beck \& Steer, 1993) was used as a screening tool for anxiety (see Appendix A; the official instrument was purchased for use in the study). The instrument was comprised of 21 items (symptoms). Participants rated themselves on how they felt over the past two weeks on items, such as unable to relax and fear of the worst happening, using a Likert scale from zero (not at all) to three (severely - I could barely stand it). The total BAI score ranged from zero to 63 and was interpreted as: zero to seven - minimal anxiety; eight to 15 - mild anxiety; 16 to 25 moderate anxiety, and 26 to 63 - severe anxiety. In initial testing of the instrument $(N=1086)$, the inventory demonstrated high internal consistency (coefficient alpha of .92) and one-week test-retest reliability (0.75); in addition, the inventory demonstrated discriminant validity by differentiating anxious and nonanxious groups $(F[2,157]=18.60, p<.001$; Beck, Epstein, Brown, \& Steer, 1988). In a sample of OSA patients $(N=303)$, Cronbach's alpha levels of .92, .91 , and .92 for the total sample, men, and women, respectively, were reported (Sanford, Bush, Stone, Lichstein, \& Aguillard, 2008). In the same sleep sample, factor analysis with varimax rotation demonstrated a one-factor solution for the 21 items (Sanford et al., 2008). The BAI was written at the fifth-grade reading level, and takes about 10 minutes to complete and about 10 minutes to score manually; it is available in paper or web format. The cost for the scoring manual and 25 forms was $\$ 128$, and $\$ 56.40$ for each additional 25 forms (purchased through Pearson's Assessments).

ABS. The ABS was developed by Smith et al. (2004a). The scale is comprised of 24 items designed to assess OSA- and CPAP-related beliefs related to perceived impact of OSA, trust in medical staff, outcome expectations, CPAP acceptance, openness to new experiences, commitment to change, willingness to ask for help, attitude toward health, and self-confidence. 
Participants completing the instrument rated themselves on items, such as Sleep apnea has no effect on my life and If things become too much I generally don't go through with them, using a Likert scale from one (strongly disagree) to five (strongly agree); ten of the items were negatively worded to reduce response bias and, thus, were reverse coded upon scoring. The total ABS score ranged from 24 to 120, with higher scores indicating more positive attitude and beliefs towards CPAP adherence (Smith et al., 2004a). In initial testing of the instrument in a sleep apnea sample $(N=81)$, the scale demonstrated moderate internal consistency (coefficient alpha of .75) and validity in clinical and non-clinical samples $(t[41.79]=6.43, p<.01$; Smith et al., 2004a). In a subsequent intervention study testing psychological variables as predictors of CPAP adherence in a sample of adults with OSA $(N=120)$, discriminant validity was evident; participants with maladaptive beliefs (total ABS score less than 84.5) were 2.21 times more likely to be nonadherent to $\mathrm{CPAP}(\mathrm{CI}=1.03,4.72 ; p=.04)$, differentiating adherers from nonadherers (Poulet et al., 2009). The ABS was written at the sixth-grade reading level (Smith et al., 2004a), and takes about 10 minutes to complete and about 10 minutes to score manually; it is available in paper format. The scale was copied for use by the author, with no cost to use the instrument (per e-mail communication with Dr. Simon Smith dated March 15, 2015).

\section{TPB questionnaires for attitude, subjective norm, perceived behavioral control, and}

intention. The TPB-based questionnaires to measure attitude, subjective norm, perceived behavioral control, and intention were tailored to measure these constructs in this OSA-CPAP sample (see Table 4 and Appendix A) from recommendations provided by Francis et al. (2004). No permission was needed and no costs were involved to use the questionnaires. The instrument was written at the seventh-grade reading level and takes about 10 minutes to complete; it is in paper format and takes about 10 minutes to score manually. Direct-measure questions for 
attitude asked participants to rate themselves on a seven-point, bipolar adjectives scale with a single stem. To collect the total score, the negatively-worded adjectives were reverse coded and the mean of the four item scores calculated to determine overall attitude score. The total score ranged from 1 to 7 , with a higher mean indicating a more positive attitude toward the target behavior. In a study of a TPB-based intervention designed to promote healthy eating and physical activity in older adults with type II diabetes or cardiovascular disease $(N=183)$, the attitude direct-measure questions yielded Cronbach's alphas of .82-.89 for healthy eating and $.81-.82$ for physical activity (White et al., 2012). In a study comparing the success of two interventions designed to increase condom use behavior among college students $(N=258)$, one based on the HBM and the other based on the TPB (compared to an information-only control), the Cronbach's alpha for the TPB direct-measure attitude questions was .78 (Montanaro \& Bryan, 2014).

The four direct-measure, subjective norm questions asked participants about the expectations and social pressure from others to perform the behavior in question. To collect the total score, the negatively-worded adjectives were reverse coded and the mean of the four item scores calculated to determine overall subjective norm score. The total score ranged from 1 to 7 , with higher overall mean indicating more social pressure to perform the target behavior. Internal consistency (Cronbach's alpha) for the TPB-based subjective norm scale was reported between .67 in a study to predict post-anterior cruciate ligament rehab intention among 87 athletes (Niven, Nevill, Sayers, \& Cullen, 2012) to .85 in a study to predict the maintenance of physical activity among 94 people enrolled in a gym for the first time (Armitage, 2005).

Perceived behavioral control was measured with a total of four direct questions, two designed to assess self-efficacy and two designed to assess controllability (Francis et al., 2004). 
To collect the total score, the negatively-worded adjectives were reverse coded and the mean of the four item scores calculated to determine the overall perceived behavioral control score. The total score ranged from 1 to 7 , with a higher overall mean indicating a higher perception of behavioral control. In an intervention study designed to promote fruit and vegetable intake among undergraduate college students in a first-year psychology course $(N=194)$, Kothe et al. (2012) reported a Cronbach's alpha of .72 for the perceived behavioral control questionnaire. Similar results (Cronbach's alpha $=.71)$ were reported in a pilot intervention study designed to increase chlamydia testing among college students living in deprived areas $(N=253$; Booth, Norman, Goyder, Harris, \& Campbell, 2014).

Generalized intention was measured with a total of three direct questions (Francis et al., 2004). The items were designed to gather information about the participant's expectations, desires, and intent to perform the behavior in question. Unlike the other construct measures, the three intention items were positively worded so reverse coding was not necessary. The mean of the three item scores was calculated to determine overall intention score. The total score ranged from 1 to 7, with a higher mean indicating a higher intention to perform the target behavior. Internal consistency (Cronbach's alpha) for the TPB-based generalized intention scale was reported between .63 in a study to predict post-anterior cruciate ligament rehab intention among 87 athletes (Niven et al., 2012) to .72 in a study to predict treatment adherence among 117 South Africans living with diabetes and hypertension (Kagee \& Merwe, 2006).

OSA severity, CPAP setup, and CPAP use, and CPAP adherence. Initial OSA severity data (AHI and oxygen saturation nadir) was collected from the sleep study report; subsequent OSA severity and CPAP use/adherence data (including AHI and mask-on time) was collected from the CPAP machine's smart card/wireless modem. The data was obtained by using the 
smart card reader/wireless modem access within each home medical supply facility. AHI and mask-on time were the two data points that were collected from the smart card/wireless modem to be recorded on the intervention report card. AHI was measured as the average number of events per hour, and was categorized as five to 15 as mild, over 15 to 30 as moderate, and over 30 as severe (American Academy of Sleep Medicine, 2008). Mask-on time indicated adherence or not; adherence was defined as mask-on time at the prescribed pressure for at least 4 hours per night for $70 \%$ of the nights (for week one: $7 \times 70 \%=5$ nights; for month one: $30 \times 70 \%=21$ nights). CPAP machine humidification and pressure settings, as well as the type of mask the participant was wearing (full, nasal, or nasal pillows), were also noted. The investigator maintained a log of OSA- and CPAP-related data (see Appendix E).

Financial costs. The CPAP-SAVER study was completely funded by the WVU School of Nursing. The financial costs associated with the specific intervention components were minimal. The following cost itemization of the intervention component was calculated based on an intervention group size of 33 participants and accounted for a few extra copies of items:

$\begin{array}{lllll}\text { Support calls (and log) } & \begin{array}{l}\text { No cost (free long distance) } \\ \text { Black/white copying, single }\end{array} & \$ 0.00 \times 40 & \$ 0.00 \\ \text { Airway model } & \begin{array}{l}\text { Purchase of the models, } \\ \text { including 6\% tax, free } \\ \text { shipping }\end{array} & \$ 76.85 \times 4.60 & \$ 307.40 \\ & \text { No cost (free use on web) } & \$ 0.00 & \$ 0.00 \\ \text { Video } & \text { Color copying, single } & \$ 0.37 \times 40 & \$ 14.80 \\ \text { Education sheet } & \text { Black/white copying, single } & \$ 0.09 \times 40 & \$ 3.60 \\ \text { Report card } & & & \$ 329.40\end{array}$

Additional financial cost calculations included copying the recruitment materials, protocol manual, consent forms, demographic survey, TPB questionnaires (Francis et al., 2004), ABS instrument (Smith et al., 2004a), OSA-CPAP data log, and fidelity checklist, as well as 
purchasing the BAI (Beck \& Steer, 1993) manual and forms. For a sample size of 66 and accounting for a few extra copies of items, these costs (and quantities) were:

\begin{tabular}{|c|c|c|c|}
\hline \multicolumn{4}{|l|}{ Recruitment materials } \\
\hline Flyers (100) & Color copying, single & $\$ 0.37 \times 100$ & $\$ 37.00$ \\
\hline Posters, large (8) & Color, 24 x 36 & $\$ 36.99$ x 8 & $\$ 295.92$ \\
\hline \multirow[t]{4}{*}{ Protocol manual (5) } & Black/white copying, duplex, & $\$ 0.09 \times 27$ & $\$ 12.15$ \\
\hline & 27 pages & x 5 & \\
\hline & Divider tabs & $\$ 4.00 \times 5$ & $\$ 20.00$ \\
\hline & Notebooks & $\$ 4.00 \times 5$ & $\$ 20.00$ \\
\hline Consent form (140) & $\begin{array}{l}\text { Color copying, duplex, } \\
6 \text { pages, stapled }\end{array}$ & $\begin{array}{l}\$ 0.33 \times 6 \\
\times 140\end{array}$ & $\$ 277.20$ \\
\hline \multirow[t]{2}{*}{ Demographic survey (75) } & Black/white copying, duplex, & $\$ 0.09 \times 2$ & \multirow[t]{2}{*}{$\$ 13.50$} \\
\hline & two pages & x 75 & \\
\hline TPB-based questionnaires & Black/white copying, duplex, & $\$ 0.09 \times 4$ & $\$ 50.40$ \\
\hline & four $\mathrm{p}$ & & \\
\hline ABS (140) & Black/white copying, single & $\$ 0.09 \times 140$ & $\$ 12.60$ \\
\hline \multirow[t]{2}{*}{ BAI (150) } & Manual +25 record forms, & $\$ 128.00$ & \multirow[t]{2}{*}{$\$ 410.00$} \\
\hline & 125 additional record forms & $\$ 282.00$ & \\
\hline OSA-CPAP data log (75) & Black/white copying, single & $\$ 0.09 \times 75$ & 6.75 \\
\hline Fidelity checklist (60) & Black/white copying, single & $\$ 0.09 \times 60$ & 5.40 \\
\hline $\begin{array}{l}\text { Intervention/Standard care } \\
\text { sheets for envelopes ( } 75)\end{array}$ & Black/white copying, single & $\$ 0.09 \times 75$ & 6.75 \\
\hline $\begin{array}{l}\text { Envelopes for instruments } \\
\text { (140) }\end{array}$ & Manilla clasp, 100/box & $\$ 12.00 \times 2$ & $\$ 24.00$ \\
\hline \multirow[t]{3}{*}{ Thank you cards (132) } & 100/box & $\$ 20.00$ & 20.00 \\
\hline & 50/box with shipping & $\$ 15.92$ & $\$ 15.92$ \\
\hline & Labels for printing message & $\$ 8.88$ & $\$ \quad 8.88$ \\
\hline
\end{tabular}

The black/white and color copies, as well as the recruitment posters, calculated above were subsequently made by the WVU School of Nursing Office of Nursing Research; this saved the investigator $\$ 739.67$ of the anticipated costs. 
Most of the costs associated with the CPAP-SAVER intervention study were related to participants' compensation and research assistants' payments. Each of the 65 participants who completed the study were compensated with a total of $\$ 75.00$ in gift cards (total cost $=\$ 4875.00$ ) throughout the study; one participant lost to attrition only received the initial $\$ 25.00$ in gift cards (the participant's remaining \$50 in gift cards were returned to the WVU School of Nursing on January 12, 2017). Upon completion of the study, each of the three research assistants were compensated $\$ 200.00$ (total cost $=\$ 600.00)$. The final, total cost of implementing the CPAPSAVER intervention study was:

\begin{tabular}{ll} 
Intervention & $\$ 307.40$ \\
Supplies & $\$ 518.80$ \\
Participants' compensation & $\$ 4900.00$ \\
Research assistants' compensation & $\$ 600.00$ \\
\hline Total & $\$ 6326.20$
\end{tabular}

The author notes that the costs for the recruitment materials, protocol manual, consent forms, demographic survey, TPB questionnaires (Francis et al., 2004), ABS instrument (Smith et al., 2004a), BAI (Beck \& Steer, 1993), OSA-CPAP data log, fidelity checklist, and participants'/research assistants' compensations would not be incurred in the everyday implementation of the intervention in the healthcare setting. To implement the CPAP-SAVER intervention in practice, each facility would need to purchase the airway model $(\$ 76.85)$; the other CPAP-SAVER components would cost $\$ 0.55$ per individual patient (support call log, education sheet, and report card). The time investment (additional 22 minutes) would also need to be considered in the CPAP-SAVER implementation.

Fidelity. Training of the research assistants (RT) was conducted by the nurse investigator in an initial and a booster session using the CPAP-SAVER Intervention Protocol Training Manual (see separate file). Fidelity checks were completed monthly following the 
CPAP-SAVER Intervention Fidelity Checklist (see Appendix F). During each fidelity check, the investigator observed the RT while conducting an intervention session. The initial fidelity check in a site was conducted after two participants had been enrolled in that site. In the event that no participants were enrolled in a site during any particular month, no fidelity check was conducted for that month. For Site A, seven fidelity visits and five check-in visits were conducted throughout the study; for Site B, no fidelity visits were made since the site only recruited two participants, however, seven check-in visits were made.

In addition, the investigator evaluated each participant's perception of the effectiveness of the components of the intervention using an Intervention Effectiveness Survey (see Appendix G). The survey was administered to those in the intervention group upon completion of the study (one month). Data from the effectiveness survey was analyzed and interpreted in context of the overall intervention results.

\section{Data Collection}

Data for each participant was collected over one month. Pre-study quantitative data was collected from all participants and included the demographics survey, BAI (Beck \& Steer, 1993), ABS (Smith et al., 2004a), TPB-based questionnaires (Francis et al., 2004), sleep study results (AHI and oxygen saturation nadir), and CPAP machine/mask settings and details. Data was collected from the intervention group participants during the two support/subjective norm telephone calls (mid-week one and mid-week two); although the main purpose of the calls was to promote a subjective norm, data from questions three (machine and mask problems), five (most troublesome symptom of sleep apnea), and six (OSA- and CPAP-related beliefs and support) were analyzed quantitatively for the study. Quantitative data was collected from all participants at one week (via smart card/wireless modem access of the CPAP machine) and one month 
(during the return visit to the home medical supply facility); the data from the smart card/ wireless modem included the AHI and mask-on time as the indicator of adherence, as well as other CPAP use numbers. Upon completion of the study (one month), quantitative data was collected from all participants and included the BAI (Beck \& Steer, 1993), ABS (Smith et al., 2004a), TPB-based questionnaires (Francis et al., 2004), and CPAP machine/mask settings and details.

\section{Data Analysis}

As data was collected, it was entered twice into the Double Data Entry Spreadsheet (DeCoster \& Iselin, 2006) and then checked for errors upon data collection completion; the investigator compared and referred to the source data to resolve errors. Data was imported into and analyzed using Statistical Package for the Social Sciences (SPSS), version 24 (IBM, 2016). Alpha was set at .05 for all analyses. The SPSS Survival Manual (Pallant, 2016) was used as an instructional guide for the analyses. Assumptions testing was performed, as appropriate, for each statistical test (homogeneity of intercorrelations, homogeneity of variance, multicollinearity, singularity, outliers, normality, linearity, homoscedasticity, and independence of residuals). Univariate analyses (frequencies and descriptives) were conducted on all variables at baseline for the entire sample. Univariate analyses (frequencies and descriptives) were conducted on all variables at baseline for participants by group (intervention and control) to determine homogeneity of the groups. The research questions were answered with the following analyses:

1. What is the effect of the CPAP-SAVER intervention (compared to standard care) on one-month CPAP adherence behavior? A Pearson chi-square test of independence with intervention (no or yes) as the independent variable and one-month CPAP adherence behavior (no or yes) as the dependent variable was analyzed. Effect size 
(phi coefficient) was interpreted using the guidelines of .10 as small effect, .30 as medium effect, and .50 as large effect (Cohen, 1992).

2. What is the effect of the CPAP-SAVER intervention on anxiety (as a background factor)? A mixed between-within subjects ANOVA comparing baseline and onemonth anxiety scores between groups (intervention and control) over time (baseline and one month) was analyzed; the interaction effect between the two independent variables (time* group) and the main effects for each of the independent variables (time and group) were determined. Effect size (partial eta squared) was interpreted using the guidelines of .01 as small effect, .06 as medium effect, and .14 as large effect (Cohen, 1988). Paired samples and/or independent sample t-tests were used to explore the main effects.

3. What is the effect of the CPAP-SAVER intervention on CPAP adherence beliefs? A mixed between-within subjects ANOVA comparing baseline and one-month CPAP adherence beliefs scores between groups (intervention and control) over time (baseline and one month) was analyzed; the interaction effect between the two independent variables (time*group) and the main effects for each of the independent variables (time and group) was determined. Effect size (partial eta squared) was interpreted using the guidelines of .01 as small effect, .06 as medium effect, and .14 as large effect (Cohen, 1988). Paired samples and/or independent sample t-tests were used to explore the main effects.

4. What is the effect of the CPAP-SAVER intervention on CPAP adherence attitude? A mixed between-within subjects ANOVA comparing baseline and one-month CPAP adherence attitude scores between groups (intervention and control) over time 
(baseline and one month) was analyzed; the interaction effect between the two independent variables (time* group) and the main effects for each of the independent variables (time and group) was determined. Effect size (partial eta squared) was interpreted using the guidelines of .01 as small effect, .06 as medium effect, and .14 as large effect (Cohen, 1988). Paired samples and/or independent sample t-tests were used to explore the main effects.

5. What is the effect of the CPAP-SAVER intervention on CPAP adherence subjective norm? A mixed between-within subjects ANOVA comparing baseline and onemonth CPAP adherence subjective norm scores between groups (intervention and control) over time (baseline and one month) was analyzed; the interaction effect between the two independent variables (time*group) and the main effects for each of the independent variables (time and group) was determined. Effect size (partial eta squared) was interpreted using the guidelines of .01 as small effect, .06 as medium effect, and .14 as large effect (Cohen, 1988). Paired samples and/or independent sample t-tests were used to explore the main effects.

6. What is the effect of the CPAP-SAVER intervention on CPAP adherence perceived behavioral control? A mixed between-within subjects ANOVA comparing baseline and one-month CPAP adherence perceived behavioral control scores between groups (intervention and control) over time (baseline and one month) were analyzed; the interaction effect between the two independent variables (time* group) and the main effects for each of the independent variables (time and group) was determined. Effect size (partial eta squared) was interpreted using the guidelines of .01 as small effect, .06 as medium effect, and .14 as large effect (Cohen, 1988). Paired samples and/or 
independent sample t-tests were used to explore the main effects.

7. What is the effect of the CPAP-SAVER intervention on CPAP adherence intention? A mixed between-within subjects ANOVA comparing baseline and one-month CPAP adherence intention scores between groups (intervention and control) over time (baseline and one month) was analyzed; the interaction effect between the two independent variables (time*group) and the main effects for each of the independent variables (time and group) was determined. Effect size (partial eta squared) was interpreted using the guidelines of .01 as small effect, .06 as medium effect, and .14 as large effect (Cohen, 1988). Paired samples and/or independent sample t-tests were used to explore the main effects.

8. Does one-month CPAP adherence attitude, CPAP adherence subjective norm, and/or CPAP adherence perceived behavioral control predict one-month CPAP adherence intention? Standard multiple regression was analyzed to determine if one-month CPAP adherence attitude, CPAP adherence subjective norm, and/or CPAP adherence perceived behavioral control (independent variables) were predictive of one-month CPAP adherence intention (dependent variable). The amount of variance the independent variables explained as a group and the amount of unique variance each independent variable contributed to the model was determined.

9. Does one-month CPAP adherence intention predict one-month CPAP adherence behavior? Logistic regression was analyzed to determine if one-month CPAP adherence intention (independent variable) was predictive of one-month CPAP adherence behavior (dependent variable). The amount of variance in CPAP adherence behavior explained by CPAP adherence intention was also determined. 
If participants were noted to have values that appeared missing at random, their data was included in the analyses. Other missing data were evaluated on a case-by-case basis and excluded from analyses (pairwise). Additional testing included Cronbach alpha coefficients and inter-item correlations on the BAI, ABS, and TPB-based questionnaires to assess internal consistency reliability of the instruments in the study sample.

\section{Human Rights and Ethical Considerations}

The Belmont Report Principles (National Commission for the Protection of Human Subjects of Biomedical and Behavioral Research, 1979), including respect for persons (promoting autonomy, ensuring informed consent, and upholding dignity), beneficence (protecting from harm, maximizing benefit and minimizing risk, and maintaining equipoise), and justice (ensuring fair distribution of benefits and burdens), were upheld throughout the study. Before data collection began, institutional review board approval was obtained (WVU Office of Research Integrity and Compliance Protocol Number 1508800881). Each RT completed research ethics training per the Collaborative Institutional Training Initiative (CITI) protocol; the investigator was current with CITI training. In the event that a participant expressed any physical or emotional distress related to the study or demonstrated a moderate to severe anxiety level (BAI score of 16 to 63), the investigator referred the participant to his/her primary care provider (see Incidental Findings Letter, Appendix H).

\section{Respect for Persons}

The RT obtained informed consent from each participant. The consent session was conducted in a private room. During the consent process, the RT explained the purpose and procedures; potential benefits and risks; confidential nature of the study and how the Health Insurance Portability and Accountability Act (HIPAA) principles would be upheld; data and its 
handling; and the voluntary nature of participation. Participants were given a copy of the consent for their records. Participants were reminded that they could withdraw from the study at any time without any recourse or effect on their CPAP treatment.

\section{Beneficence}

The CPAP-SAVER intervention study was designed to promote benefit to participants in the form of improved CPAP adherence, especially for the intervention group. Direct benefit to participants may have included improved adherence that may impact their OSA disease management, decreased risk of morbidity and mortality, and improved health-related quality of life. Indirect benefit may have included satisfaction in helping others improve adherence to CPAP. No other benefits were anticipated. Some participants may not have benefitted from the study at all.

The investigator anticipated minimal risk to participants in the CPAP-SAVER intervention study. Potential risks involved violation of privacy and confidentiality. The investigator maintained all written, study-related data (consent, demographics, instruments, and OSA/CPAP details) in a locked file cabinet and all electronic, study-related data (Excel and SPSS files) on a password-protected computer; the investigator will maintain and secure this data for three years, at which time she will shred all written documents and erase all electronic documents from her computer. Participants were assigned a code number that was used in place of their name/identifying information; this code number was used in place of the participant's name on the demographics form, instruments, and the Excel and SPSS files. The investigator maintained the master list of participant names and assigned codes on a password-protected computer. The investigator monitored for privacy during education/report card sessions while conducting fidelity checks, used a private phone line and her private office with the door closed 
to conduct the support telephone calls, and discussed with participants the importance of confidentiality. Research assistants were trained per CITI protocol, were trained in research confidentiality per the CPAP-SAVER protocol (see the CPAP-SAVER Intervention Study Protocol Training Manual), and signed a confidentiality agreement (see Appendix I).

\section{Justice}

To the extent possible, the investigator ensured equal distribution of the benefits and burdens of research in the CPAP-SAVER intervention study. However, half of the participants were randomly assigned to the intervention group and may have had additional benefits than the other half of the participants randomly assigned to the control (standard care only) group. All participants were adults aged 18 or older (male and female) who had been diagnosed with OSA and who had been prescribed CPAP for the first time. The investigator anticipated few, minor burdens associated with the CPAP-SAVER intervention study, mainly related to participating in the consent process, completing the demographics survey and instruments, enduring the eight minutes of teaching for the airway/video/education components before the initial CPAP teaching, and actively participating in the telephone conversations during the support/subjective norm calls. The CPAP-SAVER intervention participants did not have to make any additional trips to the home medical supply facility compared to the standard care group.

\section{Methods to Enhance Rigor}

A priori power analysis, randomization, intervention fidelity checks, and reliable instrumentation were implemented to enhance the CPAP-SAVER intervention study rigor. $A$ priori power analysis was conducted with $\mathrm{G}^{*}$ Power 3.1 (Faul et al., 2007). Participants were randomized to either the intervention or control group to improve the chance of homogenous groups and equally-distributed potential confounding variables. The investigator conducted 
extensive training and booster sessions with the research assistants, and supplemented the training with the use of a protocol manual and monthly fidelity checks. The investigator used instruments that had been tested for reliability and validity in other healthcare-related samples.

\section{Advantages and Limitations of the Design}

The advantages of the CPAP-SAVER intervention study design outweighed the limitations. A quantitative research design was used to test hypotheses and, thus, assisted the author in answering the posed research questions related to the CPAP-SAVER intervention and other study variables. Since CPAP adherence is such a major problem in OSA management and nonadherence has myriad sequelae, research to test this intervention was warranted and the results may have a major impact on CPAP adherence in the OSA population. Disadvantages of the design operationalized in the CPAP-SAVER study included overall cost, extensive planning to ensure proper randomization and other aspects of intervention fidelity, and careful statistical analyses and data interpretation. These limitations would not be recognized as such as the intervention is implemented in the healthcare setting. However, testing the intervention in a larger, more diverse sample in a randomized controlled trial will be necessary before the intervention reaches the full implementation stage.

\section{Feasibility of the Proposed CPAP-SAVER Intervention Study}

Participant recruitment and intervention fidelity were the author's greatest concerns related to the feasibility of the CPAP-SAVER intervention study. The author recruited participants from two home medical supply facilities. Since potential participants were most likely coming for help with their OSA because they were having symptoms, the author anticipated that most patients approached about the study would agree to participate; this was augmented by the fact that participants would not have to make additional trips to the home 
medical supply office (above standard care) to participate and the risk/burden associated with the study was expected to be minimal.

The investigator was very cognizant of intervention fidelity. Since the RT were involved in the consent process and the study's implementation, the author carefully and thoroughly trained the research assistants (RT) in the areas of confidentiality, informed consent, and the airway/video/education/report card portions of the intervention. As previously discussed, each RT completed CITI training as required by the IRB. The investigator used a protocol manual throughout the initial training session, the booster session, and the study itself to provide a resource for the RT. The investigator conducted frequent intervention fidelity checks by making visits to the home medical supply facilities, using a checklist to guide the visit (see Appendix F). The investigator encouraged RT to contact her immediately for questions or concerns that arose during the course of the study. In addition, the investigator evaluated each intervention group participant's perception of the effectiveness of the intervention components by conducting and analyzing an Intervention Effectiveness Survey (see Appendix G).

\section{Summary}

This chapter provided an overview of the CPAP-SAVER intervention study method. The research design, sample characteristics, intervention components, instruments, data collection process, data analyses for each research question, and strategies to enhance study rigor were described in detail. Human rights considerations, based on the Belmont Principles of respect for persons, beneficence, and justice, were highlighted. Advantages and limitations of the research design, as well as feasibility concerns related to recruitment and intervention fidelity, were discussed. 


\section{Chapter 4: Results}

Seventy-four individuals met the inclusion and exclusion criteria for the CPAP-SAVER study, however, eight individuals declined participation (did not want to participate in a research study). A total of 66 participants were recruited from two facilities (Site A, $n=64$; Site B, $n=2$ ) over ten months, with 33 participants randomly assigned to each group (intervention and standard care). One intervention group participant was lost to attrition midway through the study. Missing data were determined to be missing at random and were included in the statistical analyses; where applicable, cases were excluded pairwise.

\section{Demographics}

Univariate analyses (frequencies and descriptives) for the entire sample are reported in Tables 6 through 9. The sample was comprised of mainly white (97\%), middle-aged ( $M=51.8$, $S D=13.1$ years) men $(54.5 \%)$. The majority of participants had a college or technical school background (62.1\%), reported an annual household income of higher than $\$ 40,000$ (70.3\%), were married (66.7\%), and had a bed partner (63.1\%). Most participants were classified as obese, with a mean BMI of $35.7 \mathrm{~kg} / \mathrm{m}^{2}$. Participants reported hypertension $(62.1 \%)$ as the most common comorbidity.

Most participants were diagnosed with moderate to severe OSA (56.1\%), with a mean AHI of $26.2(S D=22.1)$ at the time of their sleep study, and were prescribed CPAP at a mean pressure of 9.3 centimeters of water $(S D=2.6)$. The most common CPAP machine used by participants was the Philips Respironics DreamStation (78.8\%), set at 2 centimeters of water expiratory pressure relief (69.7\%). Most participants wore a full-face mask initially $(62.1 \%)$ and throughout the month of the study (59.1\%). Within one week of CPAP use, the mean participant AHI decreased to $5.0(S D=6.7)$. Most participants used CPAP at the level of adherence (at least 
four hours per night for $70 \%$ of the time) at one week $(75.8 \%)$ and one month $(72.7 \%)$.

Participants used CPAP a mean of 36.3 hours $(S D=15.6)$ during the first week and a mean of 157.6 hours $(S D=66.0)$ during the first month, for an average of 5.4 hours a night.

Univariate analyses (frequencies and descriptives) for the sample, by group (intervention versus standard care), are reported in Tables 10 through 14. After comparing the frequencies (chi-square tests) and descriptives (independent samples t-tests) analyses by group, the groups were determined to be homogeneous. There were no statistically significant differences between the intervention and standard care groups as to general and sleep demographics.

Univariate analyses were also compared by gender and demonstrated that males in the study had higher means for both one-week and one-month CPAP use in hours, hours per night worn, and number of nights worn (see Table 15); however, independent samples t-tests comparing the variable means by gender did not indicate statistical significance. Eighty-percent of females (24/30) met the definition of CPAP adherence at one week, compared to $72 \%$ of males (26/36); only 67\% of females (20/30) met the CPAP adherence definition at one month, compared to $78 \%$ of males (28/36). Chi-square tests comparing gender and meets CPAP adherence definition (yes or no) for both one week and one month demonstrated no statistical significance. Independent samples t-tests comparing instrument scores by gender revealed differences in the BAI scores at baseline $(t[64]=2.52, p=.014)$ and at one month $(t[62]=2.33$, $p=.023)$; males had lower mean BAI scores at both times $(9.0 \pm 9.3$ at baseline and $3.7 \pm 5.6$ at one month) compared to females (14.6 \pm 8.6 at baseline and $7.9 \pm 8.8$ at one month). Even though women had higher mean BAI scores than men at both times, the anxiety levels represented by the scores were minimal to mild for both genders. 


\section{Scale Reliability in the CPAP-SAVER Study}

Reliability testing was performed on the instruments used in the study. The Cronbach alpha coefficients for the baseline and one-month BAI were .89 and .92 , respectively. Reliability testing of the Apnea Beliefs Scale demonstrated Cronbach alpha coefficients of .82 (baseline) and .88 (one month). For the Theory of Planned Behavior subscores (comprised of only three or four items), the baseline and one-month mean inter-item correlation values were:

Attitude: .42 and .44

Subjective Norm: .14 and .24

Perceived Behavioral Control: .06 and .02

Intention: .75 and .50

The Cronbach alpha coefficients for the baseline and one-month Theory of Planned Behavior total scores were .76 and .78 , respectively.

\section{Assumptions Testing}

Statistical analyses did not reveal any major assumptions violations for homogeneity of intercorrelations, homogeneity of variance, multicollinearity, singularity, outliers, normality, linearity, homoscedasticity, or independence of residuals. Histograms, scatterplots, P-P plots, QQ plots, boxplots, correlations, and cell frequency were examined. In addition, collinearity diagnostics (tolerance and variance inflation ratio), Mahalanobis Distances, Cook's Distances, Box's M statistics, and Levene's Test of Equality of Error Variances were also analyzed for the assumptions testing and did not reveal any violations.

\section{Research Question 1: Effect of the Intervention on CPAP Adherence Behavior}

A chi-square test for independence (with Yates' Continuity Correction) was analyzed to compare one-month CPAP adherence of the two groups (intervention and standard care) and indicated no significant association, $\chi^{2}(1, N=66)=.08, p=.778$, phi $=-.07$ (very small effect). Paired samples t-tests for the intervention $(t[32]=-13.69, p=.001)$ and standard care $(t[32]=-$ 
$11.69, p=.001)$ groups revealed significant increases in CPAP use from one week to one month, however, an independent samples t-test did not reveal a significant difference in the group means for CPAP use in hours at one week $(t[64]=.83$, mean difference $=3.21, p=.408)$ or one month $(t[64]=.43$, mean difference $=6.97, p=.672)$. Intervention group participants used CPAP for a total of 5086.60 hours over one month, compared to standard care participants' total use of 5316.46 hours.

Since most of the intervention was implemented during the first week of CPAP use, the author was interested in CPAP performance during that time. Intervention participants used their CPAP an average of $37.9 \pm 14.6$ hours over $6.3 \pm 1.1$ nights during the first week, compared to the standard care group participants' mean use of $34.7 \pm 16.6$ hours over $5.9 \pm 2.0$ nights. Intervention participants used their CPAP an average of 5.6 \pm 2.0 hours per night during the first week; standard care participants used theirs an average of $5.2 \pm 2.4$ hours per night. Independent samples t-tests revealed no statistically significant differences in the group means for one-week CPAP use, average hours per night, or number of nights worn. The author also examined total CPAP use for both groups; the intervention group used CPAP a total of 1251.91 hours during the first week, compared to 1146.00 hours by the standard care group, for a difference of 105.91 hours more CPAP use by the intervention group in the first week.

\section{Research Question 2: Effect of the Intervention on Anxiety (as a Background Factor)}

A mixed between-within ANOVA was conducted to assess the impact of group (intervention versus standard care) on participants' anxiety level (BAI score over time [baseline and one month]). There was no significant interaction between group and time, Wilks' Lambda $=.99, F(1,62)=.74, p=.392$, partial eta squared $=.01$ (small effect). There was, however, a substantial main effect for time, Wilks' Lambda $=.68, F(1,62)=28.87, p=.001$, partial eta 
squared $=.32$ (very large effect), demonstrating a significant within-subjects reduction in BAI scores from baseline to one month (see Table 14 for BAI group means; intervention mean difference $=5.1 \pm 8.3$ and standard care mean difference $=7.0 \pm 9.6$ ). The one-month BAI mean score was lower for the intervention group $(5.0 \pm 7.3)$ than the standard care group $(6.1 \pm 7.5)$, and indicated a lower level of anxiety for the intervention group. To further examine the main effect of time, paired samples t-tests were analyzed; the results supported the ANOVA main effect of time for the intervention $(t[30]=3.40, p=.002)$ and standard care $(t[32]=4.20, p=$ .001) groups. The main effect of group (between-subjects effect) on anxiety was not significant $(F[1,62]=1.23, p=.273$, partial eta squared $=.02$ [small effect] $)$. [Note: Nineteen participants in the study had baseline and/or one-month BAI scores ranging from 16 to 63, which indicated a moderate to severe level of anxiety; these participants were mailed Incidental Findings Letters (see Appendix H).]

\section{Research Question 3: Effect of the Intervention on CPAP Adherence Beliefs}

A mixed between-within ANOVA was conducted to determine the impact of group (intervention versus standard care) on participants' CPAP adherence beliefs (Apnea Beliefs Scale scores over time [baseline and one month]). There was no significant interaction between group and time (Wilks' Lambda $=.96, F[1,62]=2.46, p=.122$, partial eta squared $=.04$ [small effect]). In addition, the analysis indicated that there was not a main effect for time, Wilks' Lambda $=.99, F(1,62)=.40, p=.531$, partial eta squared $=.01($ small effect $)$. However, the results for the CPAP adherence beliefs between groups approached significance (alpha set at .05; $p=.057), F[1,62]=3.77, p=.057$, partial eta squared $=.06$ [medium effect] $)$. For the entire sample, the mean Apnea Beliefs Scale score was $89.64 \pm 10.33$ (range $54-114$ ) at baseline and $90.64 \pm 12.59$ (range $62-115$ ) at one month. By group, the mean scores at baseline were $90.5 \pm$ 
6.7 (range $76-107$ ) for the intervention group and $88.7 \pm 13.0$ (range $54-114$ ) for the standard care group; the mean scores at one month were $94.3 \pm 12.2$ (range 66 - 115) for the intervention group and $87.2 \pm 12.1$ (range $62-108$ ) for the standard care group. To further examine the main effect of group, an independent samples t-test comparing group mean CPAP adherence beliefs scores revealed a significant difference in the mean one-month CPAP adherence beliefs scores between the groups $(t[62]=-2.33, p=.023)$; the intervention group had significantly higher mean CPAP adherence beliefs scores than the standard care group at one month. Based on interpretation guidelines by Smith et al. (2004a), the results indicated that the CPAP-SAVER intervention group had more positive attitudes and beliefs about CPAP adherence than the standard care group at one month. Since an independent samples t-test demonstrated that the groups did not differ at baseline on their mean ABS scores $(t[64]=-.712, p=.480)$ but did at one month, the CPAP-SAVER intervention may have contributed to the development of more positive beliefs about CPAP adherence in the intervention group.

\section{Research Question 4: Effect of the Intervention on CPAP Adherence Attitude}

A mixed between-within ANOVA was conducted to assess the impact of group (intervention versus standard care) on participants' CPAP adherence attitude scores over time (baseline and one month). There was no significant interaction between group and time, Wilks' Lambda $=.96, F(1,62)=2.83, p=.097$, partial eta squared $=.04$ (small effect). The analysis also indicated that there was not a main effect for time, Wilks' Lambda $=.96, F(1,62)=2.39$, $p=.127$, partial eta squared $=.04$ (small effect) or group $(F[1,62]=1.85, p=.179$, partial eta squared $=.03$ [small effect]). Although mean CPAP adherence attitude scores at one month were higher for the intervention group $(M=6.1)$ than the standard care group $(M=5.6)$, the ANOVA revealed no statistically significant differences between or within the groups. 


\section{Research Question 5: Effect of the Intervention on CPAP Adherence Subjective Norm}

A mixed between-within ANOVA was conducted to assess the impact of group (intervention versus standard care) on participants' CPAP adherence subjective norm scores over time (baseline and one month). There was no significant interaction between group and time, Wilks' Lambda $=.99, F(1,63)=.13, p=.716$, partial eta squared $=.002$ (very small effect). The analysis also indicated that there was not a main effect for time, Wilks' Lambda $=.99, F(1,63)=$ $.74, p=.393$, partial eta squared $=.01$ (small effect) or group $(F[1,63]=.38, p=.540$, partial eta squared $=.01$ [small effect]). Although mean subjective norm scores increased over time for both the intervention (baseline $M=5.38$, one month $M=5.45$ ) and standard care (baseline $M=$ 5.43, one month $M=5.61$ ) groups, the ANOVA results revealed no statistically significant differences between or within the groups.

\section{Research Question 6: Effect of the Intervention on CPAP Adherence PBC}

A mixed between-within ANOVA was conducted to assess the impact of group (intervention versus standard care) on participants' CPAP adherence perceived behavioral control scores over time (baseline and one month). There was no significant interaction between group and time, Wilks' Lambda $=.99, F(1,61)=.03, p=.855$, partial eta squared $=.001$ (very small effect). The analysis also indicated that there was not a main effect for time, Wilks' Lambda $=.99, F(1,61)=.42, p=.520$, partial eta squared $=.01$ (small effect $)$ or group $(F[1,61]$ $=.01, p=.910$, partial eta squared $=.001$ [very small effect] $).$ Although perceived behavioral control scores decreased slightly over time for both the intervention (baseline $M=4.94$, one month $M=4.84$ ) and standard care (baseline $M=4.90$, one month $M=4.84$ ) groups, the decrease was not significant. 


\section{Research Question 7: Effect of the Intervention on CPAP Adherence Intention}

A mixed between-within ANOVA was conducted to assess the impact of group (intervention versus standard care) on participants' CPAP adherence intention scores over time (baseline and one month). There was no significant interaction between group and time, Wilks' Lambda $=.98, F(1,62)=1.33, p=.254$, partial eta squared $=.02$ (small effect). There was, however, a significant main effect for time, Wilks' Lambda $=.88, F(1,62)=8.18, p=.006$, partial eta squared $=.12$ (medium effect), demonstrating a within-subjects reduction in CPAP adherence intention scores from baseline (intervention $M=6.6$, standard care $M=6.7$ ) to one month (intervention $M=6.3$, standard care $M=6.1$ ). To further examine the main effect of time, paired samples t-tests conducted for each group showed that there was a significant decrease in the intention scores from baseline to one month for the standard care group $(t[32]=$ $2.63, p=.013)$, but not for the intervention group $(t[30]=1.34, p=.190)$. The one-month intention scores were higher for the intervention group $(6.3 \pm 1.1)$ than the standard care group (6.1 \pm 1.1$)$, however, the main effect of group was not significant $(F[1,62]=.31, p=.579$, partial eta squared $=.01[$ small effect] $)$.

\section{Research Question 8: Prediction of CPAP Adherence Intention}

Standard multiple regression was analyzed to determine if one-month CPAP adherence attitude, CPAP adherence subjective norm, and/or CPAP adherence perceived behavioral control were predictive of one-month CPAP adherence intention. This model explained $52.1 \%$ of the variance in CPAP adherence intention scores (ANOVA $F[3,64]=22.12, p=.001$ ). All three variables - CPAP adherence attitude (standardized beta $=.35, p=.001$ ), CPAP adherence subjective norm (standardized beta $=.36, p=.001$ ), and CPAP adherence perceived behavioral control (standardized beta $=.24, p=.018$ ) - were significant independent predictors of CPAP 
adherence intention scores. CPAP adherence subjective norm demonstrated the strongest unique contribution to CPAP adherence intention scores, explaining $10.4 \%$ of the variance; CPAP adherence attitude and CPAP adherence perceived behavioral control uniquely explained 9.7\% and $4.7 \%$, respectively, of the variance in CPAP adherence intention scores.

\section{Research Question 9: Prediction of CPAP Adherence Behavior}

Direct logistic regression was performed to assess the impact of one-month CPAP adherence intention on one-month CPAP adherence behavior. The full model was statistically significant $\left(\chi^{2}(1, N=65)=9.93, p=.002\right)$ and indicated that the model was able to distinguish between participants who were adherent or not. The model as a whole explained between $14.1 \%$ (Cox and Snell R square) and 21.0\% (Nagelkerke R square) of the variance in CPAP adherence behavior, and correctly classified $78.5 \%$ of cases. The odds of a person demonstrating onemonth CPAP adherence behavior was 2.30 times higher for a participant with a higher onemonth CPAP adherence intention (95\% CI 1.32, 4.02) than for a participant with a lower onemonth CPAP adherence intention $(p=.003)$. The model demonstrated a $93.9 \%$ sensitivity and 31.3 specificity. Of the participants predicted to be CPAP adherent, the model accurately picked $80.7 \%$ of them (positive predictive value); of those predicted to not be CPAP adherent, the model accurately picked $62.5 \%$ of them (negative predictive value).

\section{Support Calls}

The most troublesome OSA symptom reported by intervention participants was excessive sleepiness $(15 / 33,45.5 \%)$. Gasping/choking at night $(6 / 33,18.2 \%)$, snoring $(4 / 33,12.1 \%)$, nonrefreshing sleep $(4 / 33,12.1 \%)$, sleep fragmentation $(3 / 33,9.1 \%)$, and morning headaches $(1 / 33,3.0 \%)$ were also reported. During the first support call (mid-week one, after four nights of CPAP use), the majority $(27 / 33,81.8 \%)$ reported improvement in this symptom since starting 
their CPAP use. The majority of the intervention participants also reported that they believed it was important to wear their CPAP every night $(33 / 33,100 \%)$, that their family/people closest to them believed it was important for them to wear their CPAP every night $(32 / 33,97.0 \%)$, that their family/people closest to them were supportive of their decision to treat their OSA (33/33, $100 \%$ ), and that their family/people closest to them were supportive of their decision to wear CPAP (32/33, 97\%). Similar numbers were reported during the second support call (mid-week two, after 11 nights of CPAP use).

During the support calls to intervention participants, a question about machine/mask problems they were having was posed. Machine and mask problems reported by the intervention group participants were few and were easily resolved by the RT. The machine-related problems were dryness of the nose and throat $(n=8)$, machine malfunction problems $(n=2)$, and adjusting to tubing positioning when sleeping $(n=2)$. Mask problems reported included mask leakage $(n$ $=5$ ), mask difficulties (rubbing, $n=2$; size too large, $n=1$; and smell, $n=1$ ), seal/pressure blowing cheeks out $(n=2)$, and claustrophobia $(n=1)$. When these problems were reported, the author encouraged the participant to contact the RT for troubleshooting suggestions. During the second support call, most participants reported that they had contacted the RT and that the problem had been resolved, usually by adjusting the machine's humidity settings, trading out the machine, using a different pillow to sleep, and/or mask adjustments. Neither machine nor mask problems delayed the start or prolonged the duration of the participants' involvement in the study. One participant in the intervention group delayed starting her CPAP use due to personal issues; she was in the study one week longer as a result.

\section{Report Card Grades}

Intervention participants and the research assistants (respiratory therapists) reported 
grades for the participants' one-week and one-month CPAP use (see frequencies, Table 17). The mean one-week report card grades by participants and research assistants were 3.20 (B) and 2.91 (C), respectively $(r=.75, p=.001)$; the mean one-month report card grades by participants and research assistants were lower at 2.97 (grade of C) and 2.73 (grade of C), respectively ( $r=.89, p$ $=.001)$. Paired samples t-tests comparing the means of the report card grades by participants and research assistants were not significant at one week $(t[32]=1.97, p=.057)$ or one month $(t[32]=1.85, p=.073)$.

\section{Intervention Effectiveness Survey}

The CPAP-SAVER intervention Effectiveness Survey was completed by each intervention participant (see Appendix G). The survey analyses are presented in Table 18. The majority of participants rated each component as somewhat or extremely ( 3 or 4 on the 0 to 4 Likert scale) as to whether the intervention was helpful, liked, understood, and motivating. Based on the mean scores for the CPAP-SAVER components, intervention participants reported that the airway model was the most helpful component; the support calls and report card were the most liked component; and the support calls and the airway model were the most understood components. In addition, participants reported that the support calls, airway model, video, and report card motivated their CPAP use.

\section{Post Hoc Power Analysis}

Using G*Power 3.1 (Faul et al., 2007), a post hoc power analysis was conducted. The analysis revealed that the study was underpowered. Based on the one-month adherence of the intervention group $(n=33$, adherence $=70 \%)$ and the one-month adherence of the standard care group $(n=33$, adherence $=76 \%)$, the power was determined to be 0.14 . 


\section{Summary}

This chapter was a presentation of the results of the CPAP-SAVER Intervention Study statistical analyses. Demographics, including univariate and bivariate analyses, were presented for the entire sample and by group (intervention versus standard care), and were displayed in tables and reported in text. Scale reliabilities were reported for each instrument used in the study. Results of assumptions testing and inferential statistical analyses for each research question, including chi-square, t-tests, ANOVAs, and regressions, were emphasized. In addition, results of statistical analyses conducted on data collected from the support calls, report cards, and CPAP-SAVER Intervention Effectiveness Surveys were described. A post hoc power analysis was reported. 


\section{Chapter 5: Discussion}

Noncompliance is two people working toward different goals.

(Rakel \& Jonas, 2016, p. 20)

OSA affects at least 25 million adults in the United States (American Academy of Sleep Medicine, 2014). CPAP is the gold standard treatment, however adherence is poor $(30-60 \%$; Weaver \& Sawyer, 2010). CPAP nonadherence contributes to myriad comorbidities that affect OSA patients' healthcare utilization and quality of life. Comorbidities associated with CPAP nonadherence, including cardiovascular disease, cerebrovascular disease, and diabetes (Glebocka et al., 2006; Pack, 2006; Rakel, 2009), place tremendous physical and financial burdens on the healthcare system (Tarasiuk \& Reuveni, 2013). CPAP nonadherence impacts ability to function, potentially leading to traffic, work-related, and domestic accidents (Akashiba et al., 2002). These factors may have a negative impact on overall quality of life, especially related to interpersonal problems within partner, family, social, and work relationships (Reishtein et al., 2006). Treatment with CPAP has been shown to reverse the trend of increasing healthcare utilization by OSA patients and provide long-term health benefits (Tarasiuk \& Reuveni, 2013). A theory-based, multidimensional intervention designed to improve CPAP adherence was developed and tested.

\section{Discussion of the Results}

A theory-based intervention, referred to as CPAP-SAVER, was implemented for this dissertation study. The overall purpose of the study was to examine the effect of the CPAPSAVER intervention on CPAP adherence among adults with newly diagnosed OSA. The intervention was based on the constructs of the Theory of Planned Behavior (Ajzen, 1985, 1991). Nine research questions were posed to explicate the full effect of the intervention on the constructs - anxiety (as a background factor), CPAP adherence beliefs, CPAP adherence 
attitude, CPAP adherence subjective norm, CPAP adherence perceived behavioral control, CPAP adherence intention, and CPAP adherence behavior.

\section{Summary of Results}

- Research question 1: Effect of the CPAP-SAVER intervention on one-month CPAP adherence

○ There was no statistically significant difference between groups as to one-month CPAP adherence.

- Research question 2: Effect of the CPAP-SAVER intervention on anxiety (as a background factor)

- There was no statistically significant interaction effect.

- There was a statistically significant effect of time (BAI scores decreased over time).

- There was no statistically significant effect of group.

- Research question 3: Effect of the CPAP-SAVER intervention on CPAP adherence beliefs

- There was no statistically significant interaction effect.

- There was no statistically significant effect of time.

- There was no statistically significant effect of group, but almost reached significance (alpha set at .05; $p=.057$ ).

- Research question 4: Effect of the CPAP-SAVER intervention on CPAP adherence attitude

- There was no statistically significant interaction effect.

- There was no statistically significant effect of time.

- There was no statistically significant effect of group.

- Research question 5: Effect of the CPAP-SAVER intervention on CPAP adherence subjective norm

○ There was no statistically significant interaction effect.

- There was no statistically significant effect of time.

- There was no statistically significant effect of group.

- Research question 6: Effect of the CPAP-SAVER intervention on CPAP adherence perceived behavioral control

- There was no statistically significant interaction effect.

- There was no statistically significant effect of time.

- There was no statistically significant effect of group.

- Research question 7: Effect of the CPAP-SAVER intervention on CPAP adherence intention

- There was no statistically significant interaction effect.

- There was a statistically significant effect of time (intention scores decreased over time).

- There was no statistically significant effect of group. 
- Research question 8: One-month CPAP adherence attitude, subjective norm, and/or perceived behavioral control predictive of one-month CPAP adherence intention

○ The model statistically significantly explained $52.1 \%$ of variance in CPAP adherence intention.

○ CPAP adherence attitude (9.7\%), subjective norm (10.4\%), and perceived behavioral control $(4.7 \%)$ made statistically significant, unique contributions to the variance in CPAP adherence intention.

- Research question 9: One-month CPAP adherence intention predictive of one-month CPAP adherence behavior

○ The model statistically significantly explained between $14.1 \%$ and $21.0 \%$ of the variance in CPAP adherence behavior.

○ Sensitivity was $93.9 \%$, specificity was $31.3 \%$, positive predictive value was $80.7 \%$, and negative predictive value was $62.5 \%$.

\section{Theoretical Framework: The Theory of Planned Behavior}

The Theory of Planned Behavior (Ajzen, 2011) postulates that personal, social, environmental, and internal factors guide the process of behavioral intention and subsequent action. Ajzen $(1985,1991)$ identified constructs, including background factors, beliefs, attitude, subjective norm, and perceived behavioral control, that tend to influence intention and eventual behavior. The Theory of Planned Behavior has been used as a framework and tested in various health-related intervention studies and was shown to significantly predict intention and behavior. Based on the author's literature review, this was the first study to apply its constructs in the OSA-CPAP population.

\section{Results in Context of the Theoretical Constructs and Previous Work}

\section{Anxiety as a Background Factor}

In previous studies, anxiety was found to be common among individuals with OSA (Saunamaki \& Jehkonen, 2007; Shapiro, 2014). OSA symptoms, including nonrefreshing sleep and awakenings due to apneic episodes, were shown to be predictors of anxiety in a populationbased study (Shapiro et al., 2014). In work by Kjelsberg et al. (2005), anxiety was shown to be a 
predictor of CPAP nonadherence. Anxiety has also been shown to affect quality of life and physiological status among individuals with OSA (Ye, Liang, \& Weaver, 2008).

In the CPAP-SAVER intervention study, anxiety levels significantly decreased over time. The intervention group $(M=5.0)$ demonstrated lower one-month BAI scores than the standard care group $(M=6.1)$, which indicated a lower mean level of anxiety one-month post CPAP use; however, anxiety level was not statistically significant by group (intervention or standard care). Men in the study had significantly lower anxiety levels compared to women at both baseline and one month; this is consistent with the literature and believed to be due to a higher emotional response and perceived threat of OSA by women (Sampaio, Pereira, \& Winck, 2012; Sanford et al., 2008). However, men may not be as open with feelings of anxiety or as apt to report them (Simon \& Nath, 2004).

Screening for anxiety in individuals diagnosed with OSA and prescribed CPAP is recommended. Since previous studies have shown that anxiety may impact CPAP adherence, lower anxiety scores throughout CPAP use may affect long-term CPAP adherence and subsequent quality of life for individuals with OSA. Focused and individualized support early in the CPAP acclimation period, especially over the first few weeks, may be necessary to maintain a decreased anxiety level.

\section{CPAP Adherence Beliefs}

OSA- and CPAP-related beliefs have been shown to be predictive of CPAP adherence in adult samples (Poulet et al., 2009; Smith et al., 2004a; Smith et al., 2004b). In a study by Poulet et al. (2009), individuals with maladaptive beliefs (ABS score less than 84.5 out of 120) were 2.21 times more likely to be nonadherent to $\mathrm{CPAP}(\mathrm{CI}=1.03,4.72, p=.04)$. In addition, OSA- 
and CPAP-related beliefs were shown to trend upward over time, even as far as three months post CPAP use (Smith et al., 2004b).

In the current study, mixed between-within ANOVA testing approached significance for the effect of group on one-month apnea beliefs $(p=.057)$, and indicated that the intervention group had a higher mean one-month ABS score $(M=94.3, S D=12.2)$ compared to the standard care group $(M=87.2, S D=12.1)$. According to Smith et al. (2004a), higher ABS scores indicate more positive attitudes and beliefs about CPAP adherence. Independent samples t-testing in the CPAP-SAVER intervention study indicated that the intervention and standard care groups did not significantly differ at baseline $(p=.480)$, but did at one month $(p=.023)$. The CPAPSAVER intervention may have had an impact on the development of more positive CPAPadherence beliefs over the first month of CPAP use.

Beliefs about CPAP adherence have been shown to influence CPAP use (Poulet et al., 2009). Positive beliefs may be important in improving and/or sustaining CPAP adherence over time, especially in the development of long-term adherence. Promoting positive beliefs consistently in the early CPAP-treatment process, especially by healthcare providers and significant others, may influence this. Assessing OSA-CPAP beliefs early in the treatment trajectory may provide insight as to patients at risk for CPAP nonadherence.

\section{CPAP Adherence Attitude}

Attitude, as defined by Ajzen (2005), is the favorable (positive) or unfavorable (negative) appraisal of performing the behavior in question - in this study, CPAP adherence. Attitude is determined by beliefs and consequences of behavior, in addition to the person's evaluation of the outcomes associated with the behavior. In the Theory of Planned Behavior, it is proposed that an 
individual will be more apt to perform a behavior if he/she expects positive outcomes to result from the behavior; this, then promotes a more favorable attitude toward the behavior.

In the CPAP-SAVER study, the groups did not significantly differ over time as to attitude; the overall mean score was 5.6 out of 7 at baseline for both groups. However, at one month, attitude scores were higher for the intervention group $(M=6.1, S D=1.0)$ compared to the standard care group $(M=5.6, S D=1.0)$. The components of the CPAP-SAVER intervention designed to impact attitude, including the airway model, video, and education sheet, may have contributed to the difference in the mean attitude scores; this is also evident in the mean scores from the Intervention Effectiveness Survey - the airway model received the highest overall mean for helpful (3.6 out of 4), understood (3.8 out of 4), and motivated (3.2 out of 4). It is possible that the airway model provided a visual representation to which intervention participants could relate and which affected their responses (cognitive, affective, and conative) toward the CPAP and the effects if could have on their airway. The video also received a high mean score for motivated (3.2 out of 4); this component may have also promoted a more favorable attitude toward CPAP adherence, especially since the video portrays an OSA airway without CPAP and then with CPAP. Since intervention participants rated the airway model and video high as to components they found helpful and motivating, these two components may have promoted a sense of a more favorable outcome and, therefore, a more positive attitude and intention toward CPAP adherence.

\section{CPAP Adherence Subjective Norm}

Subjective norm is the perceived pressure to perform or not perform a behavior (Ajzen, 2005). It is influenced by specific individuals or groups, including parents, spouse/significant other, close friends, coworkers, and experts. According to the Theory of Planned Behavior, a 
person who believes that most referents think he/she should perform the behavior will perceive social pressure to do so.

In this study, the support calls were designed to promote a subjective norm toward CPAP adherence. ANOVA testing revealed that there was not a significant effect by group over time; scores for both groups increased over time from baseline to one month - intervention group, from 5.38 to 5.45 ; and standard care group from 5.43 to 5.61. Support calls were made to intervention participants at mid-week one (after four nights of CPAP use) and at mid-week two (after 11 nights of CPAP use). Intervention participants reported that they liked the support calls and many expressed disappointment to the author when they were told that the second call was the last call. However, it is possible that the intervention dosage strength, timing, and/or frequency of the calls affected the subjective norm. As far as dosage strength, more pressure to be CPAP adherent and more emphasis on the effects of not being adherent may have helped improve the effect of the support calls in promoting a stronger subjective norm.

\section{CPAP Adherence Perceived Behavioral Control}

The perception of behavioral control involves self-efficacy and controllability (Ajzen, 2005). According to the Theory of Planned Behavior, behavior is strongly influenced by external factors, such as powerful others or chance, and internal factors, such as the person's confidence in his/her ability to perform the behavior. An individual's perception of behavioral control can influence his/her behavior indirectly (through intention) or directly (Ajzen, 2005), and may be impacted by resources, opportunities, and obstacles.

Perceived behavioral control scores for both groups in the CPAP-SAVER study decreased over time, although not significantly (intervention group baseline $M=4.94$, one month $M=4.84$; standard care group baseline $M=4.90$, one month $M=4.84$ ). Because the scores 
decreased, this indicated that participants may have perceived less behavioral control (lower selfefficacy and controllability) from baseline to one month. There are many explanations for this the first month of CPAP use can be challenging, as the individual acclimates to the routine of CPAP use, finding the most comfortable mask, adapting to sleep with a mask on the face, and maintaining the CPAP machine. In the CPAP-SAVER study, the component specifically designed to promote a stronger perception of behavioral control was the report card. Intervention participants reported that they liked the report card (3.4 out of 4), that they understood it (3.6 out of 4), and that they were motivated by it (3.2 out of 4). However, work to improve its efficacy may be needed.

As with the support calls, it is possible that the intervention dosage strength, timing, and/or frequency related to the report card were not enough to impact the participant's perception of behavioral control. There have been no previous studies examining the use of a report card for CPAP use, but there have been studies where participants were shown other objective data, such as their sleep study results (Falcone et al., 2014) and baseline OSA data (Roecklin et al., 2010). Since report cards have been used successfully in patients with diabetes ("Physician group," 2003), on dialysis (Karalis, 2001), and recent stroke (UCLA, 2000), there may be advantages of its use in the OSA-CPAP population. Further research to explore the dosing and timing of the report card, perhaps a weekly review of it in the first month of use, may be helpful. One CPAP-SAVER intervention participant described the use of a free phone application he used to review his nightly AHI and other CPAP data; this technology may also be an effective resource in promoting a stronger perception of confidence, self-efficacy, and control.

\section{CPAP Adherence Intention}

Ajzen (1991) described intention as how hard a person is willing to try - how much of an 
effort he/she is planning to exert - in order to perform the specified behavior. The stronger the intention to engage in a particular behavior, the more likely should be its performance. Based on the Theory of Planned Behavior's premises, one's intention can be predicted with considerable accuracy by measuring the preceding constructs - attitude, subjective norm, and perceived behavioral control.

In the CPAP-SAVER study, participants' intention scores decreased in both groups over time. The intervention group had a mean intention score of 6.6 at baseline and 6.3 at one month; the standard care group had a mean intention score of 6.7 at baseline and 6.1 at one month. There was less of a decline in the intervention group scores from baseline to one month (mean difference of 0.3 compared to 0.6 ), and a higher mean intention score at one month compared to the standard care group. Since both groups had high levels of intention at baseline, there may have been somewhat of a ceiling effect for this construct. In addition, since perceived behavioral control scores decreased from baseline to one month for both groups, this may have had an impact on the intention toward CPAP adherence. It is possible that by strengthening an individual's attitude, sense of subjective norm, and perception of behavioral control, an increase in intention to adhere to CPAP may result. Since patients may begin CPAP use with high intention to adhere, continued support throughout the first month of acclimation may be necessary to sustain this intent to adhere.

It should be noted that there may have been selection bias in the convenience sample of this study. The entire group had high intention scores going into the study (at baseline). This strong intention may have influenced the decision to choose to volunteer in a study about the effect of an intervention on adherence. In addition, $62 \%$ of the participants had a college or technical school background. 


\section{CPAP Adherence Behavior}

Behavior is the observable response in a given situation, defined by four elements: action (the behavior itself), target (source to which the action is directed), context (circumstance in which it is performed), and time (when it is expected to occur; Ajzen, 2006). The Theory of Planned Behavior proposes that an individual's attitude, subjective norm, and perceived behavioral control produce a relative behavioral intention, and result in actual behavior (Ajzen, 2006). The theory assumes that human behavior is reasoned or planned.

In the CPAP-SAVER intervention study, CPAP adherence behavior was defined based on the current guidelines proposed by the Centers for Medicare and Medicaid Services (2013) wearing CPAP at the prescribed pressure for at least four hours per night over $70 \%$ of the nights. CPAP adherence was used as the dependent variable; this is more stringent than most of the theory-based studies reviewed for this dissertation, where CPAP use in hours was used. Although chi-square testing revealed no significant difference in CPAP adherence (yes or no) between the intervention and standard care groups $(p=.778)$ in the CPAP-SAVER study, it was noted that CPAP use increased significantly for each group from baseline to one month $(p=.001$ for each group). This could be simply due to the passage of time and what would be expected, however, replication of the study in a larger, more diverse sample may clarify this effect.

The percentage of sample participants (collectively) who met the adherence definition was $75.8 \%$ at one week and $72.7 \%$ at one month; these numbers were well above the reported adherence rates of $30 \%$ to $60 \%$. In addition, the entire sample's mean CPAP use per night was 5.4 hours worn over a mean of 6.1 nights at one week and 26.8 nights at one month. More intervention participants met the definition of adherence at one week (78.8\%) than standard care participants $(72.7 \%)$ and wore their CPAP more hours in the first week $(M=37.9)$ than the 
standard care participants $(M=34.7)$. In addition, intervention participants $(M=5.6)$ wore their CPAP more hours per night than standard care participants $(M=5.2)$ in the first week and more nights $(M=6.3)$ than standard care participants $(M=5.9)$ in the first week. Since most of the CPAP-SAVER intervention was implemented within the first week, the increased use and adherence by the intervention group may have been due to effects of the intervention.

Even though intervention group participants wore their CPAP for a total of 105.91 hours more within the first week than the standard care group participants, they did not sustain this increased use. The trend reversed somewhat at one month, with the number of adherent intervention group participants dropping to $69.7 \%$ and the number of adherent standard care group participants increasing to $75.8 \%$. However, intervention group participants wore their CPAP more nights over one month $(M=27.1)$ than standard care participants $(M=26.6)$. Strengthening the constructs (CPAP attitude, subjective norm, and perceived behavioral control), as previously discussed, may be necessary to promote improved CPAP adherence intention and subsequent behavior.

\section{Prediction of CPAP Adherence Intention}

The Theory of Planned Behavior proposes that attitude, subjective norm, and perceived behavioral control predict an individual's intention toward a particular behavior. In fact, Ajzen (2011) suggests that the constructs predict intention with substantial accuracy. In previous healthcare-related studies based on the theory, the constructs (attitude, subjective norm, and perceived behavioral control) predicted anywhere from $14 \%$ to $55.1 \%$ of the variance in intention.

In the regression model for the current study, the CPAP adherence attitude, subjective norm, and perceived behavioral control constructs explained a significant amount of variance in 
intention, specifically $52.1 \%(p=.001)$; this finding is in alignment with previous TPB-based studies, where the constructs explained $14 \%$ to $55.1 \%$ of variation in intention. In addition, each construct contributed a significant, unique amount of variance in intention - attitude $9.7 \%(p=$ $.001)$, subjective norm $10.4 \%(p=.001)$, and perceived behavioral control $4.7 \%(p=.018)$; this, too, is consistent with previous TPB-based studies. Since all three constructs were significant predictors of intention, this outcome provides support for those areas in the CPAP-SAVER intervention in influencing CPAP adherence intention (and possibly, subsequent CPAP adherence behavior) and impetus for focus on those constructs in future testing of the CPAPSAVER intervention, especially attitude and subjective norm and the intervention components designed to impact these constructs.

\section{Prediction of CPAP Adherence Behavior}

The Theory of Planned Behavior suggests that attitude, subjective norm, and perceived behavioral control produce a relative behavioral intention and succeeding behavior (Ajzen, 2011). Ajzen (2011) proposes that intentions capture the motivating factors that influence eventual behavior. Behavior, then, can be predicted from intention, with the premise that the stronger the intention, the more likely the behavior.

In the CPAP-SAVER study, the logistic regression model was significant $(p=.002)$, where CPAP adherence intention explained between $14.1 \%$ and $21.0 \%$ of the variance in CPAP adherence behavior; prior TPB-based studies reported anywhere from $10.8 \%$ to $27.9 \%$. The CPAP-SAVER model also correctly classified a high number of cases (78.5\%). The odds ratio of 2.30 was significant $(p=.003)$, which indicated that participants with higher CPAP adherence intention were more than twice as likely to be CPAP adherent at one month; the model represented a level of $95 \%$ confidence that the actual value of the OR in the population was 
between 1.32 and 4.02. The model also reflected high sensitivity (true positives), positive predictive value, and negative predictive value, all of which support its strength and goodness of fit.

Facilitators and barriers. There are many facilitators and barriers to CPAP adherence behavior described in the literature, many of which were addressed in the CPAP-SAVER intervention study. Some of the facilitators previously reported include positive attitude to treatment, desire to avoid symptoms, fear of negative social consequences, feeling of physical improvement, and trust in healthcare professionals (Brostrom et al., 2010), as well as beliefs about OSA, presence of supportive relationships, and perceived physical and psychological benefits of using CPAP (Ayow et al., 2009). Barriers to CPAP adherence reported in the literature include negative attitude toward CPAP treatment, practical problems with and negative psychological effects of the CPAP equipment, side effects, and insufficient support from healthcare professionals and spouse (Brostrom et al., 2010). In a qualitative study by Sawyer et al. (2010), investigators found that CPAP adherers had fewer barriers to CPAP use and suggested that facilitators of CPAP use were less important later in treatment as users acclimated to use.

In previous CPAP adherence studies, positive attitude as a facilitator and negative attitude as a barrier were noted in several studies (Brostrom et al., 2010; Sawyer et al., 2010; Ward et al., 2014). Poulet et al. (2009) found anxiety to be a positive predictor of adherence; they also found that participants with maladaptive beliefs were significantly more likely to be nonadherent. Intervention participants in the CPAP-SAVER study had more positive attitudes toward CPAP adherence than the control (standard care) group at both one week and one month (based on the Theory of Planned Behavior attitude scores). More positive attitudes for the intervention group were not only noted in the attitude scores, but were also reflected in the 
Apnea Beliefs Scale scores at both one week and one month; intervention group participants had higher scores than the standard care group at both times $(p=.057)$. The more positive attitudes and beliefs manifested by the CPAP-SAVER intervention group may serve as motivators (intention) of their long-term adherence.

Feeling physical improvement from CPAP use (Brostrom et al., 2010) and perceived physical and psychological benefits (Ayow et al., 2009) have been reported as facilitators to CPAP adherence. Both daytime and nighttime symptoms, including excessive sleepiness, headaches, and disrupted sleep, have been reported as factors influencing CPAP use. In the CPAP-SAVER study, intervention participants reported excessive daytime sleepiness and gasping/choking at night as the most troublesome symptoms. The majority of the intervention participants reported improvement in these symptoms within the first few days of CPAP use, continuing into the next week of CPAP use.

Support from significant others and professionals was found to be a facilitator to CPAP adherence in previous work (Ayow et al., 2009, Brostrom et al., 2010; Ward et al., 2014). Conversely, insufficient support from healthcare professionals and spouse has been reported as a barrier to CPAP adherence (Brostrom et al., 2010). In a study by Baron et al. (2011), investigators found that perception of spousal involvement was significantly associated with higher three-month CPAP adherence. Hoy et al. (1999) found that intensive, nurse-led education and support offered greater improvements in symptoms, mood, and reaction time compared to standard care. During the support calls for the CPAP-SAVER study, the majority of participants reported that they had good support for their CPAP use from family/those closest to them. The majority also expressed confidence in the RT and home medical supplier to assist them with problems with their machine and/or mask if/when they arose. 
Practical problems with CPAP equipment have been reported as barriers to CPAP use (Brostrom et al., 2010), including mask difficulties, mask leakage, nasal/throat dryness, anxiety about the technology, and claustrophobic feelings. In a study of 350 patients wearing CPAP, the five most commonly reported issues were dry throat, uncomfortable pressure of the mask, feeling uncomfortable wearing CPAP in front of others, blocked up nose, and mask leaks (Brostrom et al., 2009). During support calls with intervention group participants in the CPAP-SAVER study, there were very few reported machine and/or mask problems. Of those problems reported, the most common were dryness of the nose/throat and mask leakage; these problems were easily resolved with humidity-setting changes and mask adjustments. No participants reported anxiety related to the machine or feeling uncomfortable wearing the mask in front of others; however, the question was posed as, "Are you having any machine or mask problems?" so they were not asked about specific issues.

Adherers and nonadherers. In a qualitative study by Sawyer et al. (2010), typologies of CPAP adherers and nonadherers were described (see Table 1).

Adherers perceived health and functional risks of untreated OSA, had positive belief in their ability to use CPAP from early in the diagnostic process, had clearly defined outcome expectations, had more facilitators than barriers as they progressed from diagnosis to treatment, and identified important social influences and support sources for pursuing both diagnosis and persisting with CPAP treatment. Nonadherers described not knowing the risks associated with OSA, perceived fewer symptoms of their diagnosis, did not have clearly defined outcome expectations for treatment, identified fewer improvements with CPAP exposure, placed less emphasis on social support and socially- 
derived feedback with early CPAP treatment, and perceived and experienced more barriers to CPAP treatment. (pp. $13-14$ )

They proposed that the differences between adherers and nonadherers center around knowledge, perception of self-efficacy, outcome expectancies and goals, and CPAP facilitators and barriers. This typology of CPAP adherers and nonadherers (see Table 1) and the proposed differences were considered in the development and testing of the CPAP-SAVER intervention. CPAP facilitators and barriers were addressed in the previous section of this dissertation, however, knowledge, perception of self-efficacy, and outcome expectancies and goals were also addressed in the intervention.

In previous research, intention to use CPAP was facilitated by education about OSA and its consequences (Tzischinsky et al., 2011). Increasing knowledge through education was the focus of the airway model, video, and education sheet components of the CPAP-SAVER intervention. The airway model served to educate intervention group participants about the OSA airway-brain mechanism and how CPAP works to splint the airway. The video provided education about the same topic and an additional visual aid to promote understanding of the OSA-CPAP airway effect. The education sheet highlighted the airway-brain mechanism of OSA, the risks associated with untreated OSA, and the benefits of CPAP (see Appendix C). Education about OSA and CPAP are essential in patients' understanding of OSA and its lifethreatening consequences. In addition, OSA knowledge has been shown to have an effect on CPAP attitude (Tyrell et al., 2006). Knowledge and attitude are both important constructs of the intention-behavior process (Ajzen, 2005).

Perception of self-efficacy has been shown to differentiate CPAP adherers and 
nonadherers (Sawyer et al., 2010). In the CPAP-SAVER study, self-efficacy was promoted with the use of a report card. The report card was designed to promote disease awareness and to serve as personalized performance feedback for the intervention group participants. The report card was used to document the participant's OSA severity (AHI), CPAP machine settings, CPAP machine use (mask-on time collected from the machine smart card), the participant's selfevaluation of his/her CPAP progress (self-rating of grade A [demonstrates adherence], B [showing steady progress], C [progressing, but with much support], or $\mathrm{N}$ [not adherent]), and the RT's evaluation of the participant's CPAP progress (same grading scale). The expected outcome was to increase participants' sense of self-efficacy and controllability. However, perceived behavioral control scores slightly decreased over time for both intervention and standard care group participants.

Outcomes expectancies and goals, another component of the typology differentiating adherers from nonadherers, focuses on costs and benefits influencing decisions to use CPAP or not (Sawyer et al., 2010). In the CPAP-SAVER study, the TPB questionnaire contained two questions that directly related to outcome expectations, I expect to adhere to my CPAP use every night as directed and I intend to adhere to my CPAP use every night as directed; most participants responded that they quite or extremely strongly agreed with these statements $(M=$ 6.65 and $M=6.72$ at baseline and $M=6.23$ and $M=6.22$ at one month). An independent samples t-test comparing the outcome expectancies-related questions for those participants meeting the definition of adherence and those not meeting the definition of adherence revealed a statistically significant difference at one week $(t[63]=-2.55, p=.013$ and $t[63]=-3.25, p=$ $.004)$ and one month $(t[63]=-2.55, p=.013$ and $t[63]=-3.09, p=.006)$, with adherers having higher means. In addition, intervention participants were asked about beliefs and intentions 
related to their CPAP adherence during the two support calls; most reported that they believed it was important to wear their CPAP every night.

Behavior change principles. Abraham and Michie (2008) developed a theory-linked taxonomy of behavior change techniques (BCT) to promote standardization and effectiveness of behavioral interventions. Seven of their techniques were applicable and operationalized in the CPAP-SAVER intervention study (see Table 2) with the expected outcome of promoting higher CPAP adherence in the intervention group. Technique 1, providing information about the behavior-health link, was defined by Abraham and Michie (2008) as providing general information about the behavioral risk; Technique 2, providing information on consequences, was defined as providing information about the benefits and costs of action or inaction. In the current study, both of these BCT were promoted through the use of the airway model, video, and education sheet; these intervention components were designed to improve participants' knowledge of OSA and its inherent risks, and the benefits of CPAP. Participants reported that they found the airway model somewhat or extremely helpful, and that the airway model and video motivated their CPAP use.

Four of Abraham and Michie's (2008) BCT were promoted through the use of the two support/subjective norm calls made to CPAP-SAVER intervention group participants; these BCT were numbers $3,4,6$, and 18 in the taxonomy. Technique 3 , providing information about others' approval, was defined as providing information about what others think as far as the person's behavior and whether others will approve or disapprove of the behavioral change (Abraham and Michie, 2008). Prompting intention formation, BCT 4, was defined as encouraging the person to act or set a general goal - to make a resolution to change. Taxonomy number 6 , provide general encouragement, was defined as praising or rewarding the participant for effort or performance. 
Technique number 18, using follow-up prompts, was defined as contacting the participant again after the main part of the intervention is complete (Abraham and Michie, 2008). In the CPAPSAVER study, the support/subjective normal calls made to intervention participants were designed to promote a subjective norm and these BCT. Participants were questioned and provided information about others' support and approval of their decision to treat their sleep apnea and their decision to wear CPAP. In addition, they were given praise about taking the initiative to get treatment for their sleep apnea and information about the importance of using their CPAP every night to cut down on health risks and improve their quality of life. The second support call, made after the main intervention components were completed, was intended to provide additional prompting as to subjective norm and follow-up encouragement to continue to adhere to their CPAP use. Participants reported a positive response to the support calls in that they liked them, understood them, and were motivated by them. The author noted that most participants expressed disappointment when she informed them that they would not be receiving further support calls after the second one.

Taxonomy BCT number 13, to provide feedback on performance, was defined by Abraham and Michie (2008) as providing data about recorded behavior or evaluating performance based on a set standard. The CPAP-SAVER intervention report card was designed to provide performance feedback to the participant to promote self-efficacy and controllability. The report card data included OSA-CPAP numbers and a grading system; this information was reviewed with intervention participants at baseline, during the first and second support calls, and upon completion of the study at one month.

\section{Strengths of the CPAP-SAVER Intervention Study}

The CPAP-SAVER intervention study was a theory-based, rigorous study with low 
attrition. The intervention was multidimensional, and focused on education, support, and behavior change. Experimental design was followed; participants were randomly assigned to groups, with a control group (standard care) used for comparison. Inclusion and exclusion criteria guided the recruitment and delimitation of the accessible population of participants. Intervention fidelity was maintained with the use of a protocol training manual and initial and booster training sessions to prepare the research assistants; monthly fidelity checks were conducted with no problems noted. Before data was collected, IRB approval and informed consent were obtained. Throughout the study, the Belmont Report Principles, including respect for persons, beneficence, and justice, were upheld. The intervention was well-received by participants, as evidenced by comments made during the support calls and the scores of 3 or 4 out of 4 on the Intervention Effectiveness Survey. The instruments used to collect data demonstrated prior and current internal consistency reliability within the sample.

Of the 24 intervention studies conducted in CPAP-naïve adults aged 18 and over from 2005 to present, 12 were theory based. Theory-based behavioral change interventions are thought to be more effective (Stepnowsky et al., 2013) and essential in fully understanding research-based evidence (Conn \& Groves, 2011). Of the 12 theory-based interventions reviewed, none were based on the Theory of Planned Behavior. The author believes that the current study is the first OSA-CPAP study using the Theory of Planned Behavior in its development and testing.

The Theory of Planned Behavior lent itself to successful use in the OSA-CPAP population, especially since the constructs (attitude, subjective norm, and perceived behavioral control) are important in facilitating behavioral intention (motivation) and actual behavior (CPAP adherence). Each component of the CPAP-SAVER intervention - support/subjective 
norm calls, airway model, video, education sheet, and report card - directly related to the theory and to recommended CPAP adherence facilitators (Sawyer et al., 2010) and behavior change techniques (Abraham \& Michie, 2008). With further testing, the CPAP-SAVER intervention has potential to become a standard-care protocol for OSA-CPAP management.

In the publications of the theory-based interventions reviewed for this dissertation, most of the authors did not address costs associated with their intervention, time or financial. Many of the studies involved potentially lengthy and costly therapy visits, such as motivational, social cognitive, or cognitive behavioral therapy; these visits may last hours, cost hundreds of dollars to actually implement in practice, and be burdensome to patients. The CPAP-SAVER intervention was both time- and cost-effective compared to other interventions. The intervention only added 22 minutes to the standard care time currently in place (12 minutes for the RT and 10 minutes for the nurse). To implement the CPAP-SAVER intervention, the only financial costs would be for the purchase of the airway model $(\$ 76.85)$ and paper copies (for the support call log, the education sheet, and the report card, a total of $\$ 0.55$ for each patient); the actual support calls and video had no associated costs. The intervention was nurse-led and involved respiratory therapists; these are individuals typically involved in standard-care education of OSA-CPAP patients. In addition, most of the CPAP-SAVER intervention was front-loaded into the first week of participants' CPAP use; since early CPAP use may be predictive of long-term use, this is important in promoting long-term CPAP adherence.

\section{Study Limitations}

There were a few limitations of the CPAP-SAVER intervention study, including sample size and diversity, study length, baseline knowledge level, and the Theory of Planned Behavior questionnaire format. The sample size was determined by power analysis to detect a difference 
in groups for the main research question (chi-square), however, the sample of 66 participants may have been too small for the ANOVA and regression analyses; following the $50+8 m$ guideline (Tabachnick \& Fidell, 2007), where $m$ equals the number of independent variables (predictors), a sample of at least 74 participants would be recommended to answer research question 8 regarding prediction of intention. This is further supported by the post hoc power analysis findings of 0.14 , which demonstrated that the study was underpowered. In addition, the sample was comprised of mainly white, middle-aged men; although OSA is most prevalent in the white male population, the results may not generalize to females or other ethnic groups. The convenience sample was mainly recruited from one home medical supply facility, with participants coming from areas in West Virginia, Maryland, and Pennsylvania; therefore, the results cannot be generalized. Testing the intervention in a larger, more diverse sample spanning a larger geographic area is needed.

The study length provided an additional limitation. Since this was a dissertation study, the study duration for each participant was limited to one month. However, during the first month of CPAP use, individuals were acclimating to the use of the machine, troubleshooting problems that arose, adapting to sleep while wearing a CPAP mask, and adjusting to life with CPAP as a new treatment. Most of the theory-based intervention studies reviewed for this dissertation spanned three months of participant involvement. Replicating the study in a larger, more diverse sample of participants over a longer period of time (at least three months), may provide better insight into the effect of the CPAP-SAVER intervention on long-term adherence, especially since insurance companies closely monitor patients' CPAP use over the first 90 days to determine adherence.

Baseline knowledge of OSA and CPAP may be another limitation. Even though the 
participants were CPAP-naïve and randomly assigned to groups, and the groups were determined to be homogeneous based on the demographics collected, participants may have differed in their baseline OSA-CPAP knowledge levels. Since CPAP adherence was high overall, regardless of group assignment, participants may have received varying degrees of education through their physician prior to their CPAP initiation or may have sought information through the internet or other resources upon diagnosis. An instrument to measure baseline OSA-CPAP knowledge, such as the Apnea Knowledge Test (Smith et al., 2004a), could be implemented with future testing of the CPAP-SAVER intervention to determine group homogeneity as to knowledge level.

The last limitation of the study related to the Theory of Planned Behavior Questionnaire. When the author typed the questions, they were spaced out over four pages to make them easier to read. However, participants had to turn back to page one many times while answering the questions to refer to the descriptors of the seven-point Likert scale. For ease in completion, the questionnaire could either be condensed into two pages or the Likert descriptors could be placed at the top of each page.

\section{Study Implications}

The CPAP-SAVER intervention study findings represent opportunities and implications for practice, nursing science, policy, and future research. These implications highlight the interactive nature of research, practice, and policy (Grady, 2015). This study also highlights the complex nature of CPAP adherence.

\section{Practice Implications}

Having an established OSA-CPAP standard to guide practitioners in working with newly diagnosed OSA patients acclimating to CPAP use may promote improved patient outcomes. 
During the author's observations following patients through the OSA diagnostic period, from office visit to sleep study to home medical supply facility, very little to no education about OSA was provided to patients; the most education provided was by the respiratory therapists in the sleep lab and the home medical supply facility, and related to the CPAP machine and its use. Since early and consistent education, support, and behavior-change motivation are important in promoting long-term CPAP adherence, these components should be included in the guidelines. These components may influence CPAP adherence beliefs, attitude, subjective norm, and perceived behavioral control to potentially promote a strong CPAP intention (motivation) and subsequent CPAP adherence.

Another practice implication relates to the CPAP user. In addition to education about OSA and the airway-brain mechanism, early and consistent support from healthcare providers and significant others is important. Through telephone calls and face-to-face meetings, nurses may promote a more positive CPAP attitude and stronger subjective norm and sense of selfefficacy to empower the CPAP user, influence CPAP motivation, and promote long-term adherence. Emphasizing risks associated with OSA, benefits of CPAP, facilitators and barriers to CPAP use, behavioral change techniques, and OSA-CPAP problem solving, nurses may have significant impact on patient outcomes of CPAP adherence. Educating significant others about the influence they may have on their loved one's CPAP adherence and involving them in each step throughout the OSA-CPAP trajectory are imperative.

\section{Nursing Science and Policy Implications}

The nursing profession has the potential to be at the forefront in making positive changes in healthcare quality, delivery, and efficiency that have tremendous impact on the lives of patient, families, and communities (Weston, White, \& Peterson, 2013). As part of its key 
messages, the Institute of Medicine Report (2011) calls on nurses to be full partners in redesigning the healthcare system and engage in more effective workforce planning and policymaking with better data collection. Nurses, and especially nurse scientists, must be more involved in conducting high-impact research studies and translating the findings of those studies into policy. It is imperative that nurse scientists develop "political acumen" (Shamian \& Griffin, 2003, p. 49) and endorse their research to influence decision making in healthcare policy (O’Brien-Pallas, 2003).

One of the most consistent findings from clinical and health services research is the failure to translate research into practice and policy. As a result of these evidencepractice and policy gaps, patients fail to benefit optimally from advances in healthcare and are exposed to unnecessary risks of iatrogenic harms, and healthcare systems are exposed to unnecessary expenditure resulting in significant opportunity costs. (Grimshaw et al., 2012, p. 1)

As nurse scientists, we must strengthen our voice and advocate for the value of our research and other contributions in making significant changes, even paradigm shifts, in the healthcare system. In addition, more nursing involvement on committees and task forces impacting healthcare and research decision making is paramount in making nursing's presence known.

This phenomenon is evident in the field of sleep science. There are two guidelines to aid practitioners in diagnosing OSA, determining treatment options, and establishing the need for CPAP, one developed by the American Academy of Sleep Medicine (Epstein et al., 2009) and one developed by the American College of Physicians (Qaseem et al., 2013). However, there is no detailed protocol to provide specific guidance to practitioners in OSA-CPAP patient education, support, and behavior change in initiating and sustaining CPAP adherence. The 
clinical guideline established by the American Academy of Sleep Medicine (Epstein et al., 2009) is becoming an outdated document. In the guideline (Epstein et al., 2009), education components to be covered by the sleep specialist are suggested, however, patients may not see a sleep specialist in the OSA-CPAP trajectory; the sleep study may be ordered by the primary care provider as the gatekeeper of the patient's care. In addition, the sleep specialist may tend to focus more on making the OSA diagnosis and not on OSA education since the diagnosis is not established until a sleep study is conducted and the results are compiled. Thus, the patient may move through the unfamiliar OSA diagnosis trajectory with little guidance and understanding. Nurse scientists have the power to affect change in this area; however, nurses are not represented on either committee. Increasing nursing's presence in adherence-related research and policy are critical.

Grady (2015) suggests that, ideally, policy is formulated by evidence that informs best solutions or strategies and emphasizes implementation science in the translation process (Grady, 2011). The CPAP-SAVER study, in the context of previous work and the need for an updated, detailed guideline, provides the impetus and groundwork for the establishment of a current, comprehensive protocol that can be implemented by nurses. However, single studies rarely provide sufficient evidence for policy change (Grimshaw et al., 2012); replication of the CPAPSAVER intervention in a larger, more diverse sample and synthesizing the results with other intervention studies are necessary to begin the process of this research-to-policy translation. According to Grady (2011, p. 17), "With the coming of age of nursing research, we have a new opportunity and a new imperative to generate and implement the results of well-designed studies to provide the foundation for evidence-based practice and policies." Nursing research that shapes policy is considered to have the highest impact in healthcare delivery, especially research 
that contributes to shifts in paradigm, how clinical problems are viewed, identification of new research priorities, or the establishment of new regulations or patents (Austin, 2016). The findings from the CPAP-SAVER study add to the body of knowledge of CPAP adherence and provide underpinnings for the development of a more comprehensive OSA-CPAP protocol that can be implemented by nurses, especially since nurses have extensive training in and experience with patient education and support.

Findings from the CPAP-SAVER study have potential to contribute to policy formation in the area of CPAP adherence, especially related to positive attitude formation, subjective norm promotion, and perceived behavioral control enhancement. However, the research evidence is just a start; to facilitate the process of research-to-policy translation, nurse scientists must have knowledge of the policymaking process and exercise their power in influencing change (Grady, 2011). Important first steps in promoting policy development include identifying needs or gaps, benefits and benefactors of the change, support networks, and stakeholders and innovators (Grady, 2011). Using established frameworks and models (Shamian \& Shamian-Ellen, 2011), including a health policy toolkit (Kostas-Polston et al., 2015), to systematically guide the research-to-policy-translation process and networking with key stakeholders who have strong influence in policymaking decisions may assist nurse scientists to more effectively and efficiently move issues through the research-to-policy trajectory (Hinshaw \& Grady, 2011). Increased nurse scientist involvement in nursing, research, and government organizations may facilitate this process. These are next steps in the process of translating the research findings from the CPAP-SAVER study to eventual policy.

Nurse scientists need to charter new directions and develop new ways of thinking to influence practice and inform health policy (Hinshaw \& Grady, 2011); this is true of nurse 
scientists in the field of sleep science as well, since OSA-CPAP research is a timely issue that has economic impact and strong potential to influence patient outcomes related to morbidity, mortality, and quality of life. More nursing involvement in sleep organizations and serving as primary investigators for OSA-CPAP research studies to advance nursing's presence in the field of sleep science are needed. Presenting research findings to sleep organizations, as well as increasing membership and committee involvement, may aid nurse scientists in promoting consensus and support in the eventual establishment of a protocol for improving OSA management and sustaining CPAP adherence. Dissemination of work, including the findings of intervention studies such as the CPAP-SAVER study, and clear communication of the findings of such studies to various stakeholders augment the importance of nursing science and its powerful influence on health policy.

\section{Research Implications: Recommendations for Future Research}

More current research is needed in the area of CPAP adherence, especially intervention studies and implementation science. Many of the classic works in the field of OSA-CPAP research are dated; people and their patterns of behavior change over time as trends change and generations pass. The CPAP-SAVER study builds upon and enhances seminal work previously

conducted in the field, with new ideas for future research. Replicating the CPAP-SAVER study in a larger, more diverse sample is a first step in translating its findings to practice and policy. The author poses recommendations for future research related to the lessons learned from the CPAP-SAVER study, as well as other recommendations in the field of sleep science. These recommendations include further research examining the subjective norm construct, further testing of the report card, future research testing the Theory of Planned Behavior in other OSACPAP populations, and conducting future research using CPAP adherence as the dependent 
variable (as opposed to CPAP use). These recommendations represent strategies and opportunities for advancing nursing scientists' status in the field of sleep science.

Subjective norm was the strongest, unique predictor of CPAP intention in the CPAPSAVER sample. Based on this finding and previous works, further examination of the amount and types of pressure/support provided to CPAP users is recommended. Accepting and acclimating to CPAP use can be very difficult for new users; extensive support early in the diagnosis is important in promoting long-term adherence. Using the framework of the CPAPSAVER study, examining the dosage strength, timing, and frequency of the support calls warrants further study. Promoting a stronger sense of subjective norm, through increased pressure by healthcare providers and significant others, may improve CPAP adherence. Improved support mechanisms may successfully guide the CPAP user through the first few months of use on the path to long-term adherence.

Further testing of the use of report cards in the OSA-CPAP population is recommended. Again, dosage strength, timing, and frequency may have played a part in the lack of significance in this area of the CPAP-SAVER study, however, report card use may have promise in promoting a stronger sense of self-efficacy and control that may influence CPAP intention and actual adherence behavior. Examining the effect of the use of phone applications on perceived behavioral control (self-efficacy) and subsequent CPAP adherence behavior is also recommended.

More OSA-CPAP studies examining the use of the Theory of Planned Behavior as a framework for intervention research and implementation science is recommended. In the CPAPSAVER study, all three major constructs - attitude, subjective norm, and perceived behavioral control - were significant, unique predictors of CPAP intention; in turn, CPAP adherence 
intention significantly predicted CPAP adherence behavior. Testing the theory in larger, more diverse populations may clarify the utility of the theory and its constructs in translating research findings into healthcare policy, especially in the development of a detailed protocol for OSACPAP management.

Lastly, the author recommends further theory-based intervention research using CPAP adherence as the dependent variable. Many published studies indicate that the research is in the area of CPAP adherence, however, in reading the publication, it is noted that CPAP use is measured. In order for CPAP machines to be covered by insurance companies, patients must meet the standard of being CPAP adherent. Many insurance companies follow the guidelines of the Centers for Medicare and Medicaid Services (2013), requiring CPAP use for four hours per night for $70 \%$ of the nights over 30 consecutive days in the first three-month period, for the patient to be considered CPAP adherent. Many patients would not be able to afford to buy the CPAP machine outright, so meeting this adherence guideline is critical in treating OSA. The CPAP-SAVER study used this more stringent variable; more research is needed following this lead.

\section{Conclusions}

Much was learned from this dissertation study testing the theory-based, multidimensional CPAP-SAVER intervention. Chi-square testing revealed no statistically significant effect of the intervention on one-month CPAP adherence. In comparison to standard care, the intervention group used CPAP more total hours at one week, more hours per night at one week, and more nights over one week and one month. Mixed between-within ANOVA testing demonstrated a significant effect of time on anxiety as a background factor and CPAP adherence intention, however there was no significant effect of the intervention on adherence attitude, subjective 
norm, or perceived behavioral control. The between-subjects effect of the intervention on adherence beliefs approached significance $(p=.057)$ and was supported by t-test results, with intervention participants demonstrating higher mean one-month CPAP adherence beliefs than the standard care group. Significant differences in CPAP adherence attitude, subjective norm, and perceived behavioral control scores over time were not noted between the groups. However, CPAP adherence attitude, subjective norm, and perceived behavioral control were significant predictors of CPAP adherence intention and explained $52.1 \%$ of the total variance in CPAP adherence intention; each of the three variables demonstrated a significant, unique contribution to the variance in CPAP adherence intention scores. In addition, CPAP adherence intention significantly explained between $14.1 \%$ and $21.0 \%$ of the variance in CPAP adherence behavior, with high sensitivity and positive predictive value. Most intervention group participants rated the CPAP-SAVER intervention components as 3 or 4 (somewhat or extremely helpful, liked, understood, and motivating) on a Likert scale of 0 to 4 . Further testing of the intervention is needed, especially in a larger, more diverse sample, to determine its full impact on CPAP adherence. With further testing and development, the CPAP-SAVER intervention may have implications for protocol and policy development to become the standard of care in OSA-CPAP management.

\section{Summary}

This chapter presented a discussion of the findings of the CPAP-SAVER intervention study and the theoretical constructs upon which the intervention and study were based. CPAP facilitators and barriers, the typologies of adherers (Sawyer et al., 2010), and the behavior change taxonomy (Abraham \& Michie, 2008) were discussed in the context of the current study. A discussion of the findings in the context of the study components and previous works was 
presented. Implications of the CPAP-SAVER intervention study findings for practice, nursing science, policy, and future research were summarized. 


\section{References}

Aboussouan, L., Zahand, Z., \& Podmore, P. (2010). This thing is impossible! In N. FoldvarySchaefer, J. Krishna, \& K. Budur (Eds.), A case a week: Sleep disorders from the Cleveland clinic (pp. 151-159). New York, NY: Oxford University Press.

Abraham, C., \& Michie, S. (2008). A taxonomy of behavior change techniques used in interventions. Health Psychology, 27, 379-387. doi: 10.1037/0278-6133.27.3.379

Ahmad, M. H., Shahar, S., Mohd, N. I., Teng, F., Manaf, Z. A., Sakian, N. I. M., \& Omar, B. (2014). Applying Theory of Planned Behavior to predict exercise maintenance in sarcopenic elderly. Clinical Interventions in Aging, 9, 1551-1561. doi: 10.2147/ CIA.S60462

Ajzen, I. (1985). From intentions to action: A theory of planned behavior. In J. Kuhl \& J. Beckman (Eds.), Action-control: From cognition to behavior (pp. 11-39). Heidelberg, Germany: Springer.

Ajzen, I. (1991). The theory of planned behavior. Organizational Behavior and Human Decision Processes, 50, 179-211. doi: 10.1016/0749-5978(91)90020-T

Ajzen, I. (2005). Attitudes, personality and behavior ( $2^{\text {nd }}$ ed.). Maidenhead, UK: Open University Press.

Ajzen, I. (2006). Behavior. Retrieved from http://people.umass.edu/aizen/beh.html Ajzen, I. (2011). Behavioral interventions: Design and evaluation guided by the Theory of Planned Behavior. In M. M. Mark, S. I. Donaldson, \& B. Campbell (Eds.), Social psychology and evaluation (pp. 74-100). New York, NY: Guilford Press.

Akashiba, T., Kawahara, S., Akahoshi, T., Omori, C., Saito, O., Majima, T., \& Horie, T. (2002). Relationship between quality of life and mood or depression in patients with severe 
obstructive sleep apnea syndrome. Chest, 122, 861-865.

Aloia, M. S. (2011). Understanding the problem of poor CPAP adherence. Sleep Medicine Reviews, 15, 341-342. doi: 10.1016/j.smrv.2011.04.002

Aloia, M. S., Arnedt, J. T., Riggs, R. L., Hecht, J., \& Borrelli, B. (2004). Clinical management of poor adherence to CPAP: Motivational enhancement. Behavioral Sleep Medicine, 2, 205222. doi: 10.1207/s15402010bsm0204_3

Aloia, M. S., Arnedt, J. T., Stanchina, M., \& Millman, R. P. (2007). How early in treatment is PAP adherence established? Revisiting night-to-night variability. Behavioral Sleep Medicine, 5, 229-240. doi: 10.1080/15402000701264005

Aloia, M. S., Arnedt, J. T., Stepnowsky, C., Hecht, J., \& Borrelli, B. (2005). Predicting treatment adherence in obstructive sleep apnea using principles of behavior change. Journal of Clinical Sleep Medicine, 1, 346-353.

Aloia, M. S., Smith, K., Arnedt, J. T., Millman, R. P., Stanchina, M., Carlisle, C., Hecht, J., \& Borrelli, B. (2007). Brief behavioral therapies reduce early positive airway pressure discontinuation rates in sleep apnea syndrome: Preliminary findings. Behavioral Sleep Medicine, 5, 89-104. doi: 10.1080/15402000701190549

American Academy of Sleep Medicine. (2008). Obstructive sleep apnea. Darien, IL: Author. American Academy of Sleep Medicine. (2014). Rising prevalence of sleep apnea in US threatens public health. Retrieved from http://www.aasmnet.org/articles.aspx?id=5043

Andrews, J. G., \& Oei, T. P. S. (2004). The roles of depression and anxiety in the understanding and treatment of obstructive sleep apnea syndrome. Clinical Psychology Review, 24, 1031-1049. doi: 10.1016/j.cpr.2004.08.002

Antic, N. A., Catcheside, P., Buchan, C., Hensley, M., Naughton, M. T., Rowland, S., . . . 
McEvoy, R. D. (2011). The effect of CPAP in normalizing daytime sleepiness, quality of life, and neurocognitive function in patients with moderate to severe OSA. Sleep, 34, 111-119.

Armitage, C. J. (2005). Can the Theory of Planned Behavior predict the maintenance of physical activity? Health Psychology, 24, 235-245. doi: 10.1037/0278-6133.24.3.235

Austin, J. K. (2016). NINR midcareer technical workshop 2b: Align your program of research with criteria for promotion. Retrieved from https://www.youtube.com/watch?v=nf3QRwRVfU

Ayow, T. M., Paquet, F., Dallaire, J., Purden, M., \& Champagne, K. A. (2009). Factors influencing the use and nonuse of continuous positive airway pressure therapy: A comparative case study. Rehabilitation Nursing, 34, 230-236.

Bandura, A. (2004). Health promotion by social cognitive means. Health Education and Behavior, 31, 143-164. doi: 10.1177/1090198104263660

Baron, K. G., Berg, C. A., Czajowski, L. A., Smith, T. W., Gunn, H. E., Jones, C. R. (2011). Sleep Breath, 15, 599-606. doi: 10.1007/s11325-010-0409-5

Bartlett, D., Wong, K., Richards, D., Moy, E., Espie, C. A., Cistulli, P. A., \& Grunstein, R. (2013). Increasing adherence to obstructive sleep apnea treatment with a group Social Cognitive Therapy treatment intervention: A randomized trial. Sleep, 30, 1647-1654.

Basoglu, O. K., Midilli, M., Midilli, R., \& Bilgen, C. (2012). Adherence to continuous positive airway pressure therapy in obstructive sleep apnea syndrome: Effect of visual education. Sleep Breath, 16, 1193-1200. doi: 10.1007/s11325-011-0631-9

Beck, A. T., Epstein, N., Brown, G., \& Steer, R. A. (1988). An inventory for measuring clinical anxiety: Psychometric properties. Journal of Consulting and Clinical Psychology, 56, 
893-897. doi: 10.1037/0022-006x.56.6.893

Beck, A. T., \& Steer, R. A. (1993). Beck Anxiety Inventory (1993 ed.). San Antonio, TX: The Psychological Corporation.

Benton, D. (2012). Advocating globally to shape policy and strengthen nursing's influence. Online Journal of Issues in Nursing, 17(1), 1-11.

Blackwell, P. (2005). The Theory of Planned Behavior and treatment adherence in asthma patients (Doctoral dissertation). Retrieved from ProQuest (UMI No. 3179455).

Booth, A. R., Norman, P., Goyder, E., Harris, P. R., \& Campbell, M. J. (2014). Pilot study of a brief intervention based on the theory of planned behaviour and self-identity to increase chlamydia testing among young people living in deprived areas. British Journal of Health Psychology, 19, 636-651. doi: 10.1111/bjhp.12065

Brostrom, A., Johansson, P., Stromberg, A., Albers, J., Martensson, J., \& Svanborg, E. (2007). Obstructive sleep apnoea syndrome: Patients' perceptions of their sleep and its effects on their life situation. Journal of Advanced Nursing, 57, 318-327. doi: 10.1111/j.1365-2648. 2006.04110.x

Brostrom, A., Nilsen, P., Johansson, P., Ulander, M., Stromberg, A., Svanborg, E., \& Fridlund, B. (2010). Putative facilitators and barriers for adherence to CPAP treatment in patients with obstructive sleep apnea syndrome: A qualitative content analysis. Sleep Medicine, 11, 126-130. doi: 10.1016/j.sleep.2009.04.010

Brostrom, A., Stromberg, A., Ulander, M., Fridlund, B., Martensson, \& Svanborg, E. (2009). Perceived informational needs, side-effects and their consequences on adherence- $\mathrm{A}$ comparison between CPAP treated patients with OSAS and healthcare personnel. Patient Education and Counseling, 74, 228-235. doi: 10.1016/j.pec.2008.08.012

Brostrom, A., Ulander, M., Nilsen, P., Svanborg, E., \& Arestedt, K. F. (2011). The attitudes to 
CPAP treatment inventory: Development and initial validation of a new tool for measuring attitudes to CPAP treatment. Journal of Sleep Research, 20, 460-471. doi: $10.1111 / \mathrm{j} .1365-2869.2010 .00885 . \mathrm{x}$

Budhiraja, R., Parthasarathy, S., Drake, C. L., Roth, T., Sharief, I., Budhiraja, P., ... Hudgel, D. W. (2007). Early CPAP use identifies subsequent adherence to CPAP therapy. Sleep, 30, 320-324.

Canessa, N., Castronovo, V., Cappa, S. F., Aloia, M. S., Marelli, S., Falini, A., ... FeriniStrambi, L. (2011). Obstructive sleep apnea: Brain structural changes and neurocognitive function before and after treatment. American Journal of Respiratory \& Critical Care Medicine, 183, 1419-1426. doi: 10.1164/rccm.201005-06930C

Centers for Medicare and Medicaid Services. (2013). Continuous and bi-level positive airway pressure (CPAP/BPAP) devices: Complying with documentation \& coverage requirements. Retrieved from http://www.cms.gov/Outreach-and-Education/MedicareLearning-Network-MLN/MLNProducts/downloads/PAP_DocCvg_Factsheet_ ICN905064.pdf

Cohen, J. (1988). The analysis of variance. In Statistical power analysis for the behavioral sciences ( $2^{\text {nd }}$ ed., pp. 284-288). Retrieved from http://www.lrdc.pitt.edu/schneider/ p2465/Readings/Cohen,\%201988\%20(Statistical\%20Power,\%20273-406).pdf

Cohen, J. (1992). A power primer. Psychological Bulletin, 112, 155-159.

Conn, V. S. (2012). Unpacking the black box: Countering the problem of inadequate intervention descriptions in research reports. Western Journal of Nursing Research, 34, 427-433. doi: 10.1177/0193945911434627

Conn, V. S., \& Groves, P. S. (2011). Protecting the power of interventions through proper 
reporting. Nursing Outlook, 59, 318-325. doi: 10.1016/j.outlook.2011.06.003

DeCoster, J., \& Iselin, A.-M. (2006). Double Data Entry. [Computer Software]. Charlottesville, VA: Author.

Deng, T., Wang, Y., Sun, M., \& Chen, B. (2013). Stage-matched intervention for adherence to CPAP in patients with obstructive sleep apnea: A randomized controlled trial. Sleep Breath, 17, 791-801. doi: 10.1007/s11325-012-0766-3

Dickerson, S. S., \& Akhu-Zaheya, L. (2007). Life changes in individuals diagnosed with sleep apnea while accommodating to continuous positive airway pressure (CPAP) devices. Rehabilitation Nursing, 32, 241-250.

Epstein, L. J., Kristo, D., Strollo, P. J., Jr., Friedman, N., Malhotra, A., Patil, S. P., . . . Weinstein, M. D. (2009). Clinical guideline for the evaluation, management and longterm care of obstructive sleep apnea in adults. Journal of Clinical Sleep Medicine, 5, 263276.

Falcone, V. A., Damiani, M. F., Quaranta, V. N., Capozzolo, A., \& Resta, O. (2014). Polysomnograph chart view by patients: A new educational strategy to improve CPAP adherence in sleep apnea therapy. Respiratory Care, 59, 193-198. doi: 10.4187/resp care. 02491

Faul, F., Erdfelder, E., Lang, A. G., \& Buchner, A. (2007). G*Power 3: A flexible statistical power analysis program for the social, behavioral, and biomedical sciences. Behavior Research Methods, 39, 175-191.

Fishbein, M., \& Ajzen, I. (1975). Belief, attitude, intention, and behavior: An introduction to theory and research. Reading, MA: Addison-Wesley.

Francis, J., Eccles, M. P., Johnston, M., Walker, A. E., Grimshaw, J. M., Foy, R., ... Bonetti, D. 
(2004). Constructing questionnaires based on the theory of planned behaviour: A manual for health services researchers. Newcastle upon Tyne,

UK: Centre for Health Services Research, University of Newcastle upon Tyne.

Gay, P., Weaver, T., Loube, D., \& Iber, C. (2006). Evaluation of positive airway pressure treatment for sleep related breathing disorders in adults. Sleep, 29, 381-401.

Glebocka, A., Kossowska, A., \& Bednarek, M. (2006). Obstructive sleep apnea and the quality of life. Journal of Physiology and Pharmacology, 57, 111-117.

Grady, P. (2011). Research: A foundation for health policy. In A. S. Hinshaw \& P. A. Grady (Eds.), Shaping health policy through nursing research (pp. 17-33). New York, NY: Springer Publishing Company.

Grady, P. (2015). Translating nursing research into policy. AACN Student Policy Summit: March 23, 2015. Retrieved from http://www.aacn.nche.edu/government-affairs/Grady.pdf

Grimshaw, J. M., Eccles, M. P., Lavis, J. N., Hill, S. J., \& Squires, J. E. (2012). Knowledge translation of research findings. Implementation Science, 7(50), 1-17.

Hinshaw, A. S., \& Grady, P. A. (2011). Forging the missing link: From nursing research to health policy. In A. S. Hinshaw \& P. A. Grady (Eds.), Shaping health policy through nursing research (pp. 281-292). New York, NY: Springer Publishing Company.

Hoy, C. J., Vennelle, M., Kingshott, R. N., Engleman, H. M., \& Douglas, N. J. (1999). Can intensive support improve continuous positive airway pressure use in patients with the sleep apnea/hypopnea syndrome? American Journal of Respiratory Critical Care Medicine, 159, 1096-1100.

IBM. (2016). IBM SPSS Statistics Version 24. [Computer Software]. Armonk, NY: Author. Institute of Medicine. (2011). Future of nursing: Leading change, advancing health. 
Washington, DC: The National Academies Press.

Kagee, A., \& Merwe, M. (2006). Predicting treatment adherence among patients attending primary health care clinics: The utility of the Theory of Planned Behaviour. South African Journal of Psychology, 36, 699-714.

Kapur, V. K. (2010). Obstructive sleep apnea: Diagnosis, epidemiology, and economics. Respiratory Care, 55, 1155-1167.

Karalis, M. (2001). Hemodialysis and peritoneal dialysis report cards. Journal of Renal Nutrition, 11, 49-53. doi: 10.1053/jren.2001.20093

Kjelsberg, F. N., Ruud, E. A., \& Stavem, K. (2005). Predictors of symptoms of anxiety and depression in obstructive sleep apnea. Sleep Medicine, 6, 341-346.

Kostas-Polston, E. A., Thanavaro, J., Arvidson, C., \& Morritt Taub, L.-F. (2015). Advanced practice nursing: Shaping health through policy. Journal of the American Association of Nurse Practitioners, 27, 11-20.

Kothe, E. J., Mullan, B. A., \& Butow, P. (2012). Promoting fruit and vegetable intake: Testing an intervention based on the Theory of Planned Behavior. Appetite, 58, 997-1004. doi: 10.1016/j.appet.2012.02.012

Kreivi, H. -R, Maasilta, P., \& Bachour, A. (2014). Willingness score obtained after a short trial predicts CPAP use at 1 year. Sleep Breath, 18, 207-213. doi: 10.1007/s11325-013-0872-x

Kumar, R., Macey, P. M., Cross, R. L., Woo, M. A., Yan-Go, F. L., \& Harper, R. M. (2009). Neural alterations associated with anxiety symptoms in obstructive sleep apnea syndrome. Depression and Anxiety, 26, 480-491. doi: 10.1002/da.20531

Lai, A. Y. K., Fong, D. Y. T., Lam, J. C. M., Weaver, T. E., \& Ip, M. S. M. (2014). The efficacy of a brief motivational enhancement education program on CPAP adherence in 
OSA: A randomized controlled trial. Chest, 146, 600-610. doi: 10.1378/chest.13-2228

Lewis, K. E., Bartle, I. E., Watkins, A. J., Seale, L., \& Ebden, P. (2006). Simple interventions improve re-attendance when treating the sleep apnoea syndrome. Sleep Medicine, 7, 241247. doi: 10.1016/j.sleep.2005.09.007

Lewis, K. E., Seale, L., Bartle, I. E., Watkins, A. J., \& Ebden, P. (2004). Early predictors of CPAP use for the treatment of obstructive sleep apnea. Sleep, 27, 134-138.

Loube, D. I., \& Andrada, T. F. (1999). Comparison of respiratory polysomnographic parameters in matched cohorts of upper airway resistance and obstructive sleep apnea syndrome patients. Chest, 115, 1519-1524. doi: 10.1378/chest.115.6.1519

McClean, K. M., Kee, F., Young, I. S., \& Elborn, J. S. (2008). Obesity and the lung: 1 • Epidemiology. Thorax, 63, 649-654. doi: 10.1136/thx.2007.086801

McConnon, A., Raats, M., Astrup, A., Bajzova, M., Handjieva-Darlenska, T., Lindroos, A. K., ... Shepherd, R. (2012). Application of the Theory of Planned Behavior to weight control in an overweight cohort: Results from a pan-European dietary intervention trial (DiOGenes). Appetite, 58, 313-318. doi: 10.1016/j.appet.2011.10.017

Meurice, J. C., Ingrand, P., Portier, F., Rakotonanahari, D., Fournier, E., Philip-Joet, F., \& Veale, D. (2007). A multicentre trial of education strategies at CPAP induction in the treatment of severe sleep apnoea-hypopnoea syndrome. Sleep Medicine, 8, 37-42. doi: 10.1016/j. sleep.2006.05.010

Milton, A. C., \& Mullan, B. A. (2012). An application of the Theory of Planned Behavior-A randomized controlled food safety pilot intervention for young adults. Health Psychology, 31, 250-259. doi: 10.1037/a0025852

Montanaro, E. A., \& Bryan, A. D. (2014). Comparing theory-based condom interventions: 
Health Belief Model versus Theory of Planned Behavior. Health Psychology, 33, 12511260. doi: $10.1037 / \mathrm{a} 0033969$

National Commission for the Protection of Human Subjects of Biomedical and Behavioral Research. (1979). The Belmont Report: Ethical principles and guidelines for the protection of human subjects of research. Washington, DC: Department of Health, Education, and Welfare. Retrieved from http://www.hhs.gov/ohrp/humansubjects/ guidance/belmont.html

Newman, M. A., Smith, M. C., Pharris, M. D., \& Jones, D. (2008). The focus of the discipline revisited. Advances in Nursing Science, 31(1), E16-E27.

Niven, A., Nevill, A., Sayers, F., \& Cullen, M. (2012). Predictors of rehabilitation intention and behavior following anterior cruciate ligament surgery: An application of the Theory of Planned Behavior. Scandinavian Journal of Medicine \& Science in Sports, 22, 316-322. doi: 10.1111/j.1600-0838.2010.01236.x

O’Brien-Pallas, L. (2003). Leadership in research: About building relationships. Canadian Journal of Nursing Leadership, 16, 28-31.

Olsen, S., Smith, S., Oei, T., \& Douglas, J. (2008). Health belief model predicts adherence to CPAP before experience with CPAP. European Respiratory Journal, 32, 710-717. doi: $10.1183 / 09031936.00127507$

Olsen, S., Smith, S., \& Oei, T. P. S. (2008). Adherence to continuous positive airway pressure therapy in obstructive sleep apnoea sufferers: A theoretical approach to treatment adherence and intervention. Clinical Psychology Review, 28, 1355-1371. doi: 10.1016/j.cpr.2008.07. 004

Olsen, S., Smith, S., Oei, T. P. S., \& Douglas, J. (2012). Motivational interviewing (MINT) 
improves continuous positive airway pressure (CPAP) acceptance and adherence: A randomized controlled trial. Journal of Consulting and Clinical Psychology, 80, 151-163. doi: $10.1037 / \mathrm{a} 0026302$

O’Neill, S. C., Bowling, J. M., Brewer, N. T., Lipkus, I. M., Skinner, C. S., Strigo, T. S., \& Rimer, B. K. (2008). Intentions to maintain adherence to mammography. Journal of Women's Health, 17, 1133-1141. doi: 10.1089/jwh.2007.0600

Pack, A. I. (2006). Advances in sleep-disordered breathing. American Journal of Respiratory \& Critical Care Medicine, 173, 7-15. doi: 10.1164/rccm.200509-1478OE

Pallant, J. (2016). SPSS survival manual: A step by step guide to data analysis using IBM SPSS ( $6^{\text {th }}$ ed.). Berkshire, England: McGraw Hill Education.

Park, J. G., Ramar, K., \& Olson, E. J. (2011). Updates on definition, consequences, and management of obstructive sleep apnea. Mayo Clinic Proceedings, 86, 549-555. doi: 10.4065/mcp. 2010.0810

Parthasarathy, S., Wendel, C., Haynes, P. L., Atwood, C., \& Kuna, S. (2013). A pilot study of CPAP adherence promotion by peer buddies with sleep apnea. Journal of Clinical Sleep Medicine, 9, 543-550. doi: 10.5664/jcsm.2744

Pawlina, W., \& Drake, R. L. (2013). Anatomical models: Don't banish them from the anatomy laboratory yet. Anatomical Sciences Education, 6, 209-210. doi: 10.1002/ase.1380

Peppard, P. E., Young, T., Barnet, J. H., Palta, M., Hagen, E. W., \& Hia, K. M. (2013). Increased prevalence of sleep-disordered breathing in adults. American Journal of Epidemiology, 177, 1006-1014. doi: 10.1093/aje/kws342

Physician group builds tools to help practitioners boost diabetes outcomes. (2003, April). Disease Management Advisor, 9, 54-55. 
Polit, D. F., \& Beck, C. T. (2012). Nursing research: Generating and assessing evidence for nursing practice (9th ed.). Philadelphia, PA: Walters Kluwer Health/Lippincott Williams \& Wilkins.

Poulet, C., Veale, D., Arnol, N., Levy, P., Pepin, J. L., \& Tyrrell, J. (2009). Psychological variables as predictors of adherence to treatment by continuous positive airway pressure. Sleep Medicine, 10, 993-999. doi: 10.1016/j.sleep.2009.01.007

Punjabi, N. M. (2008). The epidemiology of adult obstructive sleep apnea. Proceedings of the American Thoracic Society, 5, 136-143. doi: 10.1513/pats.200709-155MG

Qaseem, A., Holty, J. E. C., Owens, D. K., Dallas, P., Starkey, M., \& Shekelle, P. (2013). Management of obstructive sleep apnea in adults: A clinical practice guideline from the American College of Physicians. Annals of Internal Medicine, 159, 471-483.

Rakel, D. P., \& Jonas, W. B. (2016). Empowerment. In R. E. Rakel and D. P. Rakel Textbook of family medicine ( $9^{\text {th }}$ ed., pp. 20-21). Philadelphia, PA: Elsevier.

Rakel, R. E. (2009). Clinical and societal consequences of obstructive sleep apnea and excessive daytime sleepiness. Postgraduate Medicine, 121, 86-95. doi: 10.3810/pgm.2009.01.1957

Reishtein, J., Pack, A. I., Maislin, G., Dinges, D. F., Bloxham, T. J., George, C. F. P., \& Greenberg, H. (2006). Sleepiness and relationships in obstructive sleep apnea. Issues in Mental Health Nursing, 27, 319-330.

Richards, D., Bartlett, D. J., Wong, K., Mauloff, J., \& Grunstein, R. R. (2007). Increased adherence to CPAP with a group cognitive behavioral treatment intervention: A randomized trial. Sleep, 30, 635-340.

Roecklein, K. A., Schumacher, J. A., Gabriele, J. M., Fagan, C., Baran, A. S., \& Richert, A. C. (2010). Personalized feedback to improve CPAP adherence in obstructive sleep apnea. 
Behavioral Sleep Medicine, 8, 105-112. doi: 10.1080/15402001003622859

Sabate, E. (2003). Adherence to long-term therapies: Evidence for action. Geneva, Switzerland: World Health Organization Publications.

Salepci, B., Caglayan, B., Kiral, N., Parmaksiz, T., Comert, S. S., Sarac, G., Fidan, A., \& Gungor, G. A. (2013). CPAP adherence of patients with obstructive sleep apnea. Respiratory Care, 58, 1467-1473. doi: 10.4187/respcare.02139

Sampaio, R., Pereira, M. G., \& Winck, J. C. (2012). Psychological morbidity, illness representations, and quality of life in female and male patients with obstructive sleep apnea syndrome. Psychology, Health \& Medicine, 17, 136-149. doi: 10.1080/13548506. 2011.579986

Sanford, S. D., Bush, A. J., Stone, K. C., Lichstein, K. L., \& Aguillard, N. (2008). Psychometric evaluation of the Beck Anxiety Inventory: A sample with sleep-disordered breathing. Behavioral Sleep Medicine, 6, 193-205. doi: 10.1080/15402000802162596

Saunamaki, T., \& Jehkonen, M. (2007). Depression and anxiety in obstructive sleep apnea syndrome: A review. Acta Neurologica Scandinavica, 116, 277-288. doi: 10.1111/j. 1600-0404.2007.00901.x

Sawyer, A. M., Canamucio, A., Moriarty, H., Weaver, T., Richards, K. C., \& Kuna, S. T. (2011). Do cognitive perceptions influence CPAP use? Patient Education and Counseling, 85, 85-91. doi: 10.1016./j.pec.2010.10.014

Sawyer, A. M., Deatrick, J. A., Kuna, S. T., \& Weaver, T. E. (2010). Differences in perceptions of the diagnosis and treatment of obstructive sleep apnea and continuous positive airway pressure therapy among adherers and nonadherers. Qualitative Health Research, 20, 873892. doi: $10.1177 / 1049732310365502$ 
Sawyer, A. M., Gooneratne, N. S., Marcus, C. L., Ofer, D., Richards, K. C., \& Weaver, T. (2011). A systematic review of CPAP adherence across age groups: Clinical and empiric insights for developing CPAP adherence interventions. Sleep Medicine Reviews, 15, 343356. doi: 10.1016/j.smrv.2011.01.003

Schwartz, A. R., Patil, S. P., Laffan, A. M., Polotsky, V., Schneider, H., \& Smith, P. L. (2008). Obesity and obstructive sleep apnea: Pathogenic mechanisms and therapeutic approaches. Proceedings of the American Thoracic Society, 5, 185-192. doi: 10.1513/pats.200708$137 \mathrm{MG}$

Shamian, J., \& Griffin, P. (2003). Translating research into health policy. Canadian Journal of Nursing Research, 35, 45-52.

Shamian, J., \& Shamian-Ellen, M. (2011). Shaping health policy: The role of nursing researchThree frameworks and their application to policy development. In A. S. Hinshaw \& P. A. Grady (Eds.), Shaping health policy through nursing research (pp. 35-51). New York, NY: Springer Publishing Company.

Shapiro, A. L. (2014). Anxiety in middle-aged men with obstructive sleep apnea: State of the science. Journal of the American Association of Nurse Practitioners, 26, 689-695. doi: $10.1002 / 2327-6924.12118$

Shapiro, A. L., Culp, S., \& Chertok, I. (2014). OSA symptoms associated with and predictive of anxiety in middle-aged men: Secondary analysis of NHANES data. Archives of Psychiatric Nursing, 28, 200-205. doi: 10.1016/j.apnu.2014.02.002

Shapiro, A. L., \& McCrone, S. (2016). CPAP nonadherence issues in a small sample of men with obstructive sleep apnea. Manuscript submitted for publication.

Shapiro, G. K., \& Shapiro, C. M. (2010). Factors that influence CPAP adherence: An overview. 
Sleep Breath, 14, 323-335. doi: 10.1007/s11325-010-0391-y

Simon, R. W., \& Nath, L. E. (2004). Gender and emotion in the United States: Do men and women differ in self-reports of feelings and expressive behavior? American Journal of Sociology, 109, 1137-1176.

Smith, C. E., Dauz, E., Clements, F., Werkowitch, M., \& Whitman, R. (2009). Patient education combined in a music and habit-forming intervention for adherence to continuous positive airway (CPAP) prescribed for sleep apnea. Patient Education and Counseling, 74, 184-190. doi: 10.1016/j.pec.2008.08.008

Smith, S., Lang, C., Sullivan, K., \& Warren, J. (2004a). Two new tools for assessing patients' knowledge and beliefs about obstructive sleep apnea and continuous positive airway pressure therapy. Sleep Medicine, 5, 359-367. doi: 10.1016/j.sleep.2003.12.007

Smith, S. S., Lang, C. P., Sullivan, K. A., \& Warren, J. (2004b). A preliminary investigation of the effectiveness of a sleep apnea education program. Journal of Psychosomatic Research, 56, 245-249. doi: 10.1016/S0022-3999(03)00545-2

Sparrow, D., Aloia, M. S., DeMolles, D. A., \& Gottlieb, D. J. (2010). A telemedicine intervention to improve adherence to continuous positive airway pressure: A randomised controlled trial. Thorax, 65, 1061-1066. doi:10.1136/thx.2009.133215

Stepnowsky, C. J., Palau, J. J., Gifford, A. L., \& Ancoli-Israel, S. (2007). A self-management approach to improving continuous positive airway pressure adherence and outcomes. Behavioral Sleep Medicine, 5, 131-146. doi: 10.108/15402000701190622

Stepnowsky, C., Zamora, T., Edwards, C., Liu, L., \& Agha, Z. (2013). Interventions to improve CPAP adherence and outcomes: Role of theory and behavioral change techniques. Journal of Sleep Disorders \& Therapy, 2, 1-11. doi: 10.4172/2167-0277.1000133 
Tabachnick, B. G., \& Fidell, L. S. (2007). Using multivariate statistics ( $5^{\text {th }}$ ed.). Boston, MA: Pearson Education, Inc.

Tarasiuk, A., \& Reuveni, H. (2013). The economic impact of obstructive sleep apnea. Current Opinion in Pulmonary Medicine, 19, 639-644. doi: 10.1097/MCP.0b013e3283659e1e

Trupp, R. J., Corwin, E. J., \& Ahijevych, K. L. (2011). The impact of educational message framing on adherence to continuous positive airway pressure therapy. Behavioral Sleep Medicine, 9, 38-52. doi: 10.1080/15402002.2011.533993

Tyrrell, J., Poulet, C., Pepin, J. -L., \& Veale, D. (2006). A preliminary study of psychological factors affecting patient's acceptance of CPAP therapy for sleep apnoea syndrome. Sleep Medicine, 7, 375-379. doi: 10.1016/j.sleep.2005.10.005

Tzischinsky, O., Shahrabani, S., \& Peled, R. (2011). Factors affecting the decision to be treated with continuous positive airway pressure for obstructive sleep apnea syndrome. IMAJ, 13, 413-419.

UCLA. (2000). UCLA stroke PROTECT report score card. Retrieved from http://strokeprotect. mednet.ucla.edu

Wang, W., He, G., Wang, M., Liu, L., \& Tang, H. (2012). Effects of patient education and progressive muscle relaxation alone or combined on adherence to continuous positive airway pressure treatment in obstructive sleep apnea patients. Sleep Breath, 16, 10491057. doi: 10.1007/s11325-011-0600-3

Ward, K., Hoare, K. J., \& Gott, M. (2014). What is known about the experiences of using CPAP for OSA from the users' perspective? A systematic integrative literature review. Sleep Medicine Reviews, 18, 357-366. doi: 10.1016/j.smrv.2014.01.001

Weaver, T. E., \& Sawyer, A. M. (2010). Adherence to continuous positive airway pressure 
treatment for obstructive sleep apnoea: Implications for future interventions. Indian Journal of Medical Research, 131, 245-258.

Weston, M. J., White, K. M., \& Peterson, C. A. (2013). Creating nursing's future: Translating research into evidence-based policy. Communicating Nursing Research, 46, 47-56.

White, K. M., Terry, D. J., Troup, C., Rempel, L. A., Norman, P., Mummery, K., ... Kenardy, J. (2012). An extended Theory of Planned Behavior intervention for older adults with type 2 diabetes and cardiovascular disease. Journal of Aging and Physical Activity, 20, 281299.

Whittemore, R. (2009). How can nursing intervention research reduce the research-practice gap? Canadian Journal of Nursing Research, 41(4), 7-15.

Wiese, H. J., Boethel, C., Phillips, B., Wilson, J. F., Peters, J., \& Viggiano, T. (2005). CPAP compliance: Video education may help! Sleep Medicine, 6, 171-174. doi: 10.1016/j. sleep.2004.08.006

Willman, M., Igelstrom, H., Martin, C., \& Asenlof, P. (2012). Experiences with CPAP treatment in patients with obstructive sleep apnea syndrome and obesity. Advances in Physiotherapy, 14, 166-174. doi: 10.3109/14038196.2012.704944

World Health Organization. (2010). Estimated obesity prevalence 2010. Retrieved from https://apps.who.int/infobase

Wozniak, D. R., Lasserson, T. J., \& Smith, I. (2014). Educational, supportive and behavioural interventions to improve usage of continuous positive airway pressure machines in adults with obstructive sleep apnea. The Cochrane Database of Systematic Reviews, 1, CD007736. doi:10.1002/14651858.CD007736.pub2

Ye, L., Liang, Z., \& Weaver, T. E. (2008). Predictors of health-related quality of life in patients 
with obstructive sleep apnoea. Journal of Advanced Nursing, 63, 54-63. doi: 10.1111/ j.1365-2648.2008.04652.x

Ye, L., Pack, A. I., Maislin, G., Dinges, D., Hurley, S., McCloskey, S., \& Weaver, T. E. (2012). Predictors of continuous positive airway pressure use during the first week of treatment. Journal of Sleep Research, 21, 419-426. doi: 10.1111/j.1365-2869.2011.00969.x

Young, T., Finn, L., Peppard, P. E., Szklo-Coxe, M., Austin, D., Nieto, J., ... Hla, K. M. (2008). Sleep disordered breathing and mortality: Eighteen-year follow-up of the Wisconsin Sleep Cohort. Sleep, 31, 1071-1078.

Young, T., Peppard, P. E., \& Taheri, S. (2005). Excess weight and sleep-disordered breathing. Journal of Applied Physiology, 99, 1592-1599. doi: 10.1152/japplphysiol.00587.2005. 


\section{Table 1}

\section{Tables}

Typologies of CPAP Adherers and Nonadherers Considered in the CPAP-SAVER Intervention Development

\begin{tabular}{ll} 
Adherent CPAP user & Nonadherent CPAP user \\
\hline Define risks associated with OSA & Unable to define risks associated with OSA \\
Identify outcome expectations from outset & Describe few outcomes expectations \\
Have fewer barriers than facilitators & Do not recognize own symptoms \\
$\begin{array}{l}\text { Facilitators less important later with treatment } \\
\text { use }\end{array}$ & $\begin{array}{l}\text { Describe barriers as more influential on CPAP } \\
\text { use than facilitators }\end{array}$ \\
$\begin{array}{l}\text { Develop and define goals and reasons for } \\
\text { CPAP use }\end{array}$ & $\begin{array}{l}\text { Facilitators of treatment absent or } \\
\text { unrecognized }\end{array}$ \\
$\begin{array}{l}\text { Describe positive belief in ability to use } \\
\text { CPAP even with potential or experienced } \\
\text { difficulties }\end{array}$ & $\begin{array}{l}\text { Describe low belief in ability to use CPAP } \\
\text { Proximate social influences prominent in } \\
\text { decisions to pursue diagnosis and treatment }\end{array}$ \\
$\begin{array}{l}\text { Describe early negative experiences with } \\
\text { CPAP, reinforcing low belief in ability to use } \\
\text { CPAP }\end{array}$ \\
$\begin{array}{l}\text { Unable to identify positive responses to } \\
\text { CPAP during early treatment }\end{array}$
\end{tabular}

From "Differences in Perceptions of the Diagnosis and Treatment of Obstructive Sleep Apnea and Continuous Positive Airway Pressure Therapy Among Adherers and Nonadherers," by A. M. Sawyer, J. A. Deatrick, S. T. Kuna, and T. E. Weaver, 2010, Qualitative Health Research, 20, p. 888. Copyright (C) 2010 by SAGE Publications. Reprinted with permission. 


\section{Table 2}

Abraham and Michie's (2008) Behavior Change Techniques Operationalized in the CPAPSAVER Intervention Study

\begin{tabular}{ll}
\hline Behavior Change Technique (Number) & CPAP-SAVER Intervention Component \\
\hline $\begin{array}{l}\text { Provide information about behavior-health } \\
\text { link (1) }\end{array}$ & Airway model, video, education sheet \\
Provide information on consequences (2) & Airway model, video, education sheet \\
$\begin{array}{l}\text { Provide information about others' approval } \\
(3)\end{array}$ & Support/Subjective norm calls \\
Prompt intention formation (4) & Support/Subjective norm calls \\
Provide general encouragement (6) & Support/Subjective norm calls \\
Provide feedback on performance (13) & Report card \\
Use follow-up prompts (18) & Support/Subjective norm calls \\
\hline
\end{tabular}

Adapted from "A Taxonomy of Behavior Change Techniques Used in Interventions," by C. Abraham \& S. Michie, 2008, Health Psychology, 27, p. 382. Copyright ( 2008 by American Psychological Association. Reprinted with permission. 
Table 3

Variables in the CPAP-SAVER Intervention Study

\begin{tabular}{|c|c|c|}
\hline Variable & Definition and Operationalization & Measurement/Instrument \\
\hline $\begin{array}{l}\text { CPAP Adherence } \\
\text { Behavior }\end{array}$ & $\begin{array}{l}\text { Consistent CPAP use, demonstrated by wearing mask/ } \\
\text { pillows connected to CPAP machine at the prescribed } \\
\text { pressure for at least } 4 \text { hours per night for } 70 \% \text { of the nights } \\
(7 \times 70 \%=5 \text { nights; } 30 \times 70 \%=21 \text { nights })\end{array}$ & $\begin{array}{l}\text { Smart card data (CPAP use } \\
\text { details, including AHI \& } \\
\text { mask-on time; adherence yes } \\
\text { or no) }\end{array}$ \\
\hline $\begin{array}{l}\text { CPAP Adherence } \\
\text { Intention }\end{array}$ & $\begin{array}{l}\text { Indication of how hard person is willing to try or how much } \\
\text { effort person is planning to exert to perform CPAP-adherence } \\
\text { behavior; person's motivation to act }\end{array}$ & $\begin{array}{l}\text { Intention Direct Measure } \\
\text { Questions (1-7; higher mean } \\
\text { indicates more positive } \\
\text { CPAP intention) }\end{array}$ \\
\hline $\begin{array}{l}\text { CPAP Adherence } \\
\text { Attitude }\end{array}$ & $\begin{array}{l}\text { Person's evaluation or appraisal of adhering to CPAP; } \\
\text { favorable (positive) or unfavorable (negative) }\end{array}$ & $\begin{array}{l}\text { Attitude Direct Measure } \\
\text { Questions (1-7; higher mean } \\
\text { indicates more positive } \\
\text { CPAP attitude) }\end{array}$ \\
\hline $\begin{array}{l}\text { CPAP Adherence } \\
\text { Subjective Norm }\end{array}$ & $\begin{array}{l}\text { Person's perceived social pressure to adhere or not adhere to } \\
\text { CPAP; belief that specific individuals (parents, spouse, close } \\
\text { friends, coworkers, or healthcare providers) approve or } \\
\text { disapprove of CPAP adherence }\end{array}$ & $\begin{array}{l}\text { Subjective Norm Direct } \\
\text { Measure Questions (1-7; } \\
\text { higher mean indicates more } \\
\text { CPAP social pressure) }\end{array}$ \\
\hline $\begin{array}{l}\text { CPAP Adherence } \\
\text { Perceived } \\
\text { Behavioral } \\
\text { Control }\end{array}$ & $\begin{array}{l}\text { Perception that person has/does not have capacity to adhere } \\
\text { to CPAP; includes self-efficacy and perceived controllability } \\
\text { to adhere to CPAP }\end{array}$ & $\begin{array}{l}\text { Perceived Behavioral } \\
\text { Control Direct Measure } \\
\text { Questions (1-7; higher mean } \\
\text { indicates higher CPAP } \\
\text { control perception) }\end{array}$ \\
\hline $\begin{array}{l}\text { CPAP Adherence } \\
\text { Beliefs }\end{array}$ & $\begin{array}{l}\text { Behavioral, normative, and control antecedents that lead to } \\
\text { the formation of a CPAP adherence attitude, subjective norm, } \\
\text { and perceived behavioral control, respectively }\end{array}$ & $\begin{array}{l}\text { Apnea Beliefs Scale ( } 24-120 \text {; } \\
\text { higher score indicates more } \\
\text { positive CPAP beliefs) }\end{array}$ \\
\hline $\begin{array}{l}\text { Background } \\
\text { Factors } \\
\text { (Demographics) }\end{array}$ & $\begin{array}{l}\text { Age (years) } \\
\text { Weight (pounds) and height (inches); BMI ( } \mathrm{m}^{2} \text {; calculated } \\
\text { from weight and height) } \\
\text { Gender (male or female) } \\
\text { Married (no or yes) or bed partner (no or yes) } \\
\text { Predominant ethnicity (White or other) } \\
\text { Highest educational level (Less than high school or high } \\
\text { school and beyond) } \\
\text { Socioeconomic status (income) and type of medical insurance } \\
\text { Employed (no or yes); work shift work (no or yes); work } \\
\text { night shift (no or yes) } \\
\text { OSA comorbidities (medical/car accident history) } \\
\text { Anxiety (score on Beck Anxiety Inventory) } \\
\text { OSA severity (apnea hypopnea index; mild = 5 to 15, } \\
\text { moderate = over } 15 \text { to } 30 \text {, and severe = over } 30 \text { ) } \\
\text { Oxygen saturation nadir (\%) } \\
\text { CPAP machine/mask make/model; humidification (no or yes) } \\
\text { CPAP pressure settings (centimeters of water) } \\
\text { Special CPAP settings, e.g. C-Flex (1, 2, or 3) }\end{array}$ & $\begin{array}{l}\text { Demographic survey } \\
\text { Beck Anxiety Inventory (0- } \\
63 ; 0-7=\text { minimal, } 8-15= \\
\text { mild, } 16-25=\text { moderate, and } \\
26-63=\text { severe }) \\
\text { OSA-CPAP Data Log (initial } \\
\text { sleep study results, CPAP } \\
\text { equipment and settings, and } \\
\text { CPAP smart card) }\end{array}$ \\
\hline
\end{tabular}




\section{Table 4}

Direct Questions for Measuring CPAP Adherence Attitude, CPAP Adherence Subjective Norm, CPAP Adherence Perceived Behavioral Control, and CPAP Adherence Intention in the CPAPSAVER Intervention Study

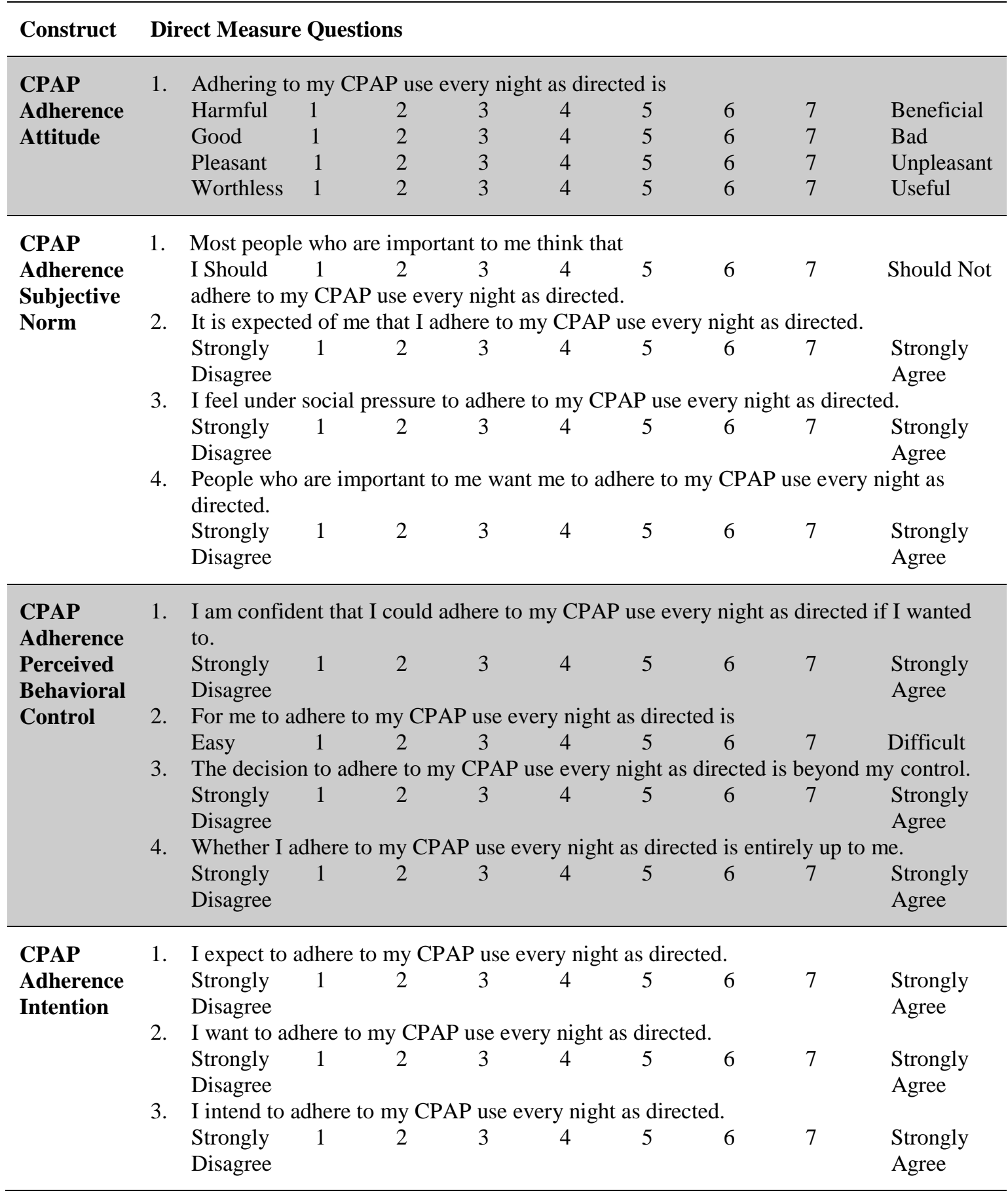

Note. Adapted from "Constructing Questionnaires Based on the Theory of Planned Behaviour: A Manual for Health Services Researchers" by J. J. Francis et al., 2004, Centre for Health Services Research. No permission needed to adapt Theory of Planned Behavior questionnaires. 
Table 5

CPAP-SAVER Intervention Study Protocol

\begin{tabular}{|c|c|c|c|c|}
\hline $\begin{array}{l}\text { Step } \\
\text { Number }\end{array}$ & Step & $\begin{array}{l}\text { Measures/ } \\
\text { Data Collected }\end{array}$ & Responsibility & $\begin{array}{l}\text { Participants: } \\
\text { Intervention, } \\
\text { Control, or } \\
\text { Both }\end{array}$ \\
\hline 1 & $\begin{array}{l}\text { Upon physician referral, } \\
\text { potential participant visits home } \\
\text { medical supplier (HMS) for } \\
\text { CPAP machine and training. }\end{array}$ & None & N/A & Both \\
\hline 2 & $\begin{array}{l}\text { Investigator (I) or respiratory } \\
\text { therapist (RT) gives potential } \\
\text { participant flyer about the study } \\
\text { and answers questions. }\end{array}$ & None & $\mathrm{I} / \mathrm{RT}$ & Both \\
\hline 3 & $\begin{array}{l}\text { I or RT reviews inclusion/ } \\
\text { exclusion criteria to ensure } \\
\text { participant's eligibility and } \\
\text { explains consent form with } \\
\text { potential participant. }\end{array}$ & $\begin{array}{l}\text { Consent } \\
\text { (Explanation) }\end{array}$ & $\mathrm{I} / \mathrm{RT}$ & Both \\
\hline 4 & $\begin{array}{l}\text { If participant agrees, I or RT } \\
\text { obtains informed consent \& } \\
\text { assigns participant a code for de- } \\
\text { identification (ID) purposes. } \\
\text { Participant is provided with this } \\
\text { number to be used throughout } \\
\text { study in place of his/her name. }\end{array}$ & $\begin{array}{l}\text { Consent (Signed } \\
\text { form) }\end{array}$ & I/RT & Both \\
\hline 5 & $\begin{array}{l}\text { Participant begins study: } \\
\text { a. } \text { RT pulls top envelope } \\
\text { before beginning CPAP } \\
\text { teaching. } \\
\text { b. Participant completes all } \\
\text { surveys inside envelope. } \\
\text { c. If sheet inside envelope } \\
\text { says Standard care, RT } \\
\text { proceeds with standard } \\
\text { care. } \quad \text { OR } \\
\text { If sheet inside envelope } \\
\text { says Intervention, RT } \\
\text { performs intervention } \\
\text { first: } \\
\text { i. RT displays airway } \\
\text { model as directed in } \\
\text { the script. } \\
\text { ii. RT shows video to } \\
\text { the participant. }\end{array}$ & $\begin{array}{l}\text { Demographic } \\
\text { survey } \\
\text { BAI, ABS, } \\
\text { TPB constructs } \\
\text { (CPAP attitude, } \\
\text { Subjective norm, } \\
\text { perceived } \\
\text { behavioral } \\
\text { control, } \\
\text { intention) }\end{array}$ & RT & Both \\
\hline
\end{tabular}




\begin{tabular}{|c|c|c|c|c|}
\hline & $\begin{array}{l}\text { iii. RT reviews The Risks } \\
\text { of Obstructive Sleep } \\
\text { Apnea \& the Benefits } \\
\text { of CPAP sheet with } \\
\text { participant following } \\
\text { script. Participant } \\
\text { keeps copy. } \\
\text { iv. RT initiates CPAP } \\
\text { Report Card (AHI \& } \\
\text { CPAP) \& reviews } \\
\text { with participant } \\
\text { following script. } \\
\text { Participant keeps } \\
\text { copy for updating. } \\
\text { v. RT reminds } \\
\text { participant that nurse } \\
\text { will call his/her home } \\
\text { at CPAP mid-week } \\
\text { one and CPAP mid- } \\
\text { week two. } \\
\text { vi. RT proceeds with } \\
\text { standard care. } \\
\text { d. RT discusses one-week } \\
\text { smart card reading. } \\
\text { RT arranges one-month } \\
\text { follow-up (f/u) visit. } \\
\text { f. RT gives participant } \\
\text { compensation \#1. }\end{array}$ & & & \\
\hline 6 & $\begin{array}{l}\text { RT contacts I to report: } \\
\text { a. Participant's ID number. } \\
\text { b. If participant is Standard } \\
\text { care or Intervention } \\
\text { group \& study start/end } \\
\text { dates } \\
\text { c. Date of CPAP } \\
\text { commencement } \\
\text { d. Participant's AHI/CPAP } \\
\text { settings from sleep study } \\
\text { e. Participant's CPAP set- } \\
\text { up: Machine type (make/ } \\
\text { model), mask type (make/ } \\
\text { model), CPAP setting in } \\
\text { cm H }{ }_{2} \mathrm{O}, \& \text { special } \\
\text { settings (ramp/C-Flex) } \\
\text { f. Two call dates \& } \\
\text { participant's phone }\end{array}$ & $\begin{array}{l}\text { Sleep study } \\
\text { results (AHI, } \\
\text { oxygen } \\
\text { saturation nadir) } \\
\text { CPAP machine/ } \\
\text { mask details } \\
\text { CPAP settings }\end{array}$ & I & Both \\
\hline
\end{tabular}




\begin{tabular}{|c|c|c|c|c|}
\hline & $\begin{array}{l}\text { number/alternate number } \\
\text { if in Intervention Group } \\
\text { g. Date/time of one-week } \\
\text { smart card reading } \\
\text { h. Date/time of one-month } \\
\text { f/u } \\
\text { i. I records all of the above } \\
\text { data onto calendar/log. }\end{array}$ & & & \\
\hline 7 & $\begin{array}{l}\text { CPAP mid-week one: I makes } \\
\text { support call \#1. See call log } \\
\text { (Appendix B) for specific } \\
\text { questions \& details. }\end{array}$ & $\begin{array}{l}\text { Responses to } \\
\text { support } \\
\text { questions (five } \\
\& \text { six) }\end{array}$ & $\mathrm{I}$ & Intervention \\
\hline 8 & $\begin{array}{l}\text { RT accesses smart card for one- } \\
\text { week data and updates report } \\
\text { card. }\end{array}$ & $\begin{array}{l}\text { Smart card data } \\
\text { (including AHI, } \\
\text { CPAP use, \& } \\
\text { adherence } \\
\text { measured by } \\
\text { mask-on time) } \\
\text { Changes in } \\
\text { CPAP/settings }\end{array}$ & RT & Both \\
\hline 9 & $\begin{array}{l}\text { I contacts HMS for info to } \\
\text { update CPAP Report Card. I } \\
\text { updates report card (AHI \& } \\
\text { CPAP) to review with participant } \\
\text { during support call \#2. }\end{array}$ & None & $\mathrm{I}$ & Intervention \\
\hline 10 & $\begin{array}{l}\text { CPAP week two: I makes } \\
\text { support call \#2, following same } \\
\text { questions as step } 8 \text { (see call log, } \\
\text { Appendix B). }\end{array}$ & $\begin{array}{l}\text { Responses to } \\
\text { support } \\
\text { questions (five } \\
\& \text { six) }\end{array}$ & I & Intervention \\
\hline 11 & $\begin{array}{l}\text { CPAP one month: Participant } \\
\text { visits HMS for f/u. } \\
\text { Intervention: Participant } \\
\text { completes measures (including } \\
\text { effectiveness survey), RT/I } \\
\text { updates report card, \& RT/I gives } \\
\text { participant thank you card \& } \\
\text { compensation \#2. } \\
\text { OR } \\
\text { Standard care: Participant } \\
\text { completes measures \& RT/I } \\
\text { gives participant thank you card } \\
\& \text { compensation \#2. }\end{array}$ & $\begin{array}{l}\text { BAI, ABS, } \\
\text { TPB constructs } \\
\text { (CPAP attitude, } \\
\text { subjective norm, } \\
\text { perceived } \\
\text { behavioral } \\
\text { control, } \\
\text { intention) } \\
\text { Smart card data } \\
\text { (including AHI, } \\
\text { CPAP use, \& } \\
\text { adherence } \\
\text { measured by } \\
\text { mask-on time) } \\
\text { CPAP settings }\end{array}$ & I & Both \\
\hline 12 & $\begin{array}{l}\text { Upon study completion, I visits } \\
\text { HMS offices to thank \& pay RT. }\end{array}$ & None & $\mathrm{I}$ & N/A \\
\hline
\end{tabular}




\section{Table 6}

Demographics of Participants in the CPAP-SAVER Intervention Study $(N=66)$

\begin{tabular}{|c|c|c|c|}
\hline Characteristic & $M / n$ & Range & $\%$ \\
\hline \multicolumn{4}{|l|}{ Age (Years) } \\
\hline Mean & 51.8 & \multirow[t]{2}{*}{$20-73$} & N/A \\
\hline $\mathrm{SD}$ & 13.1 & & N/A \\
\hline \multicolumn{4}{|l|}{ Weight (Pounds) } \\
\hline Mean & 232.8 & \multirow{2}{*}{$116-370$} & N/A \\
\hline SD & 58.5 & & N/A \\
\hline \multicolumn{4}{|l|}{ Height (Inches) } \\
\hline Mean & 67.6 & \multirow[t]{2}{*}{$58-76$} & N/A \\
\hline SD & 4.4 & & N/A \\
\hline \multicolumn{4}{|l|}{$\operatorname{BMI}\left(\mathrm{m}^{2}\right)$} \\
\hline Mean & 35.7 & \multirow[t]{2}{*}{$14.5-52.7$} & N/A \\
\hline $\mathrm{SD}$ & 8.0 & & N/A \\
\hline \multicolumn{4}{|l|}{ Gender } \\
\hline Male & 36 & \multirow[t]{2}{*}{ N/A } & 54.5 \\
\hline Female & 30 & & 45.5 \\
\hline \multicolumn{4}{|l|}{ Married } \\
\hline No & 22 & \multirow[t]{2}{*}{ N/A } & 33.3 \\
\hline Yes & 44 & & 66.7 \\
\hline \multicolumn{4}{|l|}{ Main Ethnicity } \\
\hline White & 64 & \multirow[t]{4}{*}{ N/A } & 97.0 \\
\hline Black & 0 & & 0.0 \\
\hline Hispanic & 2 & & 3.0 \\
\hline Other & 0 & & 10.0 \\
\hline \multicolumn{4}{|l|}{ Highest Educational Level } \\
\hline Grade School & 2 & \multirow[t]{3}{*}{ N/A } & 3.0 \\
\hline High School/GED/TASC & 23 & & 34.8 \\
\hline College/Technical & 41 & & 62.1 \\
\hline \multicolumn{4}{|l|}{ Annual Household Income } \\
\hline Less Than $\$ 20,000$ & 5 & \multirow[t]{3}{*}{ N/A } & 7.8 \\
\hline$\$ 20,000$ to $\$ 40,000$ & 14 & & 21.9 \\
\hline Higher Than $\$ 40,000$ & 45 & & 70.3 \\
\hline \multicolumn{4}{|l|}{ Medical Insurance Provider } \\
\hline Medicare & 11 & \multirow[t]{3}{*}{ N/A } & 16.9 \\
\hline Medicaid & 2 & & 3.1 \\
\hline Private/Other & 52 & & 80.0 \\
\hline \multicolumn{4}{|l|}{ Employed } \\
\hline No & 21 & \multirow[t]{2}{*}{ N/A } & 31.8 \\
\hline Yes & 45 & & 68.2 \\
\hline
\end{tabular}




\section{Table 7}

Sleep and OSA-Related Demographics of Participants in the CPAP-SAVER Intervention Study $(N=66)$

\begin{tabular}{|c|c|c|}
\hline Characteristic & $N$ & $\%$ \\
\hline \multicolumn{3}{|l|}{ OSA Severity (AHI) } \\
\hline Mild (5-15) & 29 & 43.9 \\
\hline Moderate (Over 15-30) & 16 & 24.3 \\
\hline Severe (Over 30) & 21 & 31.8 \\
\hline \multicolumn{3}{|l|}{ Work Shift Work } \\
\hline No & 54 & 81.8 \\
\hline Yes & 12 & 18.2 \\
\hline \multicolumn{3}{|l|}{ Work Night Shift } \\
\hline No & 59 & 89.4 \\
\hline Yes & 7 & 10.6 \\
\hline \multicolumn{3}{|l|}{ Bed Partner } \\
\hline No & 24 & 36.9 \\
\hline Yes & 41 & 63.1 \\
\hline \multicolumn{3}{|l|}{ OSA-Related Comorbidities } \\
\hline \multicolumn{3}{|l|}{ Atrial Fibrillation } \\
\hline No & 57 & 86.4 \\
\hline Yes & 9 & 13.6 \\
\hline \multicolumn{3}{|l|}{ Car Accident (as Driver) } \\
\hline No & 37 & 56.1 \\
\hline Yes & 29 & 43.9 \\
\hline \multicolumn{3}{|l|}{ Diabetes } \\
\hline No & 52 & 78.8 \\
\hline Yes & 14 & 21.2 \\
\hline \multicolumn{3}{|l|}{ Heart Attack } \\
\hline No & 61 & 92.4 \\
\hline Yes & 5 & 7.6 \\
\hline \multicolumn{3}{|l|}{ Heart Failure } \\
\hline No & 61 & 92.4 \\
\hline Yes & 5 & 7.6 \\
\hline \multicolumn{3}{|l|}{ High Blood Pressure } \\
\hline No & 25 & 37.9 \\
\hline Yes & 41 & 62.1 \\
\hline \multicolumn{3}{|l|}{ Stroke } \\
\hline No & 64 & 97.0 \\
\hline Yes & 2 & 3.0 \\
\hline
\end{tabular}




\section{Table 8}

CPAP-Related Characteristics of Participants in the CPAP-SAVER Intervention Study $(N=66)$

\begin{tabular}{lcc}
\hline Characteristic & $\boldsymbol{n}$ & $\boldsymbol{\%}$ \\
\hline CPAP Machine & & \\
Philips Respironics DreamStation & 52 & 78.8 \\
ResMed AirSense 10 & 9 & 13.6 \\
Philips Respironics REMStar & 4 & 6.1 \\
ResMed AirView & 1 & 1.5 \\
\hline C-Flex (Expiratory Pressure Relief) Setting & & \\
0 & 14 & 21.2 \\
1 & 2 & 3.0 \\
2 & 46 & 69.7 \\
3 & 4 & 6.1 \\
\hline Initial Mask Type & & \\
Full Face & 41 & 62.1 \\
Nasal & 13 & 19.7 \\
Nasal Pillows & 12 & 18.2 \\
\hline One-Month Mask Type & & \\
Full Face & 39 & 59.1 \\
Nasal & 14 & 21.2 \\
Nasal Pillows & 13 & 19.7 \\
\hline Meets Adherence Definition: One Week & & \\
Yes & 50 & 75.8 \\
No & 16 & 24.2 \\
\hline Meets Adherence Definition: One Month & 48 & 72.7 \\
Yes & 18 & 27.3 \\
No & & \\
\hline
\end{tabular}




\section{Table 9}

OSA- and CPAP-Related Descriptives for Participants in the CPAP-SAVER Intervention Study $(N=66)$

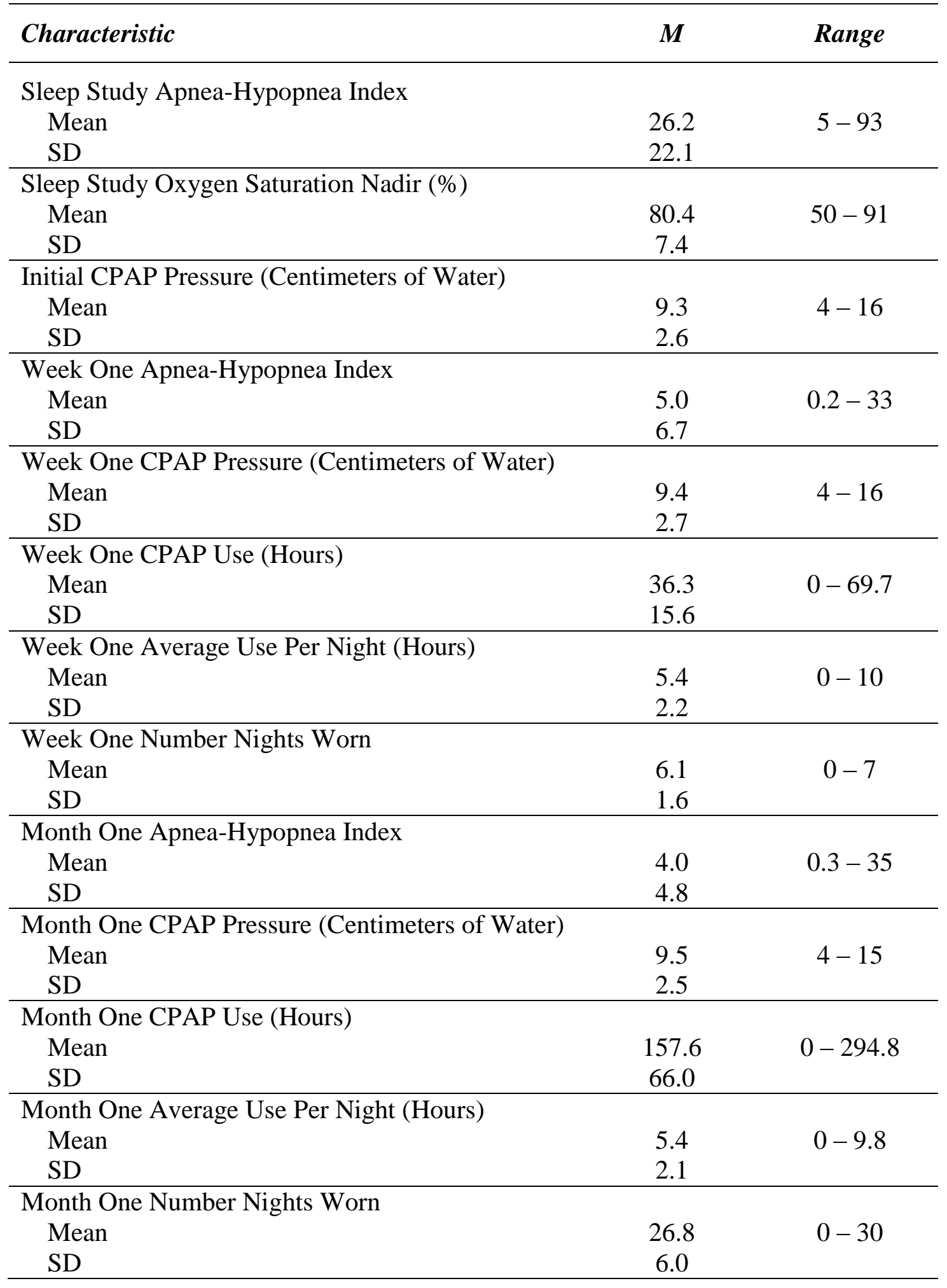




\section{Table 10}

Demographics of Participants in the CPAP-SAVER Intervention Study by Group

\begin{tabular}{|c|c|c|}
\hline Characteristic & Intervention & Standard Care \\
\hline \multicolumn{3}{|l|}{ Age (Years) } \\
\hline Mean & 53.0 & 50.7 \\
\hline $\mathrm{SD}$ & 12.9 & 13.5 \\
\hline \multicolumn{3}{|l|}{ Weight (Pounds) } \\
\hline Mean & 238.0 & 227.6 \\
\hline SD & 55.8 & 61.5 \\
\hline \multicolumn{3}{|l|}{ Height (Inches) } \\
\hline Mean & 68.0 & 67.2 \\
\hline $\mathrm{SD}$ & 4.7 & 4.2 \\
\hline \multicolumn{3}{|l|}{$\operatorname{BMI}\left(\mathrm{m}^{2}\right)$} \\
\hline Mean & 36.1 & 35.3 \\
\hline $\mathrm{SD}$ & 7.9 & 8.2 \\
\hline \multicolumn{3}{|l|}{ Gender } \\
\hline Male & 18 & 18 \\
\hline Female & 15 & 15 \\
\hline \multicolumn{3}{|l|}{ Married } \\
\hline No & 10 & 12 \\
\hline Yes & 23 & 21 \\
\hline \multicolumn{3}{|l|}{ Main Ethnicity } \\
\hline White & 32 & 32 \\
\hline Black & 0 & 0 \\
\hline Hispanic & 1 & 1 \\
\hline Other & 0 & 0 \\
\hline \multicolumn{3}{|l|}{ Highest Educational Level } \\
\hline Grade School & 0 & 2 \\
\hline High School/GED/TASC & 10 & 13 \\
\hline College/Technical & 23 & 18 \\
\hline \multicolumn{3}{|l|}{ Annual Household Income } \\
\hline Less Than $\$ 20,000$ & 3 & 2 \\
\hline$\$ 20,000$ to $\$ 40,000$ & 5 & 9 \\
\hline Higher Than $\$ 40,000$ & 25 & 20 \\
\hline \multicolumn{3}{|l|}{ Medical Insurance Provider } \\
\hline Medicare & 3 & 8 \\
\hline Medicaid & 2 & 0 \\
\hline Private/Other & 28 & 24 \\
\hline \multicolumn{3}{|l|}{ Employed } \\
\hline No & 8 & 13 \\
\hline Yes & 25 & 20 \\
\hline
\end{tabular}




\section{Table 11}

Sleep and OSA-Related Demographics of Participants in the CPAP-SAVER Intervention Study by Group

\begin{tabular}{|c|c|c|}
\hline Characteristic & Intervention & Standard Care \\
\hline \multicolumn{3}{|l|}{ OSA Severity (AHI) } \\
\hline Mild (5-15) & 16 & 13 \\
\hline Moderate (Over 15-30) & 5 & 11 \\
\hline Severe (Over 30) & 12 & 9 \\
\hline \multicolumn{3}{|l|}{ Work Shift Work } \\
\hline No & 26 & 28 \\
\hline Yes & 7 & 5 \\
\hline \multicolumn{3}{|l|}{ Work Night Shift } \\
\hline No & 29 & 30 \\
\hline Yes & 4 & 3 \\
\hline \multicolumn{3}{|l|}{ Bed Partner } \\
\hline No & 8 & 16 \\
\hline Yes & 25 & 16 \\
\hline \multicolumn{3}{|l|}{ OSA-Related Comorbidities } \\
\hline \multicolumn{3}{|l|}{ Atrial Fibrillation } \\
\hline No & 26 & 31 \\
\hline Yes & 7 & 2 \\
\hline \multicolumn{3}{|l|}{ Car Accident (as Driver) } \\
\hline No & 18 & 19 \\
\hline Yes & 15 & 14 \\
\hline \multicolumn{3}{|l|}{ Diabetes } \\
\hline No & 28 & 24 \\
\hline Yes & 5 & 9 \\
\hline \multicolumn{3}{|l|}{ Heart Attack } \\
\hline No & 32 & 29 \\
\hline Yes & 1 & 4 \\
\hline \multicolumn{3}{|l|}{ Heart Failure } \\
\hline No & 31 & 30 \\
\hline Yes & 2 & 3 \\
\hline \multicolumn{3}{|l|}{ High Blood Pressure } \\
\hline No & 13 & 12 \\
\hline Yes & 20 & 21 \\
\hline \multicolumn{3}{|l|}{ Stroke } \\
\hline No & 33 & 31 \\
\hline Yes & 0 & 2 \\
\hline
\end{tabular}




\section{Table 12}

CPAP-Related Characteristics of Participants in the CPAP-SAVER Intervention Study by Group

\begin{tabular}{lcc}
\hline Characteristic & Intervention & Standard Care \\
\hline CPAP Machine & 24 & 28 \\
Philips Respironics DreamStation & 4 & 5 \\
ResMed AirSense 10 & 4 & 0 \\
Philips Respironics REMStar & 1 & 0 \\
ResMed AirView & & 5 \\
C-Flex (Expiratory Pressure Relief) Setting & 9 & 1 \\
0 & 1 & 24 \\
1 & 22 & 3 \\
2 & 1 & 20 \\
3 & 21 & 7 \\
\hline Initial Mask Type & 6 & 6 \\
Full Face & 6 & 19 \\
Nasal & & 8 \\
Nasal Pillows & 20 & 6 \\
\hline One-Month Mask Type & 6 & 24 \\
Full Face & 7 & 9 \\
Nasal & & 25 \\
Nasal Pillows & 26 & \\
\hline Meets Adherence Definition: One Week & 7 & \\
Yes & 23 & \\
No & 10 & \\
\hline Meets Adherence Definition: One Month & & \\
Yes & & \\
No & 22 & \\
\hline
\end{tabular}




\section{Table 13}

OSA- and CPAP-Related Descriptives for Participants in the CPAP-SAVER Intervention Study by Group

\begin{tabular}{|c|c|c|}
\hline Characteristic & Intervention & Standard Care \\
\hline \multicolumn{3}{|c|}{ Sleep Study Apnea-Hypopnea Index } \\
\hline Mean & 28.7 & 23.7 \\
\hline SD & 26.3 & 16.9 \\
\hline \multicolumn{3}{|c|}{ Sleep Study Oxygen Saturation Nadir (\%) } \\
\hline Mean & 79.9 & 80.9 \\
\hline SD & 7.9 & 7.0 \\
\hline \multicolumn{3}{|c|}{ Initial CPAP Pressure (Centimeters of Water) } \\
\hline Mean & 9.3 & 9.2 \\
\hline SD & 2.6 & 2.6 \\
\hline \multicolumn{3}{|c|}{ Week One Apnea-Hypopnea Index } \\
\hline Mean & 4.7 & 5.3 \\
\hline $\mathrm{SD}$ & 6.1 & 7.4 \\
\hline \multicolumn{3}{|c|}{ Week One CPAP Pressure (Centimeters of Water) } \\
\hline Mean & 9.4 & 9.4 \\
\hline SD & 2.6 & 2.7 \\
\hline \multicolumn{3}{|c|}{ Week One CPAP Use (Hours) } \\
\hline Mean & 37.9 & 34.7 \\
\hline SD & 14.6 & 16.6 \\
\hline \multicolumn{3}{|c|}{ Week One Average Use Per Night (Hours) } \\
\hline Mean & 5.6 & 5.2 \\
\hline SD & 2.0 & 2.4 \\
\hline \multicolumn{3}{|c|}{ Week One Number Nights Worn } \\
\hline Mean & 6.3 & 5.9 \\
\hline SD & 1.1 & 2.0 \\
\hline \multicolumn{3}{|c|}{ Month One Apnea-Hypopnea Index } \\
\hline Mean & 3.4 & 4.5 \\
\hline $\mathrm{SD}$ & 3.3 & 6.1 \\
\hline \multicolumn{3}{|c|}{ Month One CPAP Pressure (Centimeters of Water) } \\
\hline Mean & 9.6 & 9.5 \\
\hline $\mathrm{SD}$ & 2.6 & 2.4 \\
\hline \multicolumn{3}{|c|}{ Month One CPAP Use (Hours) } \\
\hline Mean & 154.1 & 161.1 \\
\hline SD & 57.8 & 74.1 \\
\hline \multicolumn{3}{|c|}{ Month One Average Use Per Night (Hours) } \\
\hline Mean & 5.4 & 5.5 \\
\hline SD & 1.8 & 2.5 \\
\hline \multicolumn{3}{|c|}{ Month One Number Nights Worn } \\
\hline Mean & 27.1 & 26.6 \\
\hline SD & 3.4 & 7.8 \\
\hline
\end{tabular}




\section{Table 14}

Mean Instrument Scores for Participants in the CPAP-SAVER Intervention Study by Group

\begin{tabular}{|c|c|c|}
\hline Time & Intervention & Standard Care \\
\hline \multicolumn{3}{|c|}{ Pre Anxiety Score (Baseline) } \\
\hline Mean & 10.0 & 13.1 \\
\hline $\mathrm{SD}$ & 9.2 & 9.4 \\
\hline \multicolumn{3}{|c|}{ Post Anxiety Score (One Month) } \\
\hline Mean & 5.0 & 6.1 \\
\hline $\mathrm{SD}$ & 7.3 & 7.5 \\
\hline \multicolumn{3}{|c|}{ Pre Apnea Beliefs Score (Baseline) } \\
\hline Mean & 90.5 & 88.7 \\
\hline SD & 6.7 & 13.0 \\
\hline \multicolumn{3}{|c|}{ Post Apnea Beliefs Score (One Month) } \\
\hline Mean & 94.3 & 87.2 \\
\hline SD & 12.2 & 12.1 \\
\hline \multicolumn{3}{|c|}{ Pre Attitude Score (Baseline) } \\
\hline Mean & 5.6 & 5.6 \\
\hline SD & 1.1 & 1.0 \\
\hline \multicolumn{3}{|c|}{ Post Attitude Score (One Month) } \\
\hline Mean & 6.1 & 5.6 \\
\hline SD & 1.0 & 1.0 \\
\hline \multicolumn{3}{|c|}{ Pre Subjective Norm Score Baseline) } \\
\hline Mean & 5.4 & 5.4 \\
\hline SD & 0.8 & 0.9 \\
\hline \multicolumn{3}{|c|}{ Post Subjective Norm Score (One Month) } \\
\hline Mean & 5.4 & 5.6 \\
\hline SD & 1.1 & 0.9 \\
\hline \multicolumn{3}{|c|}{ Pre Perceived Behavioral Control Score (Baseline) } \\
\hline Mean & 4.9 & 4.9 \\
\hline SD & 0.7 & 0.8 \\
\hline \multicolumn{3}{|c|}{ Post Perceived Behavioral Control Score (One Month) } \\
\hline Mean & 4.8 & 4.8 \\
\hline SD & 0.8 & 0.7 \\
\hline \multicolumn{3}{|c|}{ Pre Intention Score (Baseline) } \\
\hline Mean & 6.6 & 6.7 \\
\hline SD & 0.6 & 0.7 \\
\hline \multicolumn{3}{|c|}{ Post Intention Score (One Month) } \\
\hline Mean & 6.3 & 6.1 \\
\hline SD & 1.1 & 1.1 \\
\hline
\end{tabular}




\section{Table 15}

OSA- and CPAP-Related Descriptives for Participants in the CPAP-SAVER Intervention Study by Gender

\begin{tabular}{|c|c|c|}
\hline Characteristic & Male & Female \\
\hline \multicolumn{3}{|c|}{ Sleep Study Apnea-Hypopnea Index } \\
\hline Mean & 29.6 & 22.8 \\
\hline SD & 22.0 & 22.1 \\
\hline \multicolumn{3}{|c|}{ Sleep Study Oxygen Saturation Nadir (\%) } \\
\hline Mean & 81.1 & 79.6 \\
\hline SD & 6.4 & 8.5 \\
\hline \multicolumn{3}{|c|}{ Initial CPAP Pressure (Centimeters of Water) } \\
\hline Mean & 9.8 & 8.7 \\
\hline $\mathrm{SD}$ & 2.5 & 2.7 \\
\hline \multicolumn{3}{|c|}{ Week One Apnea-Hypopnea Index } \\
\hline Mean & 4.9 & 5.1 \\
\hline $\mathrm{SD}$ & 6.6 & 6.9 \\
\hline \multicolumn{3}{|c|}{ Week One CPAP Pressure (Centimeters of Water) } \\
\hline Mean & 9.8 & 8.9 \\
\hline $\mathrm{SD}$ & 2.5 & 2.8 \\
\hline \multicolumn{3}{|c|}{ Week One CPAP Use (Hours) } \\
\hline Mean & 37.2 & 35.3 \\
\hline $\mathrm{SD}$ & 15.3 & 16.2 \\
\hline \multicolumn{3}{|c|}{ Week One Average Use Per Night (Hours) } \\
\hline Mean & 5.6 & 5.1 \\
\hline $\mathrm{SD}$ & 2.1 & 2.3 \\
\hline \multicolumn{3}{|c|}{ Week One Number Nights Worn } \\
\hline Mean & 6.3 & 5.9 \\
\hline $\mathrm{SD}$ & 1.3 & 1.9 \\
\hline \multicolumn{3}{|c|}{ Month One Apnea-Hypopnea Index } \\
\hline Mean & 3.4 & 4.7 \\
\hline $\mathrm{SD}$ & 2.8 & 6.6 \\
\hline \multicolumn{3}{|c|}{ Month One CPAP Pressure (Centimeters of Water) } \\
\hline Mean & 9.9 & 9.0 \\
\hline SD & 2.4 & 2.5 \\
\hline \multicolumn{3}{|c|}{ Month One CPAP Use (Hours) } \\
\hline Mean & 161.8 & 152.7 \\
\hline SD & 61.3 & 72.0 \\
\hline \multicolumn{3}{|c|}{ Month One Average Use Per Night (Hours) } \\
\hline Mean & 5.6 & 5.3 \\
\hline SD & 2.0 & 2.4 \\
\hline \multicolumn{3}{|c|}{ Month One Number Nights Worn } \\
\hline Mean & 28.0 & 25.4 \\
\hline $\mathrm{SD}$ & 3.6 & 7.8 \\
\hline
\end{tabular}




\section{Table 16}

Mean Instrument Scores for Participants in the CPAP-SAVER Intervention Study by Gender

\begin{tabular}{|c|c|c|}
\hline Time & Male & Female \\
\hline \multicolumn{3}{|c|}{ Pre Anxiety Score (Baseline) } \\
\hline Mean & 9.0 & 14.6 \\
\hline SD & 9.3 & 8.6 \\
\hline \multicolumn{3}{|c|}{ Post Anxiety Score (One Month) } \\
\hline Mean & 3.7 & 7.9 \\
\hline SD & 5.6 & 8.8 \\
\hline \multicolumn{3}{|c|}{ Pre Apnea Beliefs Score (Baseline) } \\
\hline Mean & 89.3 & 90.1 \\
\hline SD & 10.3 & 10.5 \\
\hline \multicolumn{3}{|c|}{ Post Apnea Beliefs Score (One Month) } \\
\hline Mean & 88.0 & 94.0 \\
\hline $\mathrm{SD}$ & 13.1 & 11.3 \\
\hline \multicolumn{3}{|c|}{ Pre Attitude Score (Baseline) } \\
\hline Mean & 5.6 & 5.6 \\
\hline $\mathrm{SD}$ & 1.1 & 1.0 \\
\hline \multicolumn{3}{|c|}{ Post Attitude Score (One Month) } \\
\hline Mean & 5.6 & 6.1 \\
\hline SD & 1.1 & 0.9 \\
\hline \multicolumn{3}{|c|}{ Pre Subjective Norm Score (Baseline) } \\
\hline Mean & 5.5 & 5.4 \\
\hline $\mathrm{SD}$ & 0.7 & 1.0 \\
\hline \multicolumn{3}{|c|}{ Post Subjective Norm Score (One Month) } \\
\hline Mean & 5.6 & 5.5 \\
\hline SD & 1.1 & 0.8 \\
\hline \multicolumn{3}{|c|}{ Pre Perceived Behavioral Control Score (Baseline) } \\
\hline Mean & 4.9 & 4.9 \\
\hline $\mathrm{SD}$ & 0.8 & 0.7 \\
\hline \multicolumn{3}{|c|}{ Post Perceived Behavioral Control Score (One Month) } \\
\hline Mean & 4.7 & 5.0 \\
\hline SD & 0.8 & 0.7 \\
\hline \multicolumn{3}{|c|}{ Pre Intention Score (Baseline) } \\
\hline Mean & 6.6 & 6.7 \\
\hline SD & 0.7 & 0.6 \\
\hline \multicolumn{3}{|c|}{ Post Intention Score (One Month) } \\
\hline Mean & 6.0 & 6.5 \\
\hline SD & 1.2 & 0.8 \\
\hline
\end{tabular}




\section{Table 17}

Frequencies of Report Card Grades for Intervention Group Participants in the CPAP-SAVER Study

\begin{tabular}{lcc}
\hline Report Card Grade & One Week & One Month \\
\hline Grade Given by Participant & & \\
A & 14 & 15 \\
B & 16 & 8 \\
C & 1 & 7 \\
Nonadherent & 2 & 3 \\
\hline Grade Given by Respiratory Therapist & & \\
A & 15 & 16 \\
B & 8 & 6 \\
C & 6 & 4 \\
Nonadherent & 4 & 7 \\
\hline
\end{tabular}


Table 18

Intervention Effectiveness Survey Results: Mean Scores for the CPAP-SAVER Study Components

\begin{tabular}{lcccc}
\hline CPAP-SA VER Study Component & Helpful & Liked & Understood & Motivated \\
\hline Support Calls & & & & \\
$\quad$ Mean & 3.4 & 3.4 & 3.8 & 3.2 \\
$\quad$ SD & 0.9 & 0.9 & 0.5 & 1.3 \\
\hline Airway Model & & & & \\
$\quad$ Mean & 3.6 & 3.2 & 3.8 & 3.2 \\
$\quad$ SD & 0.5 & 1.1 & 0.4 & 1.2 \\
\hline Video & & & & \\
Mean & 3.5 & 3.1 & 3.7 & 3.2 \\
SD & 0.7 & 1.0 & 0.5 & 1.2 \\
\hline Education Sheet & & & & 3.1 \\
Mean & 3.5 & 3.3 & 3.6 & 1.1 \\
$\quad$ SD & 0.6 & 0.9 & 0.6 & 3.2 \\
\hline Report Card & & & & 1.0 \\
$\quad$ Mean & 3.4 & 3.4 & 3.6 & \\
$\quad$ SD & 0.7 & 0.8 & 0.7 & \\
\hline
\end{tabular}




\section{Figures}

\section{Figure 1}

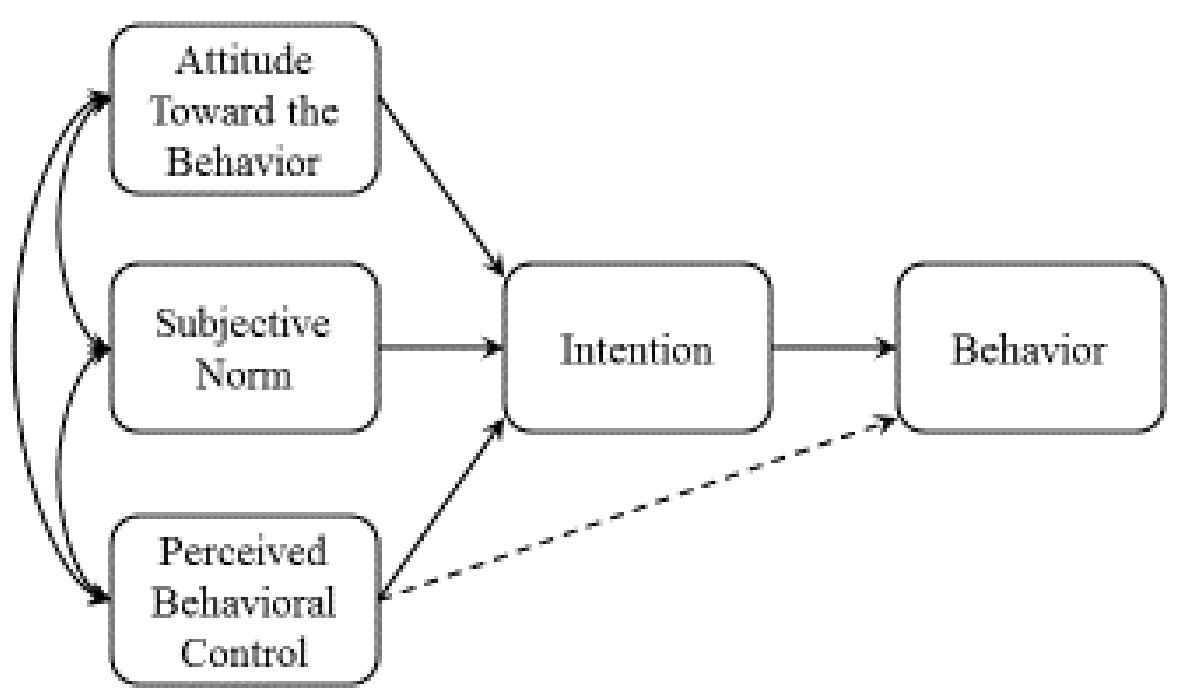

Figure 1. Theory of Planned Behavior. From Attitudes, Personality and Behavior ( $2^{\text {nd }}$ ed., p. 118), by I. Ajzen, 2005, Berkshire, England: Open University Press. Copyright (C 2005 by Open University Press. Reproduced with the kind permission of Open University Press. All rights reserved. 
Figure 2

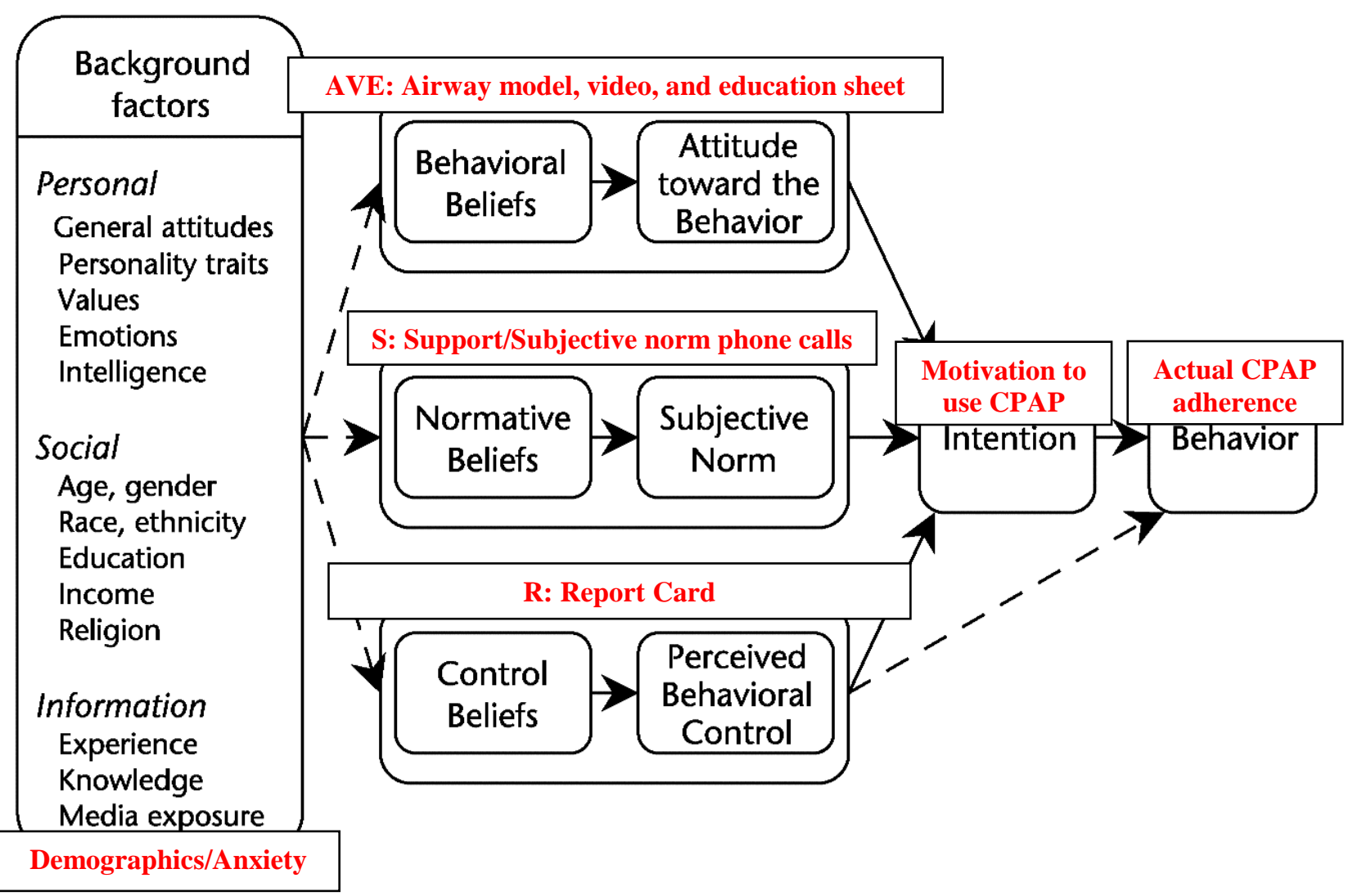

Figure 2. CPAP-SAVER intervention (in overlaid boxes with red text) to promote CPAP adherence using the Theory of Planned Behavior as the guiding framework. Adapted from Attitudes, Personality and Behavior ( $2^{\text {nd }}$ ed., p. 135), by I. Ajzen, 2005, Berkshire, England: Open University Press. Copyright (C 2005 by Open University Press. Reproduced with the kind permission of Open University Press. All rights reserved. 


\section{Figure 3}

$\mathbf{z}$ tests - Proportions: Difference between two independent proportions

Analysis: A priori: Compute required sample size

Input:

Tail(s)

Proportion $\mathrm{p} 2$

$=$ One

Proportion $\mathrm{p} 1$

$=0.6$

$\alpha$ err prob

$=0.3$

Power (1- $\beta$ err prob)

$=0.05$

Allocation ratio N2/N1

$=0.8$

$=1$

Output: Critical z

Sample size group 1

$=1.6448536$

Sample size group 2

$=33$

$=33$

Total sample size

$=66$

Actual power

$=0.8006400$

Figure 3. Using G*Power 3.1 (Faul, Erdfelder, Lang, \& Buchner, 2007), an a priori power analysis was conducted to determine sample size. CPAP adherence is reported to be $30-60 \%$;

proportions $\mathrm{p} 1$ and $\mathrm{p} 2$ were determined using this data. Note that $\mathrm{p} 1=$ proportion of adherence in the control group and $\mathrm{p} 2=$ proportion of adherence in the intervention group. 


\section{Appendices}

\section{Appendix A}

Participant Identification Number

Date

Instructions: Please answer the following questions to provide us basic information about you and your life circumstances.

1. What is your current age in years?

2. What is your current weight in pounds?

3. What is your current height in feet/inches?

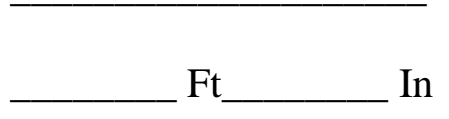

4. What is your gender? Please circle:

Male Female

5. Are you married? Please circle:

No Yes

6. What is your main ethnicity? Please circle:

White Black

Hispanic Other

7. What is your highest education level? Please circle:

Grade School

High School/GED/TASC

College/Technical

8. What is your total annual household income? Please circle: Less than $\$ 20,000$

$\$ 20,000$ to $\$ 40,000$

Higher than $\$ 40,000$

9. Who is your medical insurance provider? Please list:

10. Are you employed? Please circle:

$\begin{array}{ll}\text { No } & \text { Yes } \\ \text { No } & \text { Yes } \\ \text { No } & \text { Yes }\end{array}$

12. Do you work night shift? Please circle:

\section{Continued next page...}


Participant Identification Number

13. Do you have a bed partner? Please circle: (Person sleeping in the same bed with you regularly)
Date

Yes

14. Have you had (or do you now have) any of the following conditions?

$\begin{array}{llr}\text { Atrial fibrillation } & \text { No } & \text { Yes } \\ \text { Car accident (as the driver) } & \text { No } & \text { Yes } \\ \text { Diabetes } & \text { No } & \text { Yes } \\ \text { Heart attack } & \text { No } & \text { Yes } \\ \text { Heart failure } & \text { No } & \text { Yes } \\ \text { High blood pressure } & \text { No } & \text { Yes } \\ \text { Stroke } & \text { No } & \text { Yes }\end{array}$

Continued next page... 
Participant Identification Number

Date

NOTE: Administered the official, purchased version of the Beck Anxiety Inventory; this is an unofficial copy from the internet.

Instructions: Indicate how much you have been bothered by each symptom during the past week, including today, by circling the number in the column that most closely corresponds to how you've been feeling.

\begin{tabular}{|c|c|c|c|}
\hline NOT & MILDLY & MODERATELY & SEVERELY \\
AT & It did not & It was very & I could barely \\
ALL & $\begin{array}{c}\text { bother me } \\
\text { much. }\end{array}$ & $\begin{array}{c}\text { unpleasant but I } \\
\text { could stand it. }\end{array}$ & stand it. \\
&
\end{tabular}

\begin{tabular}{|c|c|c|c|c|}
\hline Numbness or tingling & 0 & 1 & 2 & 3 \\
\hline Feeling hot & 0 & 1 & 2 & 3 \\
\hline Wobbliness in legs & 0 & 1 & 2 & 3 \\
\hline Unable to relax & 0 & 1 & 2 & 3 \\
\hline Fear of the worst happening & 0 & 1 & 2 & 3 \\
\hline Dizzy or lightheaded & 0 & 1 & 2 & 3 \\
\hline Heart pounding or racing & 0 & 1 & 2 & 3 \\
\hline Unsteady & 0 & 1 & 2 & 3 \\
\hline Terrified & 0 & 1 & 2 & 3 \\
\hline Nervous & 0 & 1 & 2 & 3 \\
\hline Feelings of choking & 0 & 1 & 2 & 3 \\
\hline Hands trembling & 0 & 1 & 2 & 3 \\
\hline Shaky & 0 & 1 & 2 & 3 \\
\hline Fear of losing control & 0 & 1 & 2 & 3 \\
\hline Difficulty breathing & 0 & 1 & 2 & 3 \\
\hline Fear of dying & 0 & 1 & 2 & 3 \\
\hline Scared & 0 & 1 & 2 & 3 \\
\hline $\begin{array}{l}\text { Indigestion or discomfort in } \\
\text { abdomen }\end{array}$ & 0 & 1 & 2 & 3 \\
\hline Faint & 0 & 1 & 2 & 3 \\
\hline Face flushed & 0 & 1 & 2 & 3 \\
\hline Sweating (not due to heat) & 0 & 1 & 2 & 3 \\
\hline
\end{tabular}

Continued next page... 
Participant Identification Number

Date

Instructions: The next questions in this survey make use of rating scales with $\mathbf{7}$ choices; you are to circle the number that best describes your opinion. For example, if you were asked to rate "The Weather in Florida" on such a scale, the 7 choices should be interpreted as follows:

The Weather in Florida is:

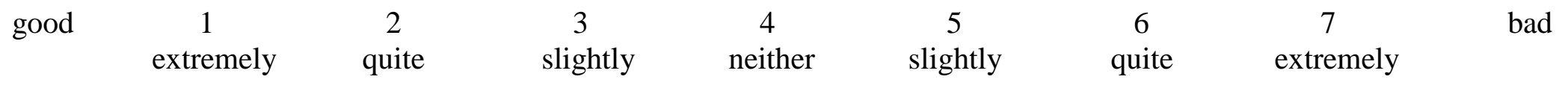

If you think the weather in Florida is extremely good, then you would circle the number 1, as follows:

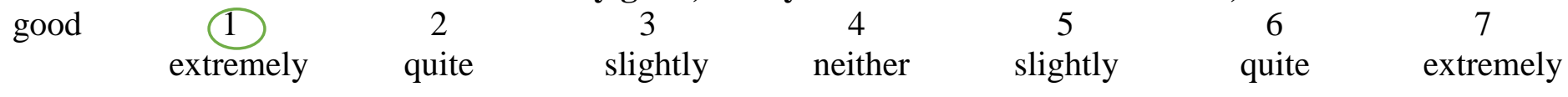

If you think the weather in Florida is quite bad, then you would circle the number 6, as follows.

good

extremely
2

quite
3
slightly
4
neither
5
slightly
6
7

extremely 
Participant Identification Number

Date

Each of the following questions relates to adhering to your CPAP use every night. Using the instructions from page one, please circle the number to represent your response to each question. Please answer every question. Please only circle one response for each question.

1. Adhering to my CPAP use every night as directed is

$\begin{array}{lllll}\text { harmful } & 1 & 2 & 3 & 4\end{array}$

4

5

6

7 beneficial

2. Adhering to my CPAP use every night as directed is good 1

2

3

4

5

6

$7 \quad$ bad

3. Adhering to my CPAP use every night as directed is $\begin{array}{llll}\text { pleasant } & 1 & 2 & 3\end{array}$

$4 \quad 5$

$5 \quad 6$

$\begin{array}{lll}6 & 7 & \text { unpleasant }\end{array}$

4. Adhering to my CPAP use every night as directed is worthless

2

3

4

5

6

$7 \quad$ useful

5. Most people who are important to me think that

$\begin{array}{llll}\text { I should } & 1 & 2 & 3\end{array}$

4

5

6

$7 \quad$ should not

adhere to my CPAP use every night as directed.

Continued next page... 
Participant Identification Number

Date

Each of the following questions relates to adhering to your CPAP use every night. Using the instructions from page one, please circle the number to represent your response to each question. Please answer every question. Please only circle one response for each question.

6. It is expected of me that I adhere to my CPAP use every night as directed.

$\begin{array}{lllllllll}\begin{array}{l}\text { strongly } \\ \text { disagree }\end{array} & 1 & 2 & 3 & 4 & 5 & 6 & 7 & \text { strongly } \\ \text { agree }\end{array}$

7. I feel under social pressure to adhere to my CPAP use every night as directed.

$\begin{array}{lllllllll}\begin{array}{l}\text { strongly } \\ \text { disagree }\end{array} & 1 & 2 & 3 & 4 & 5 & 6 & 7 \text { strongly } \\ \text { agree }\end{array}$

8. People who are important to me want me to adhere to my CPAP use every night as directed.

$\begin{array}{llllllll}\begin{array}{l}\text { strongly } \\ \text { disagree }\end{array} & 1 & 2 & 3 & 4 & 5 & 6 & 7 \text { strongly } \\ \text { agree }\end{array}$

9. I am confident that I could adhere to my CPAP use every night as directed if I wanted to.

$\begin{array}{llllllll}\begin{array}{l}\text { strongly } \\ \text { disagree }\end{array} & 1 & 2 & 3 & 4 & 5 & 6 & 7 \\ \text { agree }\end{array}$

10. For me to adhere to my CPAP use every night as directed is
easy
2
34
5
6
$7 \quad$ difficult

Continued next page... 
Participant Identification Number

Date

Each of the following questions relates to adhering to your CPAP use every night. Using the instructions from page one, please circle the number to represent your response to each question. Please answer every question. Please only circle one response for each question.

11. The decision to adhere to my CPAP use every night as directed is beyond my control.

$\begin{array}{lllllllll}\begin{array}{l}\text { strongly } \\ \text { disagree }\end{array} & 1 & 2 & 3 & 4 & 5 & 6 & 7 & \text { strongly } \\ \text { agree }\end{array}$

12. Whether I adhere to my CPAP use every night as directed is entirely up to me.

$\begin{array}{llllllll}\begin{array}{l}\text { strongly } \\ \text { disagree }\end{array} & 1 & 2 & 3 & 4 & 5 & 6 & 7 \text { strongly } \\ \text { agree }\end{array}$

13. I expect to adhere to my CPAP use every night as directed.

$\begin{array}{llllllll}\begin{array}{l}\text { strongly } \\ \text { disagree }\end{array} & 1 & 2 & 3 & 4 & 5 & 6 & 7 \text { strongly } \\ \text { agree }\end{array}$

14. I want to adhere to my CPAP use every night as directed.

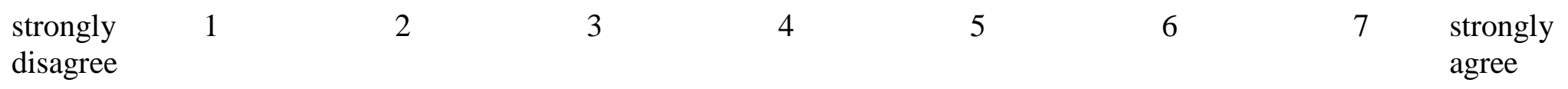

15. I intend to adhere to my CPAP use every night as directed.

$\begin{array}{llllllll}\begin{array}{l}\text { strongly } \\ \text { disagree }\end{array} & 1 & 2 & 3 & 4 & 5 & 6 & 7 \text { strongly } \\ \text { agree }\end{array}$

Continued Next Page... 
Participant Identification Number

Date

Instructions: Answer each of these questions by shading the number that best represents your answer.
(1)
(2)
(3)
(4)
(5)

Strongly disagree

Disagree Not sure/Neutral

Agree

Strongly agree

Sleep apnea has no effect on my life

(1) (2) (3) (4) (5)

If things become too much I generally don't go through with them

(1) (2) (3) (4) (5)

CPAP is "the answer" to my sleep apnea

(1) (2) (3) (4) (5)

Sleep apnea gets in the way of my friendships

(1) (2) (3) (4) (5)

I intend to use the CPAP machine all night every night.

(1) (2) (3) (4) (5)

I believe using the CPAP mask will be a nuisance

(1) (2) (3) (4) (5)

I am willing to ask for help when it is required

(1) (2) (3) (4) (5)

CPAP is the best treatment for my health problems

(1) (2) (3) (4) (5)

I am willing to follow the directions of medical staff "to the letter"

(1) (2) (3) (4) (5)

I believe that using CPAP is very confusing

(1) (2) (3) (4) (5)

Wearing the CPAP mask will make falling asleep hard

(1) (2) (3) (4) (5)

Once I make a decision, I stick with that decision

(1) (2) (3) (4) (5)

Wearing the CPAP mask will improve the quality of my sleep

(1) (2) (3) (4) (5)

I find it stressful to use new machinery or technology

(1) (2) (3) (4) (5)

Good health is secondary to being able to do what I want in life

(1) (2) (3) (4) (5)

I enjoy trying new things, like snorkeling

(1) (2) (3) (4) (5)

I don't believe I have a sleep problem

(1) (2) (3) (4) (5)

I find it embarrassing to ask for help

(1) (2) (3) (4) (5)

Sleep apnea is my major health problem

(1) (2) (3) (4) (5)

I believe that CPAP will make little difference to my sleep

(1) (2) (3) (4) (5)

I want to improve my health

(1) (2) (3) (4) (5)

I am confident that I will be able to use the CPAP machine as taught

(1) (2) (3) (4) (5)

I would try anything that I thought might help my sleep apnea

(1) (2) (3) (4) (5)

I believe that I know what is the best treatment for me

(1) (2) (3) (4) (5)

\section{Thank you!}




\section{Appendix B}

Participant ID\# Call \#1 \#2 Date

\section{Support/Subjective Norm Call Log}

1. Greeting: Hello, Mr./Mrs. , this is April Shapiro, the nurse doing the CPAP study. I am calling to check up on you, but first want to reconfirm: Are you still volunteering to participate in the study? Yes? Proceed. No? Thank person and inform him/her that all data collected to this point will be destroyed by shredding, including the consent form and questionnaires. Remind person that withdrawing from study does not affect treatment.

2. Is this a good time for you to talk? I have just a few questions I wanted to ask you about your sleep apnea and CPAP. It will only take about 5 minutes or less.

Yes? Proceed. No? Day/Time Best to Call Back

3. How are you doing? Are you having any machine or mask problems?

4. Let's talk about your CPAP Report Card number called the AHI (apnea-hypopnea index).

A. This number represents the number of times you have slowed or no breathing per hour through the night; the slowed breaths are hypopneas and no breaths are apneas.

B. Explain most recent AHI/severity rating: 5-15 mild, over 15-30 moderate, over 30 severe

C. CPAP is the best treatment for sleep apnea and will help improve your number. As you wear your CPAP, the number should go lower and lower, meaning your sleep apnea is getting better and better. As long as you wear your CPAP to sleep, your sleep apnea should remain under control. Treating your sleep apnea by wearing your CPAP will also decrease your risk of a heart attack, stroke, and falling asleep while driving or working.

D. Let's review your other report card numbers.

E. Do you have any questions about your numbers and what they mean?

5. A. What symptom of your sleep apnea has been the most troublesome to you?

\begin{tabular}{lll} 
Witnessed apneas & Snoring & Gasping/Choking at night \\
Excessive sleepiness & Nonrefreshing sleep & Sleep fragmentation \\
Nocturia & Morning headaches & Decreased concentration \\
Memory loss & Decreased libido & Irritability \\
Other & & \\
\hline
\end{tabular}

B. Do you see improvement in that symptom since using your CPAP? No Yes

6. A. Do you believe it is important to wear your CPAP every night? No Yes

B. Does your family or those people closest to you agree?

C. Are they supportive of your decision to treat your sleep apnea?

No Yes

D. Are they supportive of your decision to wear CPAP?

No Yes

No Yes

7. I'm pleased you took the initiative to get treatment for your sleep apnea. It's important to use CPAP every night, to cut down on your health risks and to improve your quality of life.

8. Do you have any questions or comments for me?

9. Well, thank you for your time. I'll call you one more time to check in. When I call next week, is there a day/time better for you? Yes? Day and time Okay; I'll talk to you next week. If you have any CPAP issues, give a call. 


\section{Appendix C}

\section{The Risks of Obstructive Sleep Apnea \& the Benefits of CPAP}

Obstructive apneas happen when you are asleep and your tongue and palate relax too much. When air can't get into your lungs, you stop breathing and your brain triggers you to wake up to take a breath. These apneas are dangerous to your health and can happen hundreds of times a night! Did you know that CPAP can eliminate the apneas? In order for this to happen, CPAP must be worn as directed, every time you sleep.

\section{What are the risks associated with}

\section{untreated obstructive sleep apnea?}

- High blood pressure

- Heart disease \& stroke

- Diabetes

- Falling asleep while driving or working, leading to accidents

- Long-term effects of low oxygen \& high carbon dioxide levels, which can affect thinking, behavior, \& mood

\section{What are the benefits of CPAP?}

- Improved blood pressure

- Decreased heart attack \& stroke risk

- Improved blood sugar levels

- More restful sleep

- Increased daytime energy \& alertness

- Improved mood \& relationships
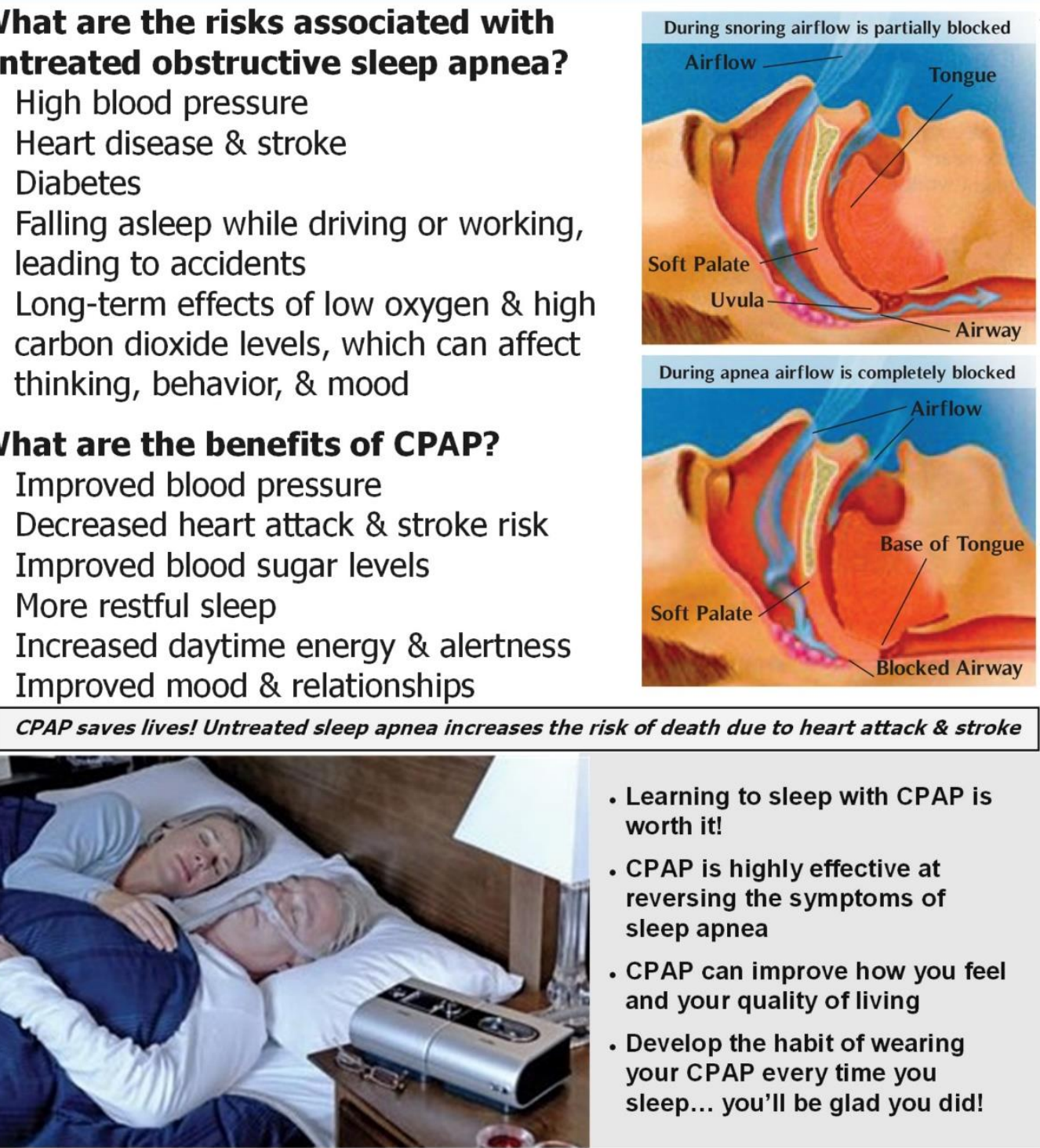

- Learning to sleep with CPAP is worth it!

- CPAP is highly effective at reversing the symptoms of sleep apnea

- CPAP can improve how you feel and your quality of living

- Develop the habit of wearing your CPAP every time you sleep... you'll be glad you did! 


\section{Appendix D}

\section{CPAP Report Card}

Participant Identification Number

Date

\begin{tabular}{|c|c|c|c|c|}
\hline & Ranges & Sleep Study & CPAP Week 1 & CPAP Month 1 \\
\hline $\begin{array}{l}\text { Severity of your obstructive sleep apnea } \\
\text { - Number of times per hour you had } \\
\text { apneas and other breathing interruptions } \\
\text { while you slept }\end{array}$ & $\begin{array}{l}\text { Normal: Under } 5 \\
\text { Mild: } 5-15 \\
\text { Moderate: Over } 15- \\
30 \\
\text { Severe: Over } 30\end{array}$ & & & \\
\hline $\begin{array}{l}\text { Setting of your CPAP machine } \\
\text { - Amount of water pressure needed to } \\
\text { keep your airway open and prevent } \\
\text { apneas while you slept }\end{array}$ & $\begin{array}{l}0 \text { to } 20 \text { centimeters } \\
\text { of water pressure }\end{array}$ & & & \\
\hline $\begin{array}{l}\text { Use of your CPAP machine } \\
\text { - Includes hours per night you wore the } \\
\text { CPAP mask } \\
\text { - Includes number of nights you wore the } \\
\text { CPAP mask }\end{array}$ & $\begin{array}{l}\text { At least four hours } \\
\text { per night for } 70 \% \\
\text { of the time ( } 5 \text { out of } \\
7 \text { nights; } 21 \text { out of } \\
30 \text { nights) }\end{array}$ & N/A & & \\
\hline $\begin{array}{l}\text { Self-evaluation of your CPAP progress } \\
\text { - What grade do you give yourself for } \\
\text { your CPAP use? }\end{array}$ & $\begin{array}{l}\text { A: Demonstrates } \\
\text { adherence } \\
\text { B: Showing steady } \\
\text { progress } \\
\text { C: Progressing, but } \\
\text { with much support } \\
\text { N: Not adherent }\end{array}$ & N/A & & \\
\hline $\begin{array}{l}\text { Provider-evaluation of your CPAP progress } \\
\text { - What grade does your respiratory } \\
\text { therapist give you for your CPAP use? }\end{array}$ & $\begin{array}{l}\text { Use same grading } \\
\text { scale as above }\end{array}$ & N/A & & \\
\hline
\end{tabular}




\section{Appendix E}

Participant ID\#

Date Entered Study

Date Exited Study

CPAP-SAVER Intervention OSA-CPAP Data Log

\begin{tabular}{|c|c|c|c|}
\hline & Sleep Study & $\begin{array}{l}\text { CPAP Week One } \\
\text { (7 Nights) }\end{array}$ & $\begin{array}{l}\text { CPAP Month One } \\
\text { (30 Nights) }\end{array}$ \\
\hline \multicolumn{4}{|l|}{ Sleep Apnea Severity } \\
\hline \multicolumn{4}{|l|}{$\begin{array}{l}\text { Apnea-Hypopnea Index } \\
\text { (Events per Hour) } \\
\text { Normal = Under } 5 \\
\text { Mild = } 5-15 \\
\text { Moderate = Over } 15-30 \\
\text { Severe }=\text { Over } 30\end{array}$} \\
\hline \multicolumn{4}{|l|}{ Oxygen Saturation Nadir (\%) } \\
\hline \multicolumn{4}{|l|}{ CPAP Settings } \\
\hline \multicolumn{4}{|l|}{ Machine Make \& Model } \\
\hline \multicolumn{4}{|l|}{ Pressure $\left(\mathrm{cm} \mathrm{H}_{2} \mathrm{O}\right)$} \\
\hline \multicolumn{4}{|l|}{ C-Flex $(1,2$, or 3$)$} \\
\hline \multicolumn{4}{|l|}{ Other Settings } \\
\hline \multicolumn{4}{|l|}{ Humidification (No or Yes) } \\
\hline \multicolumn{4}{|l|}{ Mask } \\
\hline \multicolumn{4}{|l|}{ Make \& Model } \\
\hline \multicolumn{4}{|l|}{$\begin{array}{l}\text { Type (Nasal, Pillows, or Full } \\
\text { Face) }\end{array}$} \\
\hline \multicolumn{4}{|l|}{ Size } \\
\hline \multicolumn{4}{|l|}{ CPAP Use and Adherence } \\
\hline Total CPAP Use & N/A & & \\
\hline Average Hours per Night & N/A & & \\
\hline Number Nights & N/A & & \\
\hline Meets Adherence Definition? & N/A & & \\
\hline
\end{tabular}




\section{Appendix F}

\section{CPAP-SAVER Intervention Fidelity Checklist}

Facility

RT

Date

Investigator Initials

\begin{tabular}{|c|c|c|c|c|}
\hline Fidelity Category & Fidelity Component & Yes & No & Comments/Retraining \\
\hline Target Population & $\begin{array}{l}\text { Is RT verifying that only those participants who have consented for the } \\
\text { study are receiving the intervention? }\end{array}$ & & & \\
\hline \multirow[t]{2}{*}{ Setting } & Is RT implementing the intervention in the same room each time? & & & \\
\hline & Is RT protecting privacy by closing door and taking other actions? & & & \\
\hline \multirow[t]{2}{*}{ Delivery } & Is RT using the training manual to maintain protocol? & & & \\
\hline & $\begin{array}{l}\text { Is RT ensuring that ALL participants are completing the instruments } \\
\text { before receiving the intervention or standard care? }\end{array}$ & & & \\
\hline \multirow[t]{4}{*}{ Dosage and Timing } & Is RT providing intervention only to those in the intervention group? & & & \\
\hline & $\begin{array}{l}\text { For those in the intervention group, is RT providing intervention } \\
\text { components BEFORE standard care? }\end{array}$ & & & \\
\hline & $\begin{array}{l}\text { Observe one intervention session: Is RT following the CPAP-SAVER } \\
\text { protocol for airway, video, and education, in prescribed order? } \\
\text { Airway, using model }-3 \text { minutes } \\
\text { Video }-3 \text { minutes } \\
\text { Education using OSA risks/CPAP benefits sheet }-2 \text { minutes }\end{array}$ & & & \\
\hline & $\begin{array}{l}\text { Is RT initiating report card AFTER airway, video, and education } \\
\text { components? }\end{array}$ & & & \\
\hline \multirow[t]{2}{*}{ Materials } & $\begin{array}{l}\text { Observe one intervention session: Is RT using the prescribed items to } \\
\text { implement the intervention? } \\
\text { Airway - Airway Simulator Board (Anatomy Warehouse) } \\
\text { Video - How CPAP Works (Ken Warner Remote) } \\
\text { Education - The Risks of Obstructive Sleep Apnea \& the Benefits of } \\
\text { CPAP (developed by investigator) } \\
\text { Report card - CPAP Report Card (developed by investigator) }\end{array}$ & & & \\
\hline & Does RT have ample packets/supplies to continue intervention? & & & \\
\hline RT Qualifications & $\begin{array}{l}\text { Are only those RTS who were trained to implement the intervention } \\
\text { actually implementing it? }\end{array}$ & & & \\
\hline RT Training & Does RT need a booster in training? & & & \\
\hline
\end{tabular}

Additional Comments: 


\section{Appendix G}

CPAP-SAVER Intervention Effectiveness Survey

Participant Identification Number

Date

Instructions: Please rate the following areas, based on how effective you thought they were. Circle the number that best represents your opinion, using this scale:

\begin{tabular}{|c|c|c|c|c|}
\hline Not at all & Slightly & Neutral & Somewhat & Extremely \\
\hline $\mathbf{0}$ & $\mathbf{1}$ & $\mathbf{2}$ & $\mathbf{3}$ & $\mathbf{4}$ \\
\hline
\end{tabular}

\section{Support Calls:}

Extent to which you found this information helpful

$\begin{array}{lllll}\mathbf{0} & \mathbf{1} & \mathbf{2} & \mathbf{3} & \mathbf{4} \\ \mathbf{0} & \mathbf{1} & \mathbf{2} & \mathbf{3} & 4 \\ \mathbf{0} & \mathbf{1} & \mathbf{2} & \mathbf{3} & 4 \\ \mathbf{0} & \mathbf{1} & \mathbf{2} & \mathbf{3} & 4\end{array}$

Extent to which you liked this

Extent to which you understood this

Extent to which you believe this motivated your CPAP use

Airway Model:

Extent to which you found this information helpful

Extent to which you liked this

Extent to which you understood this

Extent to which you believe this motivated your CPAP use

$\begin{array}{lllll}\mathbf{0} & \mathbf{1} & \mathbf{2} & \mathbf{3} & \mathbf{4} \\ \mathbf{0} & \mathbf{1} & \mathbf{2} & \mathbf{3} & \mathbf{4} \\ \mathbf{0} & \mathbf{1} & \mathbf{2} & \mathbf{3} & 4 \\ \mathbf{0} & \mathbf{1} & \mathbf{2} & \mathbf{3} & \mathbf{4}\end{array}$

\section{Video:}

Extent to which you found this information helpful

$\begin{array}{lllll}\mathbf{0} & \mathbf{1} & \mathbf{2} & \mathbf{3} & \mathbf{4} \\ \mathbf{0} & \mathbf{1} & \mathbf{2} & \mathbf{3} & \mathbf{4} \\ \mathbf{0} & \mathbf{1} & \mathbf{2} & \mathbf{3} & \mathbf{4} \\ \mathbf{0} & \mathbf{1} & \mathbf{2} & \mathbf{3} & 4\end{array}$

Extent to which you liked this

Extent to which you understood this

Extent to which you believe this motivated your CPAP use

\section{Education Sheet:}

Extent to which you found this information helpful

$\begin{array}{lllll}\mathbf{0} & \mathbf{1} & \mathbf{2} & \mathbf{3} & \mathbf{4} \\ \mathbf{0} & \mathbf{1} & \mathbf{2} & \mathbf{3} & \mathbf{4} \\ \mathbf{0} & \mathbf{1} & \mathbf{2} & \mathbf{3} & \mathbf{4} \\ \mathbf{0} & \mathbf{1} & \mathbf{2} & \mathbf{3} & \mathbf{4}\end{array}$

Extent to which you liked this

Extent to which you understood this

Extent to which you believe this motivated your CPAP use

\section{Report Card:}

Extent to which you found this information helpful

$\begin{array}{lllll}\mathbf{0} & \mathbf{1} & \mathbf{2} & \mathbf{3} & \mathbf{4} \\ \mathbf{0} & \mathbf{1} & \mathbf{2} & \mathbf{3} & \mathbf{4} \\ \mathbf{0} & \mathbf{1} & \mathbf{2} & \mathbf{3} & \mathbf{4} \\ \mathbf{0} & \mathbf{1} & \mathbf{2} & \mathbf{3} & \mathbf{4}\end{array}$

Extent to which you liked this

Extent to which you understood this

Extent to which you believe this motivated your CPAP use 


\section{Appendix $\mathbf{H}$}

West Virginia University School of Nursing

6700 Health Sciences South

Post Office Box 9630

Morgantown, West Virginia 26506

Date:

Dear CPAP Research Study Participant:

Upon review of the questionnaires you completed as part of this study, it was noted that your Beck Anxiety Inventory score was in the moderate to severe range. It is recommended that you contact your primary care provider to discuss this finding.

Thank you,

April L. Shapiro, MS, RN, PhD Candidate

Investigator 


\section{Appendix I}

\section{Confidentiality Agreement}

$$
\mathrm{I} \text {, }
$$
, a research assistant in the dissertation

research study being conducted by Investigator April L. Shapiro, MS, RN, PhD Candidate,

\section{Effect of the CPAP-SAVER Intervention on Adherence Among Adults with Newly Diagnosed Obstructive Sleep Apnea}

agree that I will take measures to maintain privacy and confidentiality of all participant data throughout the study. These measures include, but are not limited to,

- Not divulging the participant's participation in the study to anyone not involved in the study;

- Not divulging to the participant which study group (intervention or control) he/she is in;

- Using the participant's identification code number instead of his/her name on all studyrelated documents;

- Securing the storage of participant study-related data, both written and electronic, by using locked cabinets/doors and password-secured computers, respectively;

- Conducting study-related sessions in a private room/area; and

- Implementing other safeguards as outlined in my completed CITI training modules.

If I have any questions or concerns about confidentiality measures related to this study, I will immediately contact the investigator, April L. Shapiro, by phone at 301-707-5904 or by e-mail at ashapiro@mix.wvu.edu

This agreement is in effect upon my signing below.

Research Assistant: Print Name

Date

Research Assistant: Signature

Witness Signature 